ANNUAL YIELD AND SELECTED HYDROLOGIC DATA

FOR THE ARKANSAS RIVER BASIN COMPACT

ARKANSAS-OKLAHOMA 1992 WATER YEAR

By C.S. Barks, R.L. Blazs, and S.T. Touschner

U.S. GEOLOGICAL SURVEY

Open-File Report 93-171

Prepared in cooperation with the 


\section{U.S. DEPARTMENT OF THE INTERIOR BRUCE BABBITT, Secretary}

\section{U.S. GEOLOGICAL SURVEY}

Dallas L. Peck, Director

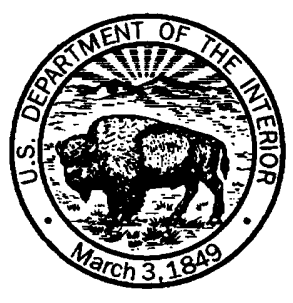

For additional information write to:

District Chief

U.S. Geological Survey 2301 Federal Office Building 700 West Capitol Avenue Little Rock, Arkansas 721201
Copies of this report can be purchased from:

U.S. Geological Survey Books and Open-File Reports Section Federal Center

Box 25425

Denver, Colorado 80225 


\section{CONTENTS}

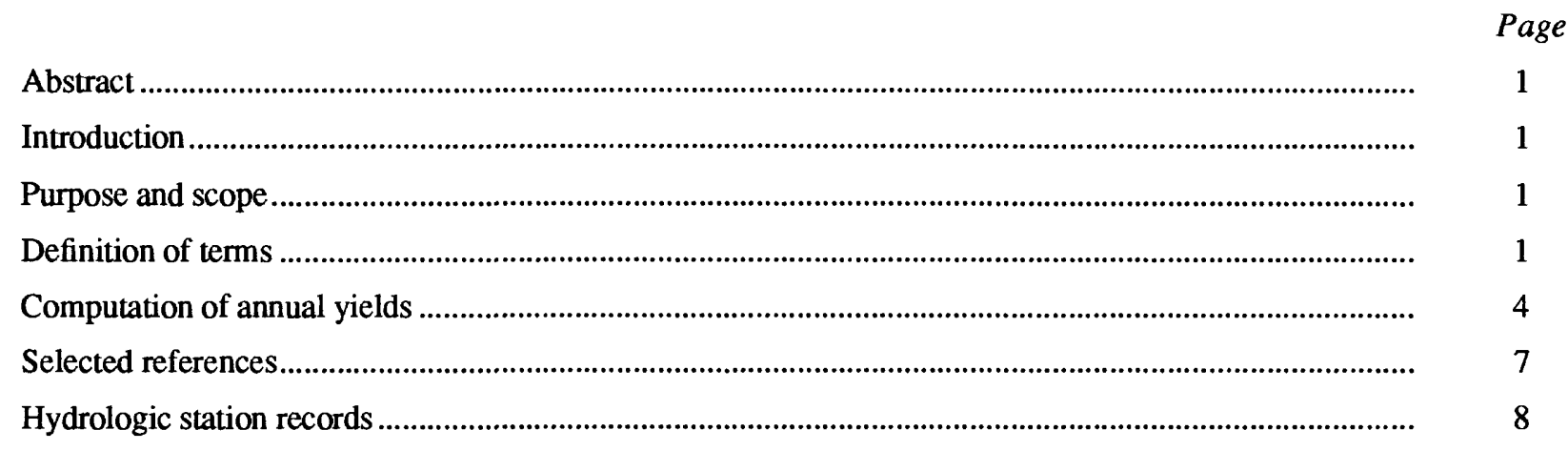

\section{ILLUSTRATIONS}

Figure 1. Map showing Arkansas-Oklahoma Arkansas River Basin Compact area and subbasins

\section{TABLES}

Table 1. Annual yield and deficiency for the subbasins for the 1992 water year, as defined in the Arkansas River Basin Compact Arkansas-Oklahoma

2. Actual runoff from the subbasins for the 1992 water year.................................................................. 5

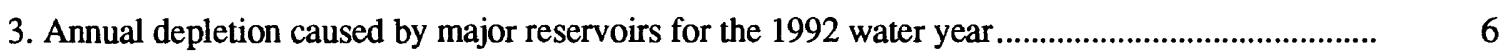

\section{CONVERSION FACTORS}

\section{Multiply}

inch (in)

foot (ft)

mile (mi)

acre

square mile $\left(\mathrm{mi}^{2}\right)$

cubic foot $\left(\mathrm{ft}^{3}\right)$

acre-foot (acre-ft)

cubic foot per second $\left(\mathrm{ft}^{3} / \mathrm{s}\right)$

ton per day (ton/d)
By

25.4

0.3048

1.609

4,047

0.004047

2.590

0.02832

1,233

$1.233 \times 10^{-6}$

28.32

0.02832

0.9072
To obtain

millimeter

meter

kilometer

square meter square kilometer

square kilometer

cubic meter

cubic meter cubic kilometer

liter per second cubic meter per second megagram per day

Temperature in degrees Celsius $\left({ }^{\circ} \mathrm{C}\right)$ can be converted to degrees Fahrenheit $\left({ }^{\circ} \mathrm{F}\right)$ as follows:

$$
{ }^{\circ} \mathrm{F}=1.8 \times{ }^{\circ} \mathrm{C}+32
$$




\title{
ANNUAL YIELD AND SELECTED HYDROLOGIC DATA FOR THE ARKANSAS RIVER BASIN COMPACT ARKANSAS-OKLAHOMA 1992 WATER YEAR
}

\author{
By C.S. Barks, R.L. Blazs, and S.T. Touschner
}

\begin{abstract}
The computed annual yield and deficiency of the subbasins as defined in the Arkansas River Basin Compact, Arkansas-Oklahoma, are given in tables for the 1992 water year. Actual runoff from the subbasins and depletion caused by major reservoirs in the compact area also are given in tabular form. Monthly maximum, minimum, and mean discharges are shown for the 14 streamflow stations used in computing annual yield. Water-quality data are shown for 14 water-quality stations sampled in the Arkansas River Basin.
\end{abstract}

\section{INTRODUCTION}

In 1955, the Congress of the United States granted consent to Arkansas and Oklahoma to enter into a compact for the apportionment of the waters of the Arkansas River and its tributaries as they affect the two states. An ArkansasOklahoma Arkansas River Compact committee was created with a Federal Representative acting as chairman. After research and deliberate negotiations had been completed, both states approved the Arkansas River Basin Compact, Arkansas-Oklahoma, 1972. To meet the requirements of the Compact, stateline yields of the Arkansas River Basin are determined at the end of each year.

This report was prepared by the U.S. Geological Survey in cooperation with the Arkansas River Basin Compact, Arkansas-Oklahoma, Commission. Stream- flow data and water-quality data were furnished by the U.S. Geological Survey. The U.S. Army Corps of Engineers, Tulsa District furnished data from the Webbers Falls, Tenkiller Ferry, Robert S. Kerr, Wister, and Fort Gibson Lakes.

\section{PURPOSE AND SCOPE}

The purpose of this report is to present the annual yields and deficiencies computed for the 1992 water year for subbasins in the Arkansas River Basin as defined in the Arkansas River Basin Compact, Arkansas-Oklahoma, 1972. The report includes data from 14 streamflow stations and 14 water-quality stations sampled in the Arkansas River Basin during the 1992 water year. The area included in the Compact is shown on figure 1.

\section{DEFINITION OF TERMS}

The following terms used in this report are taken from Article II of the Arkansas River Basin Compact, ArkansasOklahoma, 1972.

The term "Arkansas River Basin" means all of the drainage basin of the Arkansas River and its tributaries from a point immediately downstream from the confluence of the Neosho River with the Arkansas River (fig. 1) to a point immediately downstream from the confluence of Lee Creek with the Arkansas River, together with the drainage basin of Spavinaw Creek in Arkansas (fig. 1), but excludes that part of the drainage basin of the Canadian River upstream from Lake Eufaula Dam.

The term "Spavinaw Creek Subbasin" means the drainage area of Spavinaw Creek in the State of Arkansas.

The term "Illinois River Subbasin" means the drainage area of the Illinois River in the State of Arkansas.

The term "Lee Creek Subbasin" means the drainage area of Lee Creek in the State of Arkansas and in the State of Oklahoma.

The term "Poteau River Subbasin" means the drainage area of the Poteau River in the State of Arkansas.

The term "Arkansas River Subbasin" means all areas of the Arkansas River Basin except the four subbasins described previously. 


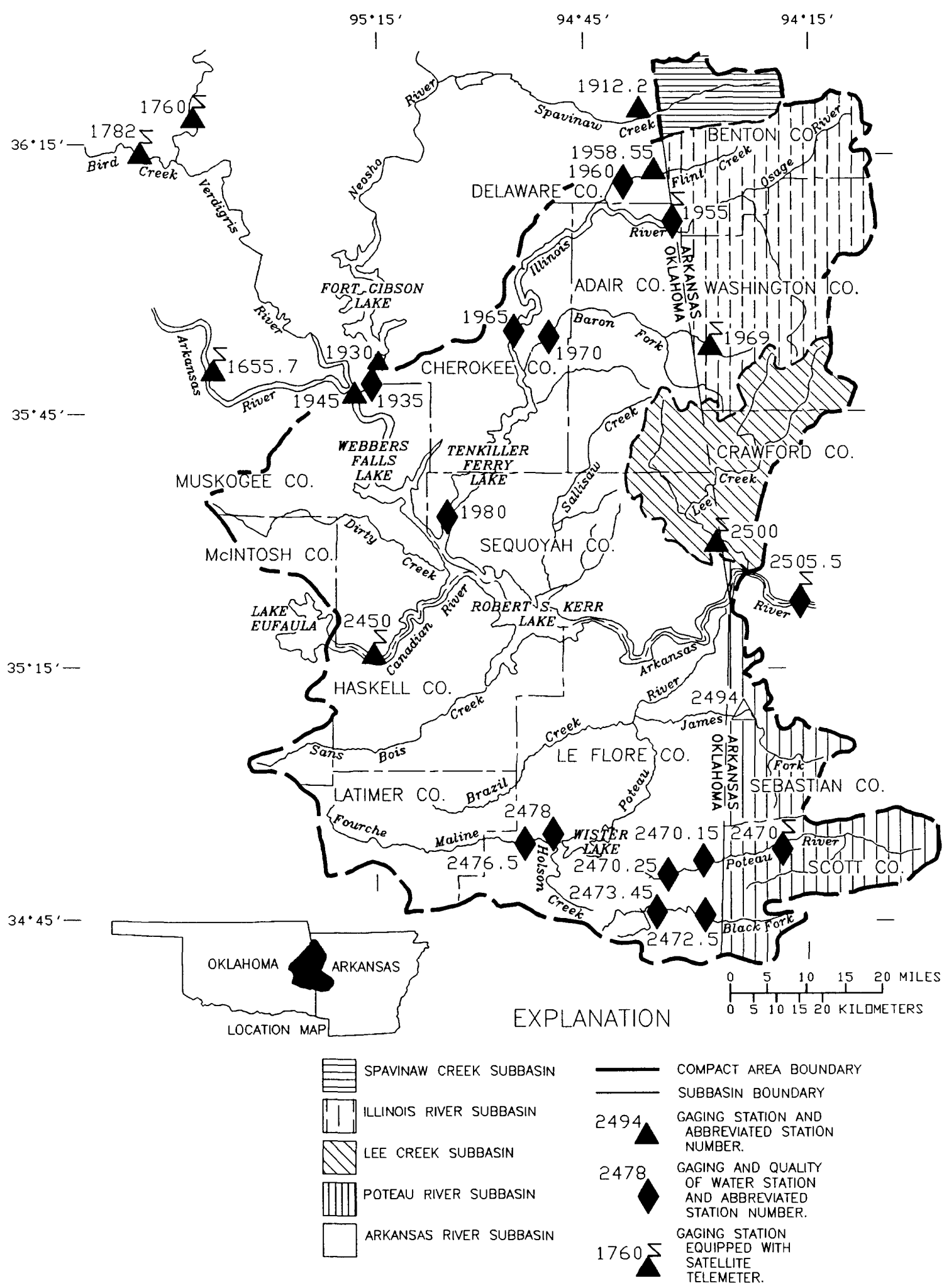

Figure 1.--Arkansas-Oklahoma Arkansas River Basin Compact area and subbasins. 
The term "water year" means a 12-month period beginning on October 1 and ending September 30.

The term "annual yield" means the computed annual gross runoff from any specified subbasin. The runoff would have passed any certain point on a stream and would have originated within any specified area under natural conditions, without any manmade depletion or accretion during the water year.

Terms related to streamflow, water-quality, and other hydrologic data, as used in this report, are defined below.

Acre-foot is the quantity of water required to cover 1 acre to a depth of 1 foot and is equivalent to 43,560 cubic feet.

Bacteria are microscopic unicellular organisms, typically spherical, rodlike, or spiral and threadlike in shape, often clumped into colonies. Some bacteria cause disease, others perform an essential role in nature in the recycling of materials; for example, by decomposing organic matter into a form available for reuse by plants.

Fecal coliform bacteria are present in the intestines or feces of warm-blooded animals. They are often used as indicators of the sanitary quality of the water. In the laboratory, they are defined as all the organisms that produce blue colonies within 24 hours when incubated at $44.5^{\circ} \mathrm{C} \pm 0.2^{\circ} \mathrm{C}$ on $\mathrm{M}-\mathrm{FC}$ medium (nutrient medium for bacterial growth). Their concentrations are expressed as number of colonies per 100 milliliters $(\mathrm{mL})$ of sample.

Fecal streptococcal bacteria also are present in intestines of warm-blooded animals. Their presence in water is considered to verify fecal pollution. They are characterized as grampositive, cocci bacteria that are capable of growth in brain-heart infusion broth. These bacteria also are defined as all the organisms that produce red or pink colonies within 48 hours at $35^{\circ} \mathrm{C} \pm 0.5^{\circ} \mathrm{C}$ on $\mathrm{KF}$-streptococcus agar (nutrient medium for bacterial growth). Their concentrations are expressed as number of colonies per $100 \mathrm{~mL}$ of sample.

Code numbers have been assigned for agencies collecting and analyzing samples, and are listed in waterquality tables of this report as follows:

1028 Oklahoma District, WRD, U.S. Geological Survey

80513 Arkansas District, WRD, U.S. Geological Survey

80020 National Water Quality Laboratory, WRD, U.S. Geological Survey.

Contents are the volume of water in a reservoir or lake. Unless other-wise indicated, volume is computed on the basis of a level pool and does not include bank storage.

Cubic foot per second is the rate of discharge representing a volume of 1 cubic foot passing a specified point during 1 second.

Discharge is the volume of water that passes a given point within a given period of time.

Instantaneous discharge is the discharge at a particular instant of time.

Mean discharge (Mean) is the arithmetic average of individual daily mean discharges during a specific period.

Dissolved refers to the material in a representative water sample that passes through a 0.45 -micrometer membrane filter. This is a convenient operational definition used by Federal agencies that collect water data. Determinations of "dissolved" constituents are made on subsamples of the filtrate.

Dissolved oxygen content of water in equilibrium with air is a function of atmospheric pressure and temperature and the dissolved-solids concentration of the water. The ability of water to retain oxygen decreases with increasing temperature or dissolved solids, with small temperature changes having the more significant effect. Photosynthesis and respiration may cause diurnal variations in dissolved-oxygen concentration in water of some streams.

Drainage area of a stream at a specified location is that area, measured in a horizontal plane, enclosed by a topographic divide from which direct surface runoff from precipitation normally drains by gravity into the stream upstream from the specified point. Figures of drainage area given herein include all closed basins, or noncontributing areas within the area, unless otherwise noted.

Gaging station is a particular site on a stream, canal, lake, or reservoir where systematic observations of hydrologic data are obtained. 
Hardness of water is a physical-chemical characteristic that is commonly recognized by the increased quantity of soap required to produce lather. It is attributable to the presence of alkaline earths (principally calcium and magnesium) and is expressed as equivalent calcium carbonate $\left(\mathrm{CaCO}_{3}\right)$.

Sediment is solid material that originates mostly from disintegrated rocks and is transported by, suspended in, or deposited from water; it includes chemical and biochemical precipitates and decomposed organic material, such as humus. The quantity, characteristics, and cause of the occurrence of sediment in streams are influenced by environmental factors. Some major factors are degree of slope, length of slope, soil characteristics, land usage, and quantity and intensity of precipitation.

Mean concentration is the time-weighted concentration of suspended sediment passing a stream cross section during a 24-hour period.

Suspended sediment is the sediment that at any given time is maintained in suspension by the upward components of turbulent currents or that exists in suspension as a colloid.

Suspended-sediment concentration is the velocity-weighted concentration of suspended sediment in the sampled zone (from the water surface to a point approximately $0.3 \mathrm{ft}$ above the bed), expressed as milligrams of dry sediment per liter of water-sediment mixture $(\mathrm{mg} / \mathrm{L})$.

Suspended-sediment discharge (tons/day) is the rate at which dry weight of sediment passes a section of a stream or is the quantity of sediment, as measured by dry weight or volume, that passes a section in a given time. It is computed by multiplying discharge by milligrams per liter by 0.0027 .

Sodium-absorption-ratio (SAR) is the expression of relative activity of sodium ions in exchange reactions with soil and is an index of sodium or alkali hazard to the soil. Water varies, in respect to sodium hazard, from that which can be used for irrigation on almost all soils to that which generally is unsatisfactory for irrigation.

Specific conductance is a measure of the ability of water to conduct an electrical current. It is expressed in microsiemens per centimeter at $25^{\circ} \mathrm{C}$. Specific conductance is related to the type and concentration of ions in solution and can be used for approximating the dissolved-solids concentration of the water. Commonly, the concentration of dissolved solids (in milligrams per liter) is about 65 percent of the specific conductance (in microsiemens). This relation is not constant from stream to stream, and it may vary in the same source with changes in the composition of the water.

Stage-discharge relation is the relation between gage height (stage) and the volume of water, per unit of time, flowing past the gage in a channel.

Streamflow is the discharge that occurs in a natural channel. Although the term "discharge" can be applied to the flow of a canal, the word "streamflow" uniquely describes the discharge in a surface stream course. The term "streamflow" is more general than "runoff", as streamflow may be applied to discharge whether or not it is affected by diversion or regulation.

Tons per day is the quantity of substance in solution or suspension that passes a stream section during a 24-hour period.

Total is the total amount of a given constituent in a representative water-suspended sediment sample, regardless of the constituent's physical or chemical form. This term is used only when the analytical procedure assures measurement of at least 95 percent of the constituent present in both the dissolved and suspended phases of the sample. A knowledge of the expected form of the constituent in the sample, as well as the analytical methodology used, is required to judge when the results should be reported as "total". (Note that the word "total" does double duty here, indicating that the sample consists of a water-suspended-sediment mixture and that the analytical method determines all of the constituent in the sample.)

\section{COMPUTATION OF ANNUAL YIELDS}

The annual yield and deficiency (table 1) for each subbasin were computed as described in Appendix I to the Arkansas River Basin Compact Arkansas-Oklahoma, 1972, supplement No. 1. Actual runoff for the subbasins (table 2) was computed as described in the Compact except for the stations Arkansas River at Muskogee, which has been discontinued, and Arkansas River at Van Buren, which has been moved 7.9 miles downstream. 


\section{Table 1. -Annual yield and deficiency for the subbasins for the 1992 water year, as defined In the Arkansas River Basin Compact Arkansas-Oklahoma}

[Flow in cubic feet per second]

\begin{tabular}{|c|c|c|c|c|c|c|}
\hline Subbasin & $\begin{array}{l}\text { Actual runoff } \\
\text { from the } \\
\text { subbasins }\end{array}$ & $\begin{array}{c}\text { Total } \\
\text { depletions or } \\
\text { accretions (-) }\end{array}$ & Annual yield & $\begin{array}{l}{ }^{\text {apercent }} \\
\text { depletion } \\
\text { allowed }\end{array}$ & $\begin{array}{l}\text { Minimum } \\
\text { required flow }\end{array}$ & ${ }^{b}$ Deficiency \\
\hline Spavinaw Creek & 96.4 & ${ }^{c} 0.4$ & 96.8 & 50 & 48.4 & 0 \\
\hline Illinois River & 791 & $c_{573}$ & 1,364 & 60 & 546 & 0 \\
\hline Lee Creek & 623 & ${ }^{c} 0.2$ & 623 & 100 & 0 & 0 \\
\hline Poteau River & 653 & $c_{2.8}$ & 656 & 60 & 262 & 0 \\
\hline Arkansas River & 7,418 & 198 & 7,616 & 60 & 3,046 & 0 \\
\hline
\end{tabular}

${ }^{a}$ Defined in the Arkansas River Basin Compact, Arkansas-Oklahoma, 1972.

${ }^{b}$ The amount the actual runoff is less than the minimum required flow.

${ }^{\mathrm{c}}$ Based on 1991 water year water-use data..

Table 2. --Actual runoff from the subbasins for the 1992 water year

[In cubic feet per second; D.A. = drainage area]

\begin{tabular}{|c|c|c|c|c|c|}
\hline Month & $\begin{array}{l}{ }^{\text {a} S p a v i n a w ~ C r e e k ~} \\
\text { D.A. }=135 \mathrm{mi}^{2}\end{array}$ & $\begin{array}{l}\text { bIllinois River } \\
\text { D.A. }=744 \mathrm{mi}^{2}\end{array}$ & $\begin{array}{c}{ }^{c} \text { Lee Creek } \\
\text { D.A. }=464 \mathrm{mi}^{2}\end{array}$ & $\begin{array}{l}{ }^{d} \text { Poteau River } \\
\text { D.A. }=536 \mathrm{mi}^{2}\end{array}$ & $\begin{array}{l}{ }^{\mathrm{e}} \text { Arkansas River } \\
\text { D.A., }=4,553 \mathrm{mi}^{2}\end{array}$ \\
\hline October & 19.7 & 543 & 561 & 1,120 & 5,943 \\
\hline November & 123 & 1,570 & 1,280 & 1,450 & 14,706 \\
\hline December & 221 & 1,780 & 1,400 & 2,260 & 14,785 \\
\hline January & 67.9 & 625 & 496 & 660 & 6,795 \\
\hline February & 116 & 612 & 400 & 321 & 3,374 \\
\hline March & 58.9 & 366 & 473 & 327 & 3,360 \\
\hline April & 104 & 435 & 478 & 148 & 5,371 \\
\hline May & 73.8 & 567 & 463 & 125 & 6,442 \\
\hline June & 247 & 1,360 & 1,140 & 991 & 13,657 \\
\hline July & 50.5 & 533 & 336 & 110 & 4,544 \\
\hline August & 47.9 & 792 & 350 & 17.6 & 3,361 \\
\hline September & 32.8 & 319 & 98.8 & 293 & 6,763 \\
\hline 1992 water year & 96.4 & 791 & 623 & 653 & 7,418 \\
\hline 1992 water year (acre-ft) & 69,800 & 573,000 & 451,000 & 473,000 & $5,374,000$ \\
\hline
\end{tabular}

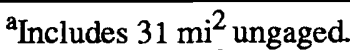

b Includes $63 \mathrm{mi}^{2}$ ungaged.

'Includes $38 \mathrm{mi}^{2}$ ungaged.

${ }^{\mathrm{d}}$ Includes $125 \mathrm{mi}^{2}$ ungaged.

${ }^{\text {e}}$ Computed by subtracting drainage area at Arkansas River at Muskogee, Canadian River near Whitefield, Illinois River Subbasin, Lee Creek Subbasin, and Poteau River Subbasin from drainage area at Arkansas River at James W. Trimble Dam, near Van Buren, Arkansas.

Annual depletion caused by major reservoirs (table 3) was computed for the four major reservoirs in the basin as described in Appendix I to the Compact. Depletions caused by small reservoirs and minor diversions for municipal and agricultural use are considered by subbasins in table 1. 
A compilation of the areas and capacities of lakes and ponds in Arkansas, updated in 1981, conducted by the Arkansas Soil and Water Conservation Commission was used to evaluate depletions caused by small reservoirs in the Poteau River, Lee Creek, Spavinaw Creek, and Illinois River subbasins. Analysis indicated that their impact on the depletions in any subbasin, except Illinois River, was probably insignificant. Information on depletions continue to be gathered in order to reevaluate their present impact.

Streamflow data used in the computations are given in hydrologic station records (p. 8 to 42). The station description under "Remarks" states the degree of accuracy of the records. "Excellent" means that about 95 percent of the daily discharges are within 5 percent of the actual discharge, "good" means within 10 percent, and "fair" means within 15 percent. "Poor" means that daily discharges have less than "fair" accuracy.

Table 3. --Annual depletion caused by major reservoirs for the 1992 water year

\begin{tabular}{|c|c|c|c|c|c|c|c|}
\hline Reservoir & $\begin{array}{l}\text { Year-end } \\
\text { contents } \\
\text { (acre-ft) }\end{array}$ & $\begin{array}{c}\text { Change in } \\
\text { contents in } \\
\text { water year } \\
\text { (acre-ft) }\end{array}$ & $\begin{array}{l}\text { aPrecipitation } \\
\text { on reservoir } \\
\text { surface } \\
\text { (inches) }\end{array}$ & $\begin{array}{c}\text { bevaporation } \\
\text { from } \\
\text { reservoir } \\
\text { (inches) }\end{array}$ & $\begin{array}{l}{ }^{\text {'Diversions }} \\
\text { (acre-ft) }\end{array}$ & $\begin{array}{l}\text { Depletion } \\
\text { (acre-ft) }\end{array}$ & $\begin{array}{l}\text { Depletion } \\
\left(\mathrm{ft}^{3} / \mathrm{s}\right)\end{array}$ \\
\hline Webbers Falls & 157,600 & $-4,000$ & 56.85 & 47.47 & 0 & $+9,100$ & +12.6 \\
\hline Tenkiller Ferry & 647,600 & $+18,300$ & 59.23 & 35.49 & 6,220 & $+27,000$ & +37.3 \\
\hline Robert S. Kerr & 524,800 & $+7,700$ & 48.22 & 43.79 & 0 & $+48,100$ & +66.4 \\
\hline Wister & 102,000 & $+52,280$ & 65.25 & 34.61 & 6,154 & $+59,350$ & +81.9 \\
\hline
\end{tabular}

${ }^{\mathrm{a}}$ From U.S. Corps of Engineers, Tulsa District.

${ }^{b}$ Adjusted for pan coefficient of 0.70 (from Wisler and Brater, 1949). 


\section{SELECTED REFERENCES}

Arkansas River Compact Committee, 1972, Arkansas River Basin Compact Arkansas-Oklahoma, 1972, with Supplemental Interpretive Comments, Supplement No. 1: Austin, Texas, 31 p.

Arkansas Soil and Water Conservation Commission, 1981, Arkansas State Water Plan - Lakes of Arkansas, 157 p.

Wisler, C.D., and Brater, E.F., 1949, Hydrology: New York, John Wiley \& Sons, Inc., 150 p. 
HYDROLOGIC STATION RECORDS 


\section{STREAMFLOW}

07165570 Arkansas River near Haskell, Oklahoma

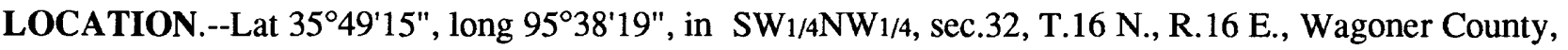
near left downstream abutment of old bridge downstream from State Highway 104, 2.0 mi east of Haskell, 23.5 mi upstream from Verdigris River, and at mile 483.7.

DRAINAGE AREA.--75,473 $\mathrm{mi}^{2}$, of which $12,541 \mathrm{mi}^{2}$ probably is noncontributing.

AVERAGE DISCHARGE.--20 years, $9,387 \mathrm{ft}^{3} / \mathrm{s}$.

EXTREMES.--June 1972 to current year: Maximum discharge, $259,000 \mathrm{ft}^{3} / \mathrm{s}$ Oct. 6, 1986; minimum daily, $87 \mathrm{ft}^{3} / \mathrm{s}$ Sept. 13, 1988.

REMARKS.--Records fair, except for $858 \mathrm{mi}^{2}$ intervening area. Flow regulated by Keystone Lake, $55.1 \mathrm{mi}$ upstream. Satellite telemeter at station.

Monthly and yearly discharge

\begin{tabular}{lccccc}
\hline Month & $\begin{array}{c}\text { Total } \\
\left(\mathrm{ft}^{3} / \mathrm{s}\right)\end{array}$ & $\begin{array}{c}\text { Maximum } \\
\text { daily } \\
\left(\mathrm{ft}^{3} / \mathrm{s}\right)\end{array}$ & $\begin{array}{c}\text { Minimum } \\
\text { daily } \\
\left(\mathrm{ft}^{3} / \mathrm{s}\right)\end{array}$ & $\begin{array}{c}\text { Mean } \\
\left(\mathrm{ft}^{3} / \mathrm{s}\right)\end{array}$ & $\begin{array}{c}\text { Runoff in } \\
\text { acre-feet }\end{array}$ \\
\hline October & 74,943 & 6,290 & 953 & 2,418 & 148,600 \\
November & 131,170 & 8,720 & 1,520 & 4,372 & 260,200 \\
December & 267,660 & 23,000 & 1,080 & 8,634 & 530,900 \\
January & 216,410 & 14,100 & 1,050 & 6,981 & 429,200 \\
February & 100,710 & 6,470 & 1,100 & 3,473 & 199,800 \\
March & 92,030 & 6,660 & 1,300 & 2,969 & 182,500 \\
April & 130,360 & 8,620 & 1,200 & 4,345 & 258,600 \\
May & 126,220 & 6,860 & 1,560 & 4,072 & 250,400 \\
June & 443,160 & 25,500 & 1,340 & 14,770 & 879,000 \\
July & 417,410 & 22,700 & 6,340 & 13,460 & 827,900 \\
August & 519,140 & 25,600 & 6,010 & 16,750 & $1,030,000$ \\
September & 265,630 & 13,900 & 1,950 & 8,854 & 526,900 \\
& & & & & 7,609 \\
\hline Water Year 1992 & $2,784,840$ & 25,600 & 953 & $5,524,000$ \\
\hline
\end{tabular}




\section{STREAMFLOW}

07176000 Verdigris River near Claremore, Oklahoma

LOCATION.--Lat $36^{\circ} 18^{\prime} 26^{\prime \prime}$, long 95 $41^{\prime} 52^{\prime \prime}$, in NE1/4NW1/4, sec.15, T.21 N., R.15 E., Rogers County, on left bank on downstream side of bridge on State Highway 20,2.3 mi downstream from Caney River, 4.5 mi west of Claremore, 12.4 mi upstream from Bird Creek, and at mile 76.0.

DRAINAGE AREA.--6,534 $\mathrm{mi}^{2}$.

AVERAGE DISCHARGE.--27 years (water years 1936-62), 3,723 ft 3 s; 28 years (water years 1965-92), $4,301 \mathrm{ft}^{3} / \mathrm{s}$.

EXTREMES.--October 1935 to current year: Maximum discharge, 182,000 $\mathrm{ft}^{3} / \mathrm{s}$ May 21, 1943; no flow at times in 1936, 1939-40, 1956.

REMARKS.--Records fair. Flow regulated since May 1963 by Oologah Lake 14.3 mi upstream; some regulation by dams in Kansas since 1949 and by Hulah Lake since 1950. Satellite telemeter at station.

Monthly and yearly discharge

\begin{tabular}{lcccrc}
\hline Month & $\begin{array}{c}\text { Total } \\
\left(\mathrm{ft}^{3} / \mathrm{s}\right)\end{array}$ & $\begin{array}{c}\text { Maximum } \\
\text { daily } \\
\left(\mathrm{ft}^{3} / \mathrm{s}\right)\end{array}$ & $\begin{array}{c}\text { Minimum } \\
\text { daily } \\
\left(\mathrm{ft}^{3} / \mathrm{s}\right)\end{array}$ & $\begin{array}{c}\text { Mean } \\
\left(\mathrm{ft}^{3} / \mathrm{s}\right)\end{array}$ & $\begin{array}{r}\text { Runoff in } \\
\text { acre-feet }\end{array}$ \\
\hline October & 4,913 & 1,050 & 35 & 158 & 9,740 \\
November & 12,998 & 3,260 & 51 & 433 & 25,780 \\
December & 74,773 & 11,600 & 120 & 2,412 & 148,300 \\
January & 82,112 & 6,530 & 310 & 2,649 & 162,900 \\
February & 83,241 & 9,290 & 204 & 2,870 & 165,100 \\
March & 70,296 & 4,430 & 369 & 2,268 & 139,400 \\
April & 83,497 & 9,510 & 350 & 2,783 & 165,600 \\
May & 25,494 & 1,560 & 228 & 822 & 50,570 \\
June & 190,005 & 13,400 & 515 & 6,333 & 376,900 \\
July & 239,220 & 15,000 & 2,280 & 7,717 & 474,500 \\
August & 218,640 & 16,600 & 2,130 & 7,053 & 433,700 \\
September & 43,322 & 11,000 & 170 & 1,444 & 85,930 \\
& & & & & $2,238,000$ \\
\hline Water Year 1992 & $1,128,521$ & 16,600 & 35 & 3,083 & \\
\hline & & & & & \\
\hline
\end{tabular}




\section{STREAMFLOW}

07178200 Bird Creek at State Highway 266 near Catoosa, Oklahoma

LOCATION.--Lat $36^{\circ} 13^{\prime} 23^{\prime \prime}$, long 95\%49'09", in SE1/4SE1/4, sec.9, T.20 N., R.14 E., Tulsa County,, near left on downstream abutment of bridge, $2.3 \mathrm{mi}$ downstream from Elm Creek, 5 mi northwest of Catoosa High School, and at mile 9.5.

DRAINAGE AREA.--1,103 $\mathrm{mi}^{2}$.

AVERAGE DISCHARGE.--4 years, $856 \mathrm{ft}^{3} / \mathrm{s}$.

EXTREMES.--August 1988 to current year: Maximum discharge, 18,500 f $\mathrm{ft}^{3} / \mathrm{s} \mathrm{Mar.} \mathrm{14,} \mathrm{1990,} \mathrm{gage} \mathrm{height,}$ $28.39 \mathrm{ft}$; minimum daily discharge, $81 \mathrm{ft}^{3} / \mathrm{s}$, Oct. 20,1991

REMARKS.--Records good. Some regulation by Skiatook Lake (station 07177400). Satellite telemeter at station.

Monthly and yearly discharge

\begin{tabular}{lccccc}
\hline Month & $\begin{array}{c}\text { Total } \\
\left(\mathrm{ft}^{3} / \mathrm{s}\right)\end{array}$ & $\begin{array}{c}\text { Maximum } \\
\text { daily } \\
\left(\mathrm{ft}^{3} / \mathrm{s}\right)\end{array}$ & $\begin{array}{c}\text { Minimum } \\
\text { daily } \\
\left(\mathrm{ft}^{3} / \mathrm{s}\right)\end{array}$ & $\begin{array}{c}\text { Mean } \\
\left(\mathrm{ft}^{3} / \mathrm{s}\right)\end{array}$ & $\begin{array}{r}\text { Runoff in } \\
\text { acre-feet }\end{array}$ \\
\hline October & 12,834 & 3,220 & 81 & 414 & 25,460 \\
November & 19,470 & 7,460 & 142 & 649 & 38,620 \\
December & 51,067 & 13,100 & 176 & 1,647 & 101,300 \\
January & 13,908 & 1,950 & 211 & 449 & 27,590 \\
February & 25,961 & 5,200 & 152 & 895 & 51,490 \\
March & 7,921 & 757 & 128 & 256 & 15,710 \\
April & 22,299 & 2,640 & 223 & 743 & 44,230 \\
May & 16,317 & 2,680 & 231 & 526 & 32,360 \\
June & 100,663 & 9,070 & 513 & 3,355 & 199,700 \\
July & 20,114 & 3,500 & 249 & 649 & 39,900 \\
August & 10,533 & 1,310 & 162 & 340 & 20,890 \\
September & 6,640 & 568 & 168 & 221 & 13,170 \\
& & & & & 841 \\
\hline Water Year 1992 & 307,727 & 13,100 & 81 & & 610,400 \\
\hline
\end{tabular}




\section{STREAMFLOW}

\section{Spavinaw Creek near Sycamore, Oklahoma}

LOCATION.--Lat $36^{\circ} 20^{\prime} 07^{\prime \prime}$, long 94 $38^{\prime} 27^{\prime \prime}$, in NE1/4NW1/4, sec.4, T.21 N., R.25 E., Delaware County, on right bank $1.8 \mathrm{mi}$ upstream from Cherokee Creek, $4.8 \mathrm{mi}$ northeast of Row, $6.5 \mathrm{mi}$ southeast of Sycamore, and at mile 35.0.

DRAINAGE AREA.-- $133 \mathrm{mi}^{2}$

AVERAGE DISCHARGE.--31 years, $109 \mathrm{ft}^{3} / \mathrm{s}$.

EXTREMES.--October 1961 to current year: Maximum discharge, 39,800 $\mathrm{ft}^{3} / \mathrm{s} \quad$ July 27, 1975; minimum, $1.2 \mathrm{ft}^{3} / \mathrm{s}$ Aug. 9, 1964.

REMARKS.--Records good. Satellite telemeter at station.

Monthly and yearly discharge

\begin{tabular}{lccccc}
\hline & $\begin{array}{c}\text { Total } \\
\left(\mathrm{ft}^{3} / \mathrm{s}\right)\end{array}$ & $\begin{array}{c}\text { Maximum } \\
\text { daily } \\
\left(\mathrm{ft}^{3} / \mathrm{s}\right)\end{array}$ & $\begin{array}{c}\text { Minimum } \\
\text { daily } \\
\left(\mathrm{ft}^{3} / \mathrm{s}\right)\end{array}$ & $\begin{array}{c}\text { Mean } \\
\left(\mathrm{ft}^{3} / \mathrm{s}\right)\end{array}$ & $\begin{array}{c}\text { Runoff in } \\
\text { acre-feet }\end{array}$ \\
\hline October & 601 & 44 & 15 & 19.4 & 1,190 \\
November & 3,627 & 422 & 40 & 121 & 7,190 \\
December & 6,751 & 1,210 & 71 & 218 & 13,390 \\
January & 2,075 & 108 & 49 & 66.9 & 4,120 \\
February & 3,305 & 307 & 45 & 114 & 6,560 \\
March & 1,798 & 73 & 47 & 58.0 & 3,570 \\
April & 3,090 & 438 & 41 & 103 & 6,130 \\
May & 2,255 & 137 & 48 & 72.7 & 4,470 \\
June & 7,279 & 930 & 63 & 243 & 14,440 \\
July & 1,543 & 71 & 39 & 49.8 & 3,060 \\
August & 1,464 & 79 & 30 & 47.2 & 2,900 \\
September & 969 & 45 & 27 & 32.3 & 1,920 \\
& & & 15 & 95.0 & 68,940 \\
\hline Water Year 1992 & 34,757 & 1,210 & & & \\
\hline
\end{tabular}




\section{STREAMFLOW}

07193000 Fort Gibson Lake near Fort Gibson, Oklahoma

LOCATION.--Lat $35^{\circ} 51^{\prime} 15^{\prime \prime}$, long 95 $13^{\circ} 45^{\prime \prime}$, in sec.19, T.16 N., R. 19 E., Cherokee County, at Fort Gibson Dam, 5 mi north of Fort Gibson, and at mile 7.7.

DRAINAGE AREA.--12,492 $\mathrm{mi}^{2}$.

REMARKS.--Flow furnished by U.S. Army Corps of Engineers, Tulsa District.

Monthly and yearly discharge

\begin{tabular}{lrrr}
\hline Month & $\begin{array}{l}\text { Total } \\
\left(\mathrm{ft}^{3} / \mathrm{s}\right)\end{array}$ & $\begin{array}{c}\text { Mean } \\
\left(\mathrm{ft}^{3} / \mathrm{s}\right)\end{array}$ & $\begin{array}{c}\text { Runoff in } \\
\text { acre-feet }\end{array}$ \\
\hline October & 15,639 & 504 & 31,000 \\
November & 102,606 & 3,420 & 203,500 \\
December & 264,496 & 8,532 & 524,600 \\
January & 139,490 & 4,499 & 276,600 \\
February & 180,554 & 6,226 & 358,100 \\
March & 151,640 & 4,891 & 300,700 \\
April & 214,233 & 7,141 & 424,900 \\
May & 45,944 & 1,482 & 91,120 \\
June & 432,392 & 14,413 & 857,640 \\
July & 743,625 & 23,987 & $1,474,900$ \\
August & 422,409 & 13,626 & 837,840 \\
September & 184,607 & 6,153 & 366,100 \\
\hline Water Year 1992 & & & $5,747,000$ \\
\hline
\end{tabular}




\section{ARKANSAS RIVER BASIN}

\section{NEOSHO RIVER BELOW FORT GIBSON LAKE NEAR FORT GIBSON, OKLAHOMA}

(National stream-quality accounting network station)

LOCATION . --Lat 35'51'10", long 95¹3'44", in NW1/4NW1/4, sec.19, T.16 N., R.20 E., Cherokee County, Hydrologic Unit 11070209, on left bank $1.1 \mathrm{mi}$ downstream from Fort Gibson Dam, 3.5 mi north of Fort Gibson, and at mile 6.6.

DRATNAGE AREA--12,495 $\mathrm{mi}^{2}$.

PERIOD OF RECORD.--Water years 1952 to current year.

PERIOD OF DAILY RECORD.--

SPECIFIC CONDUCTANCE : October 1951 to September 1963, October 1973 to January 1982.

WATER TEMPERATURE: October 1951 to September 1963, October 1973 to January 1982.

REMARKS.--Samples were collected bimonthly and specific conductance, $\mathrm{pH}$, water temperature, dissolved oxygen, and alkalinity were determined in the field.

\section{WATER-QUALITY DATA, WATER YEAR OCTOBER 1991 TO SEPTEMBER 1992}

[Five-digit numbers in parentheses are STORET parameter codes used for computer storage of data; $\mathrm{K}$, non-ideal count; US/CM = microsiemens per centimeter at 25 degrees Celsius; NTU = nephelometric turbitidy units; MG/L = milligrams per liter; MM = millimeters; UM$\mathrm{MF}=$ micrometer membrane filter; $\mathrm{AC}-\mathrm{F} T=$ acre-feet, UG/L = micrograms per liter; T/DAY $=$ tons per day]

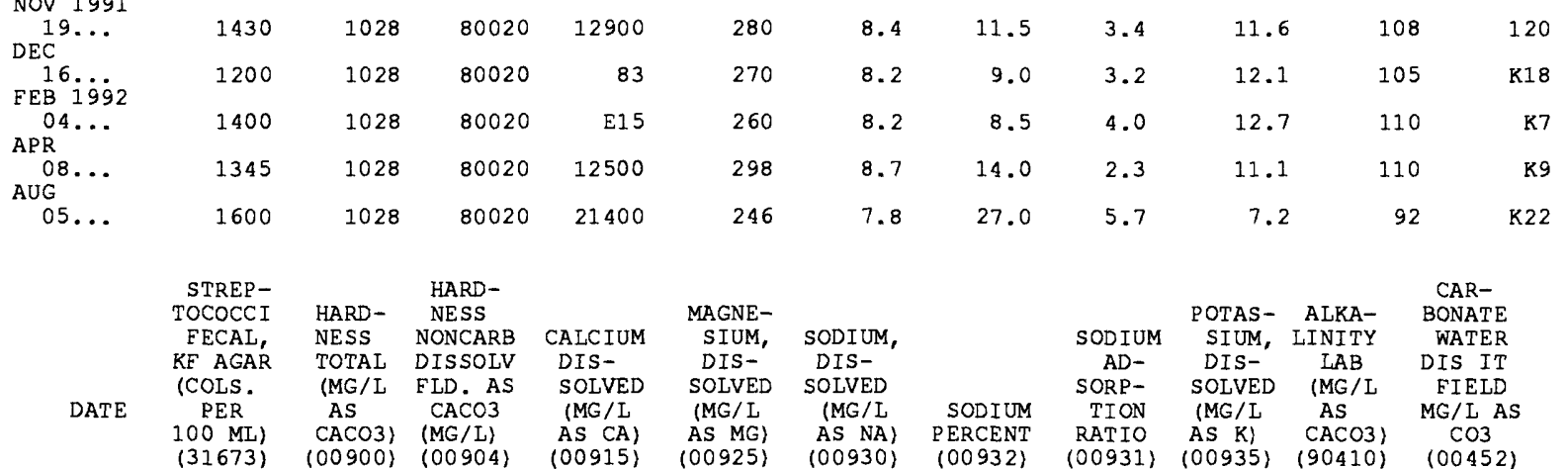

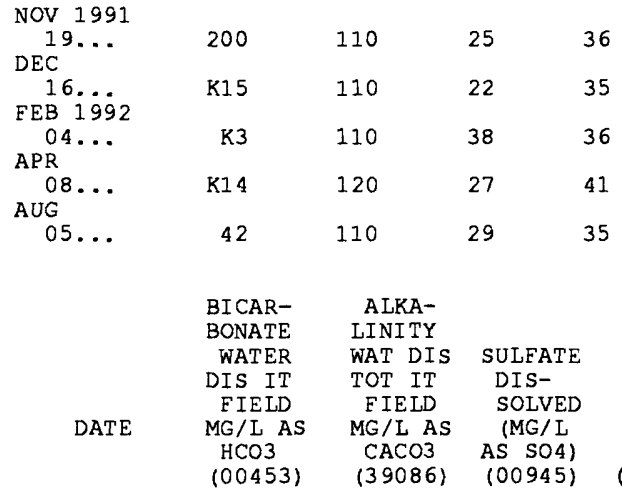

$\begin{array}{ccc}4.6 & 10 & 16 \\ 4.3 & 9.7 & 16 \\ 4.4 & 7.5 & 13 \\ 5.0 & 9.5 & 14 \\ 4.8 & 6.7 & 12\end{array}$

$\begin{array}{lllll}16 & 0.4 & 4.1 & 91 & 1 \\ 16 & .4 & 3.8 & 89 & 0 \\ 13 & .3 & 2.8 & 78 & 0 \\ 14 & .4 & 2.7 & 98 & 3 \\ 12 & .3 & 3.8 & 82 & 0\end{array}$

\begin{tabular}{|c|c|c|c|c|c|c|c|c|c|c|}
\hline $\begin{array}{c}\text { NOV } 1991 \\
19 . \ldots\end{array}$ & 100 & 84 & 29 & 12 & 0.10 & 0.80 & 143 & 148 & 4980 & 0.19 \\
\hline $\begin{array}{l}\text { DEC } \\
16 \\
\text { FEB }\end{array}$ & 101 & 82 & 31 & 13 & .20 & 1.2 & 170 & 149 & 38.1 & .23 \\
\hline $\begin{array}{l}04 \ldots \\
A P R\end{array}$ & 85 & 70 & 29 & 11 & .10 & 3.9 & 138 & 139 & -- & .19 \\
\hline$\underset{A U G}{08} \cdots$ & 111 & 96 & 34 & 14 & .20 & .20 & 170 & 165 & 5740 & .23 \\
\hline & 96 & 78 & 32 & 7.9 & .10 & 6.6 & 141 & 147 & 8150 & .19 \\
\hline
\end{tabular}


ARKANSAS RIVER BASIN

07193500 NEOSHO RIVER BELOW FORT GIBSON LAKE NEAR FORT GIBSON, OKLAHOMA--CONTINUED

WATER-QUALITY DATA, WATER YEAR OCTOBER 1991 TO SEPTEMBER 1992

\begin{tabular}{|c|c|c|c|c|c|c|c|c|c|c|}
\hline ATE & $\begin{array}{c}\text { NITRO- } \\
\text { GEN, } \\
\text { NITRATE } \\
\text { DIS- } \\
\text { SOLVED } \\
\text { (MG/I } \\
\text { AS N) } \\
(00618)\end{array}$ & $\begin{array}{c}\text { NITRO- } \\
\text { GEN, } \\
\text { NITRITE } \\
\text { TOTAL } \\
\text { (MG/L } \\
\text { AS N) } \\
(00615)\end{array}$ & $\begin{array}{c}\text { NITRO- } \\
\text { GEN, } \\
\text { NITRITE } \\
\text { DIS- } \\
\text { SOLVED } \\
\text { (MG/I } \\
\text { AS N) } \\
(00613)\end{array}$ & $\begin{array}{c}\text { NITRO- } \\
\text { GEN, } \\
\text { NO2+NO3 } \\
\text { TOTAL } \\
\text { (MG/I } \\
\text { AS N) } \\
(00630)\end{array}$ & $\begin{array}{c}\text { NITRO- } \\
\text { GEN, } \\
\text { NO2+NO3 } \\
\text { DIS- } \\
\text { SOLVED } \\
\text { (MG/L } \\
\text { AS N) } \\
(00631)\end{array}$ & $\begin{array}{l}\text { NITRO- } \\
\text { GEN, } \\
\text { AMMONIA } \\
\text { TOTAL } \\
\text { (MG/L } \\
\text { AS N) } \\
(00610)\end{array}$ & $\begin{array}{l}\text { NITRO- } \\
\text { GEN, } \\
\text { AMMONIA } \\
\text { DIS- } \\
\text { SOIVED } \\
\text { (MG/L } \\
\text { AS N) } \\
(00608)\end{array}$ & $\begin{array}{c}\text { NITRO- } \\
\text { GEN, } \\
\text { ORGANIC } \\
\text { TOTAL } \\
\text { (MG/L } \\
\text { AS N) } \\
(00605)\end{array}$ & $\begin{array}{l}\text { NITRO- } \\
\text { GEN, } \\
\text { TOTAL } \\
\text { (MG/L } \\
\text { AS N) } \\
(00600)\end{array}$ & $\begin{array}{c}\text { NITRO- } \\
\text { GEN, } \\
\text { NITRATE } \\
\text { TOTAL } \\
\text { (MG/L } \\
\text { AS N) } \\
(00620)\end{array}$ \\
\hline
\end{tabular}

\begin{tabular}{|c|c|c|c|c|c|c|c|c|c|c|}
\hline $\begin{array}{c}\text { NOV } 1991 \\
19 . . . \\
\text { DEC }\end{array}$ & -- & 0.010 & $<0.010$ & 0.150 & 0.150 & 0.020 & 0.020 & 0.38 & 0.55 & 0.140 \\
\hline FEB 16992 & 0.210 & .010 & .010 & .210 & .220 & .010 & $<.010$ & .39 & .61 & .200 \\
\hline $\begin{array}{l}04 \ldots \\
A R R\end{array}$ & .440 & .030 & .030 & -- & .470 & .010 & .020 & .39 & - & -- \\
\hline$\stackrel{08}{A U G}$ & -- & $<.010$ & $<.010$ & .073 & .079 & .040 & .030 & .46 & .57 & -- \\
\hline $05 \ldots$ & .540 & .030 & .020 & .590 & .560 & .050 & .030 & .25 & .89 & .560 \\
\hline DATE & $\begin{array}{c}\text { NITRO- } \\
\text { GEN, AM- } \\
\text { MONIA + } \\
\text { ORGANIC } \\
\text { TOTAL } \\
\text { (MG/L } \\
\text { AS N) } \\
(00625)\end{array}$ & $\begin{array}{c}\text { PHOS- } \\
\text { PHORUS } \\
\text { TOTAL } \\
\text { (MG/L } \\
\text { AS P) } \\
(00665)\end{array}$ & $\begin{array}{c}\text { PHOS- } \\
\text { PHORUS } \\
\text { DIS- } \\
\text { SOLVED } \\
\text { (MG/L } \\
\text { AS P) } \\
(00666)\end{array}$ & $\begin{array}{c}\text { PHOS- } \\
\text { PHORUS } \\
\text { ORTHO } \\
\text { TOTAL } \\
\text { (MG/L } \\
\text { AS P) } \\
(70507)\end{array}$ & $\begin{array}{l}\text { PHOS- } \\
\text { PHORUS } \\
\text { ORTHO, } \\
\text { DIS- } \\
\text { SOLVED } \\
\text { (MG/L } \\
\text { AS P) } \\
(00671)\end{array}$ & $\begin{array}{l}\text { ALUM- } \\
\text { INUM, } \\
\text { DIS- } \\
\text { SOLVED } \\
\text { (UG/L } \\
\text { AS AL) } \\
(01106)\end{array}$ & $\begin{array}{l}\text { BARIUM, } \\
\text { DIS- } \\
\text { SOLVED } \\
\text { (UG/L } \\
\text { AS BA) } \\
(01005)\end{array}$ & $\begin{array}{l}\text { COBALT, } \\
\text { DIS- } \\
\text { SOLVED } \\
\text { (UG/L } \\
\text { AS CO) } \\
(01035)\end{array}$ & $\begin{array}{l}\text { IRON, } \\
\text { DIS- } \\
\text { SOLVED } \\
\text { (UG/L } \\
\text { AS FE) } \\
(01046)\end{array}$ & $\begin{array}{c}\text { LITHIUM } \\
\text { DIS- } \\
\text { SOLVED } \\
\text { (UG/L } \\
\text { AS LI) } \\
(01130)\end{array}$ \\
\hline NOV 1991 & & & & & & & & & & \\
\hline${ }_{D E C}^{19} \cdots$ & 0.40 & 0.060 & 0.020 & -- & 0.020 & 20 & 51 & $<3$ & 9 & $<4$ \\
\hline FEB 16 9992 & .40 & .050 & $<.010$ & .020 & .020 & $<10$ & 47 & $<3$ & 6 & $<4$ \\
\hline$\stackrel{04 \ldots}{A P R}$ & .40 & .040 & .020 & .020 & .010 & 30 & 44 & $<3$ & 29 & $<4$ \\
\hline AUG & .50 & .030 & $<.010$ & .020 & $<.010$ & $<10$ & 46 & $<3$ & 6 & 5 \\
\hline $05 \ldots$ & .30 & .080 & .060 & .070 & .050 & 20 & 53 & $<3$ & 22 & $<4$ \\
\hline DATE & $\begin{array}{l}\text { MANGA- } \\
\text { NESE, } \\
\text { DIS- } \\
\text { SOLVED } \\
\text { (UG/L } \\
\text { AS MN) } \\
(01056)\end{array}$ & $\begin{array}{l}\text { MOLYB- } \\
\text { DENUM, } \\
\text { DIS- } \\
\text { SOLVED } \\
\text { (UG/L } \\
\text { AS MO) } \\
(01060)\end{array}$ & $\begin{array}{l}\text { NICKEL, } \\
\text { DIS- } \\
\text { SOLVED } \\
\text { (UG/L } \\
\text { AS NI) } \\
(01065)\end{array}$ & $\begin{array}{l}\text { SELE- } \\
\text { NIUM, } \\
\text { DIS- } \\
\text { SOLVED } \\
\text { (UG/L } \\
\text { AS SE) } \\
(01145)\end{array}$ & $\begin{array}{c}\text { SILVER, } \\
\text { DIS- } \\
\text { SOLVED } \\
\text { (UG/L } \\
\text { AS AG) } \\
(01075)\end{array}$ & $\begin{array}{c}\text { STRON- } \\
\text { TIUM, } \\
\text { DIS- } \\
\text { SOLVED } \\
\text { (UG/L } \\
\text { AS SR) } \\
(01080)\end{array}$ & $\begin{array}{l}\text { VANA- } \\
\text { DIUM, } \\
\text { DIS- } \\
\text { SOLVED } \\
\text { (UG/L } \\
\text { AS V) } \\
(01085)\end{array}$ & $\begin{array}{l}\text { SEDI- } \\
\text { MENT, } \\
\text { SUS- } \\
\text { PENDED } \\
(\mathrm{MG} / \mathrm{L}) \\
(80154)\end{array}$ & $\begin{array}{c}\text { SEDI- } \\
\text { MENT, } \\
\text { DIS- } \\
\text { CHARGE, } \\
\text { SUS- } \\
\text { PENDED } \\
\text { (T/DAY) } \\
(80155)\end{array}$ & $\begin{array}{c}\text { SED. } \\
\text { SUSP. } \\
\text { SIEVE } \\
\text { DIAM. } \\
\text { \% FINER } \\
\text { THAN } \\
.062 \mathrm{MM} \\
(70331)\end{array}$ \\
\hline NOV 1991 & & & & & & & & & & \\
\hline${ }_{\mathrm{DEC}}^{19} \ldots$ & 4 & $<10$ & $<1$ & $<1$ & $<1.0$ & 130 & $<6$ & 11 & 383 & 50 \\
\hline${ }_{\mathrm{FEB}}^{16} 19 \dot{9}_{2}$ & $<1$ & $<10$ & 1 & $<1$ & $<1.0$ & 120 & $<6$ & 16 & 3.6 & 56 \\
\hline $\begin{array}{l}04 \ldots \\
A P R\end{array}$ & 2 & $<10$ & $<1$ & $<1$ & $<1.0$ & 120 & $<6$ & 16 & -- & 67 \\
\hline AUG & $<1$ & $<10$ & 2 & $<1$ & $<1.0$ & 140 & $<6$ & 15 & 506 & 74 \\
\hline $05 \ldots$ & 7 & $<10$ & 3 & $<1$ & $<1.0$ & 160 & $<6$ & 40 & 2310 & 95 \\
\hline
\end{tabular}




\section{STREAMFLOW}

07194500 Arkansas River near Muskogee, Oklahoma

LOCATION.--Lat 3546'10", long 95'17'55", in NW1/4, sec.21, T.15 N., R.19 E., Muskogee County, at bridge on U.S. Highway 62, $1.7 \mathrm{mi}$ downstream from Neosho River, $3.5 \mathrm{mi}$ northeast of Muskogee.

DRAINAGE AREA.--96,674 $\mathrm{mi}^{2}$ of which $12,541 \mathrm{mi}^{2}$ probably is noncontributing.

REMARKS.--Gaging station discontinued Sept. 30, 1970, due to backwater conditions. Streamflow computed by combining flow at station 07165570 Arkansas River near Haskell, station 07176000 Verdigris River near Claremore, station 07178200 Bird Creek at State Highway 266 near Catoosa, station 07193000 Fort Gibson Lake Discharge near Fort Gibson, and estimating the flow for the ungaged intervening drainage area.

Monthly and yearly discharge

\begin{tabular}{lrr}
\hline Month & $\begin{array}{c}\text { Mean } \\
\left(\mathrm{ft}^{3} / \mathrm{s}\right)\end{array}$ & $\begin{array}{r}\text { Runoff in } \\
\text { acre-feet }\end{array}$ \\
\hline October & 3,896 & 239,600 \\
November & 9,504 & 565,500 \\
December & 22,825 & $1,403,000$ \\
January & 15,014 & 923,200 \\
February & 14,333 & 824,400 \\
March & 10,633 & 653,800 \\
April & 15,734 & 936,200 \\
May & 7,413 & 455,800 \\
June & 42,132 & $2,507,000$ \\
July & 46,443 & $2,856,000$ \\
August & 38,099 & $2,343,000$ \\
September & 16,886 & $1,005,000$ \\
& & $14,710,000$ \\
\hline Water Year 1992 & 20,267 & \\
\hline
\end{tabular}




\section{STREAMFLOW}

07195500 Illinois River near Watts, Oklahoma

LOCATION.--Lat $36^{\circ} 07^{\prime} 48^{\prime \prime}$, long 94 $34^{\prime} 19^{\prime \prime}$, in NW1/4NE1/4, sec.18, T.19 N., R.26 E., Adair County, near right bank on downstream side of bridge on U.S. Highway 59, 1.5 mi north of Watts, 4.5 mi downstream from Cincinnati Creek, and at mile 106.2.

DRAINAGE AREA.--635 mi².

AVERAGE DISCHARGE.--37 years, $605 \mathrm{ft}^{3} / \mathrm{s}$.

EXTREMES.--August 1955 to current year: Maximum discharge, $68,000 \mathrm{ft}^{3} / \mathrm{s} \mathrm{July} 25,1960$; minimum, 8.6 $\mathrm{ft}^{3} / \mathrm{s}$ Oct. 26, 1955, Sept. 19, Oct. 14, 1956.

REMARKS.--Records good. Since July 2, 1957, small diversion above station for municipal water supply for city of Siloam Springs, Arkansas. Satellite telemeter at station.

Monthly and yearly discharge

\begin{tabular}{lccccc}
\hline & $\begin{array}{c}\text { Total } \\
\left(\mathrm{ft}^{3} / \mathrm{s}\right)\end{array}$ & $\begin{array}{c}\text { Maximum } \\
\text { daily } \\
\left(\mathrm{ft}^{3} / \mathrm{s}\right)\end{array}$ & $\begin{array}{c}\text { Minimum } \\
\text { daily } \\
\left(\mathrm{ft}^{3} / \mathrm{s}\right)\end{array}$ & $\begin{array}{c}\text { Mean } \\
\left(\mathrm{ft}^{3} / \mathrm{s}\right)\end{array}$ & $\begin{array}{c}\text { Runoff in } \\
\text { acre-feet }\end{array}$ \\
\hline October & 13,944 & 2,910 & 103 & 450 & 27,660 \\
November & 44,526 & 4,890 & 333 & 1,484 & 88,320 \\
December & 48,036 & 7,030 & 629 & 1,550 & 95,280 \\
January & 17,048 & 917 & 362 & 550 & 33,810 \\
February & 16,190 & 1,440 & 297 & 558 & 32,110 \\
March & 10,018 & 420 & 262 & 323 & 19,870 \\
April & 11,674 & 1,260 & 208 & 389 & 23,160 \\
May & 14,361 & 1,230 & 185 & 463 & 28,490 \\
June & 36,040 & 7,780 & 349 & 1,201 & 71,490 \\
July & 15,980 & 2,520 & 205 & 515 & 31,700 \\
August & 20,203 & 3,170 & 201 & 652 & 40,070 \\
September & 8,339 & 730 & 159 & 278 & 16,540 \\
\hline Water Year 1992 & 256,359 & 7,780 & 103 & 700 & 508,500 \\
\hline
\end{tabular}




\section{ARKANSAS RIVER BASIN \\ 07195500 ILLINOIS RIVER NEAR WATTS, OKLAHOMA}

PERIOD OF RECORD.--October 1989 to current year.

REMARKS.--Samples were collected bi-monthly and specific conductance, $\mathrm{pH}$, water temperature, dissolved oxygen, and alkalinity were determined in the field.

\section{WATER-QUALITY DATA, WATER YEAR OCTOBER 1991 TO SEPTEMBER 1992}

[Five-digit numbers in parentheses are STORET parameter codes used for computer storage of data; $\mathrm{K}$, non-ideal count; US/CM = microsiemens per centimeter at 25 degrees Celsius; $\mathrm{NTU}=$ nephelometric turbidityunits; MG/L = milligrams per liter; MM = millimeters; UM$\mathrm{MF}=$ micrometer membrane filter; $\mathrm{AC}-\mathrm{ET}=$ acre-feet, UG/L = micrograms per liter; $\mathrm{T} / \mathrm{DAY}$ $=$ tons per day]

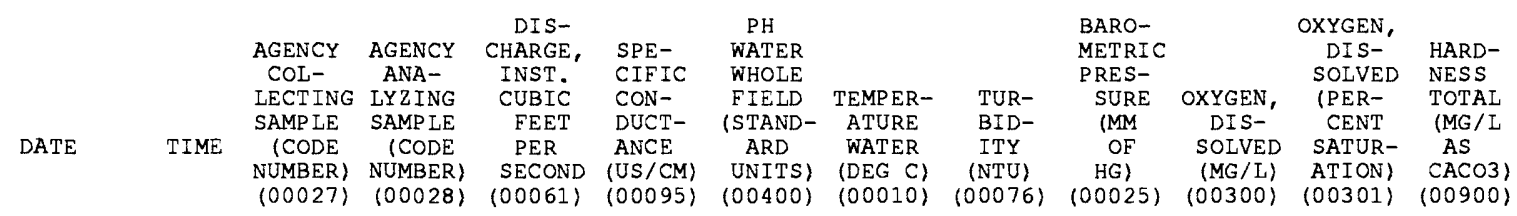

\begin{tabular}{|c|c|c|c|c|c|c|c|c|c|c|c|c|}
\hline $\begin{array}{l}\text { NOV } 1991 \\
19 \ddot{9} \\
\text { FEB } 1992\end{array}$ & 1015 & 1028 & 80020 & 2360 & 213 & 7.6 & 15.0 & 19 & 740 & 10.8 & 111 & 88 \\
\hline $\begin{array}{c}06 \ldots \\
\text { MAR }\end{array}$ & 1115 & 1028 & 80020 & 316 & 282 & 8.3 & 8.0 & -- & 740 & 12.3 & 107 & -- \\
\hline $\begin{array}{l}31 \\
\text { MAY }\end{array}$ & 1220 & 1028 & 80020 & 260 & 274 & 8.2 & 11.5 & 9.3 & 740 & 12.6 & 119 & 110 \\
\hline$\underset{A U G}{27} \cdots$ & 1040 & 1028 & 80020 & 393 & 257 & 8.3 & 17.0 & 8.0 & 740 & 8.8 & 94 & 110 \\
\hline $\operatorname{SEP}^{17} \cdots$ & 1519 & 1028 & 80020 & 355 & 301 & 8.0 & 22.5 & -- & 740 & 7.8 & 93 & -- \\
\hline $10 \ldots$ & 1145 & 1028 & 80020 & 299 & 307 & 7.9 & 22.0 & 23 & 740 & 8.0 & 94 & 120 \\
\hline
\end{tabular}

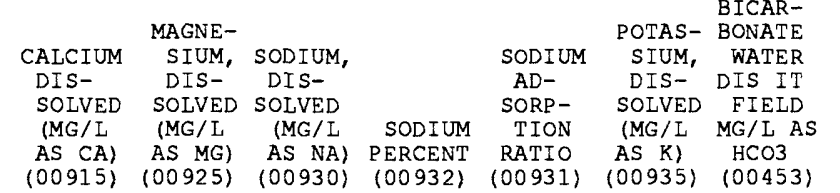

CAR-

BONATE CHLO- FLUOWATER DIS IT FIELD MG/L AS $\mathrm{CO} / \mathrm{L}$ AS
$\mathrm{CO}$

$\begin{array}{clc} & \text { CHLO- } & \text { FLUO- } \\ \text { SULFATE } & \text { RIDE, } & \text { RIDE, } \\ \text { DIS- } & \text { DIS- } & \text { DIS- } \\ \text { SOLVED } & \text { SOLVED } & \text { SOLVED } \\ \text { (MG/L } & \text { (MG/L } & \text { (MG/L } \\ \text { AS SO4) } & \text { AS CL) } & \text { AS F ) } \\ (00945) & (00940) & (00950)\end{array}$

\begin{tabular}{|c|c|c|c|c|c|c|c|c|}
\hline $\begin{array}{l}\text { NOV } 1991 \\
19.90 \\
\text { FEB } 1992\end{array}$ & 32 & 2.0 & 4.9 & 10 & 0.2 & 3.9 & 108 & 0 \\
\hline$\stackrel{06}{M A R}$ & -- & -- & -- & -- & -- & -- & 129 & 0 \\
\hline${ }_{\operatorname{MAY}}^{31} \ldots$ & 43 & 1.8 & 9.4 & 15 & .4 & 2.7 & 129 & 0 \\
\hline $\begin{array}{c}27 \\
\text { AUG }\end{array}$ & 42 & 2.0 & 7.5 & 12 & .3 & 3.0 & 133 & 0 \\
\hline $\operatorname{SEP}^{17} \ldots$ & -- & -- & $-\infty$ & -- & -- & -- & 140 & 0 \\
\hline $10 \ldots$ & 45 & 1.8 & 11 & 16 & .4 & 4.0 & 137 & 0 \\
\hline DATE & $\begin{array}{l}\text { SI LICA, } \\
\text { DIS- } \\
\text { SOLVED } \\
\text { (MG / L } \\
\text { AS } \\
\text { SIO2) } \\
(00955)\end{array}$ & $\begin{array}{l}\text { SOLIDS, } \\
\text { RESIDUE } \\
\text { AT } 180 \\
\text { DEG. C } \\
\text { DIS- } \\
\text { SOLVED } \\
(\mathrm{MG} / \mathrm{L}) \\
(70300)\end{array}$ & $\begin{array}{c}\text { SOLIDS, } \\
\text { SUM OF } \\
\text { CONSTI- } \\
\text { TUENTS, } \\
\text { DIS- } \\
\text { SOLVED } \\
(\mathrm{MG} / \mathrm{L}) \\
(70301)\end{array}$ & $\begin{array}{c}\text { SOLIDS, } \\
\text { DIS- } \\
\text { SOLVED } \\
\text { (TONS } \\
\text { PER } \\
\text { AC-FT) } \\
(70303 \text { ) }\end{array}$ & $\begin{array}{c}\text { SOLIDS, } \\
\text { DIS- } \\
\text { SOLVED } \\
\text { (TONS } \\
\text { PER } \\
\text { DAY) } \\
(70302)\end{array}$ & $\begin{array}{c}\text { NITRO- } \\
\text { GEN, } \\
\text { NO2+NO3 } \\
\text { DIS- } \\
\text { SOLVED } \\
\text { (MG/L } \\
\text { AS N) } \\
(00631)\end{array}$ & $\begin{array}{c}\text { NITRO- } \\
\text { GEN, } \\
\text { AMMONIA } \\
\text { DIS- } \\
\text { SOLVED } \\
\text { (MG/L } \\
\text { AS N) } \\
(00608)\end{array}$ & $\begin{array}{l}\text { NITRO- } \\
\text { GEN, } \\
\text { ORGANIC } \\
\text { TOTAL } \\
\text { (MG/L } \\
\text { AS N) } \\
(00605)\end{array}$ \\
\hline
\end{tabular}

$$
\begin{array}{ccc}
9.1 & 8.0 & 0.10 \\
-- & -- & -- \\
14 & 13 & <.10 \\
10 & 10 & .10 \\
-- & -- & -- \\
11 & 15 & <.10
\end{array}
$$

NOV 1991
$199 \ddot{9}$
FEB 1992
$\quad 06 \ldots$
MAR
$\quad 31 \ldots$
MAY
$\quad 27 \ldots$
AUG
$17 \ldots$
SEP
$\quad 10 \ldots$

$\begin{array}{rrrrrrrrrrr}9.1 & 163 & 142 & 0.22 & 1040 & 4.20 & 0.040 & 0.46 & 0.50 & 4.7 & 0.190 \\ -- & -- & -- & -- & -- & 2.70 & <.010 & .28 & .30 & 2.9 & .090 \\ 3.6 & 156 & 161 & .21 & 110 & 2.20 & .050 & .35 & .40 & 2.7 & .130 \\ 7.8 & 152 & 156 & .21 & 161 & 1.70 & .050 & .14 & .20 & 1.9 & .150 \\ -- & -- & -- & -- & -- & 2.00 & .040 & -- & <.20 & -- & .180 \\ 8.3 & 212 & 172 & .29 & 171 & 1.80 & .040 & .45 & .50 & 2.3 & .200\end{array}$


ARKANSAS RIVER BASIN

07195500 ILLINOIS RIVER NEAR WATTS, OKLAHOMA--CONTINUED

WATER-QUALITY DATA, WATER YEAR OCTOBER 1991 TO SEPTEMBER 1992

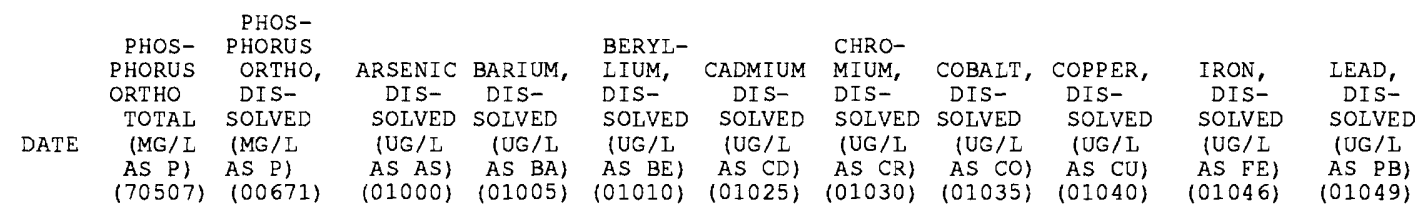

\begin{tabular}{|c|c|c|c|c|c|c|c|c|c|c|c|}
\hline $\begin{array}{l}\text { NOV } 1991 \\
19 . \ldots \\
\text { FEB } 1992\end{array}$ & -- & 0.130 & $<1$ & 47 & $<0.5$ & 6.0 & $<5$ & $<3$ & $<10$ & 380 & $<10$ \\
\hline $\begin{array}{l}06 \ldots \\
\text { MAR }\end{array}$ & 0.080 & .070 & -- & -- & -- & -- & -- & -- & -- & -- & -- \\
\hline $\begin{array}{c}31 \ldots \\
\text { MAY }\end{array}$ & .140 & .120 & $<1$ & 40 & $<.5$ & 1.0 & $<5$ & $<3$ & $<10$ & 13 & $<10$ \\
\hline${ }_{\text {AUG }}^{27} \cdots$ & .150 & .110 & 2 & 47 & $<.5$ & $<1.0$ & $<5$ & $<3$ & $<10$ & 7 & $<10$ \\
\hline $\operatorname{sep}^{17 \ldots}$ & .160 & .140 & -- & -- & -- & -- & -- & -- & -- & -- & -- \\
\hline $10 \ldots$ & .210 & .190 & 2 & 52 & $<.5$ & $<1.0$ & $<5$ & $<3$ & $<10$ & 13 & $<10$ \\
\hline
\end{tabular}

DATE

$\begin{array}{cc} & \\ & \text { MANGA- } \\ \text { LITHIUM } & \text { NESE, } \\ \text { DIS- } & \text { DIS- } \\ \text { SOLVED } & \text { SOLVED } \\ \text { (UG/L } & \text { (UG/L } \\ \text { AS LI) } & \text { AS MN) } \\ (01130) & (01056)\end{array}$

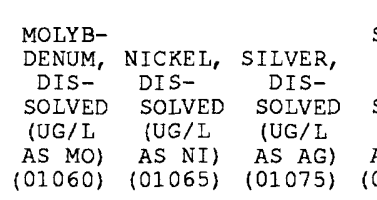

STRON- VANA-

TIUM, DIUM, ZTNC,

TIS- DIUM, ZINC,

$\begin{array}{lll}\text { DIS- } & \text { DIS- } & \text { DIS- } \\ \text { SOLVED } & \text { SOLVED } & \text { SOLVED }\end{array}$ (UG/L (UG/L (UG/I

AS SR) AS V) AS ZN)

MENT,

SUS-

PENDED

(MG/L)

(80154)

$\begin{array}{cc}\text { SEDI- } & \text { SED. } \\ \text { MENT, } & \text { SUSP. } \\ \text { DIS- } & \text { SIEVE } \\ \text { CHARGE, } & \text { DIAM. } \\ \text { SUS- } & \text { \% FINER } \\ \text { PENDED } & \text { THAN } \\ \text { (T/DAY) } & .062 \mathrm{MM} \\ (80155) & (70331)\end{array}$

\begin{tabular}{|c|c|c|c|c|c|c|c|c|c|c|c|}
\hline $\begin{array}{c}\text { NOV } 1991 \\
19.79 \\
\text { FEB } 1992\end{array}$ & $<4$ & 59 & $<10$ & $<10$ & $<1.0$ & 43 & $<6$ & 17 & 60 & 382 & 56 \\
\hline $\begin{array}{c}06 . . \\
\text { MAR }\end{array}$ & -- & -- & -- & -- & -- & -- & -- & -- & -- & -- & -- \\
\hline${ }_{M A Y}^{31} \ldots$ & $<4$ & 69 & $<10$ & $<10$ & $<1.0$ & 48 & $<6$ & 6 & -- & -- & -- \\
\hline AUG $27 \ldots$ & $<4$ & 60 & $<10$ & $<10$ & $<1.0$ & 49 & $<6$ & 5 & -- & -- & -- \\
\hline$\underset{S E P}{17 \ldots}$ & -- & -- & -- & -- & -- & -- & -- & -- & -- & -- & -- \\
\hline $10 \ldots$ & $<4$ & 59 & $<10$ & $<10$ & 2.0 & 51 & $<6$ & $<3$ & -- & -- & -- \\
\hline
\end{tabular}




\section{STREAMFLOW}

07195855 Flint Creek near West Siloam Springs, Oklahoma

LOCATION.--Lat $36^{\circ} 12^{\prime} 58^{\prime \prime}$, long 94³6' $15^{\prime \prime}$, in NE1/4NE1/4, sec.14, T.20 N., R.25 E., Delaware County, on left bank $180 \mathrm{ft}$ downstream from county bridge, $2.5 \mathrm{mi}$ from Arkansas-Oklahoma State line, northwest of Siloam Springs, Oklahoma.

DRAINAGE AREA. $--59.8 \mathrm{mi}^{2}$.

AVERAGE DISCHARGE.--13 years, $45.8 \mathrm{ft}^{3} / \mathrm{s}$.

EXTREMES.--June 1979 to current year: Maximum discharge, 5,590 $\mathrm{ft}^{3} / \mathrm{s} \mathrm{Dec.} \mathrm{21,} \mathrm{1984;} \mathrm{minimum} \mathrm{daily,}$ $0.40 \mathrm{ft}^{3} / \mathrm{s}$ Aug. 7, 1980.

REMARKS.--Records good.

Monthly and yearly discharge

\begin{tabular}{lccccc}
\hline Month & $\begin{array}{c}\text { Total } \\
\left(\mathrm{ft}^{3} / \mathrm{s}\right)\end{array}$ & $\begin{array}{c}\text { Maximum } \\
\text { daily } \\
\left(\mathrm{ft}^{3} / \mathrm{s}\right)\end{array}$ & $\begin{array}{c}\text { Minimum } \\
\text { daily } \\
\left(\mathrm{ft}^{3} / \mathrm{s}\right)\end{array}$ & $\begin{array}{c}\text { Mean } \\
\left(\mathrm{ft}^{3} / \mathrm{s}\right)\end{array}$ & $\begin{array}{r}\text { Runoff in } \\
\text { acre-feet }\end{array}$ \\
\hline October & 484 & 54 & 7.0 & 15.6 & 960 \\
November & 3,164 & 517 & 14 & 105 & 6,280 \\
December & 3,807 & 503 & 44 & 123 & 7,550 \\
January & 1,502 & 82 & 34 & 48.5 & 2,980 \\
February & 1,628 & 118 & 29 & 56.1 & 3,230 \\
March & 865 & 41 & 18 & 27.9 & 1,720 \\
April & 1,084 & 117 & 17 & 36.1 & 2,150 \\
May & 799 & 36 & 21 & 25.8 & 1,580 \\
June & 2,455 & 402 & 30 & 81.8 & 4,870 \\
July & 793 & 49 & 15 & 25.6 & 1,570 \\
August & 723 & 53 & 16 & 23.3 & 1,430 \\
September & 532 & 29 & 12 & 17.7 & 1,060 \\
& & & 7.0 & 48.7 & 35,380 \\
\hline Water Year 1992 & 17,836 & 517 & & & \\
\hline
\end{tabular}




\section{ARKANSAS RIVER BASIN \\ 07196000 FLINT CREEK NEAR KANSAS, OKLAHOMA}

LOCATION.--Lat 36¹1'11", long 94²'24", in SW1/4NW1/4, sec. 25, T.20 N., R.24 E., Deleware County, Hydrologic Unit 11110103, at U.S. Highway 412 bridge, $6.0 \mathrm{mi}$ southeast of Kansas, and at mi 2.2 .

DRAINAGE AREA--110 $\mathrm{mi}^{2}$.

PERIOD OF RECORD.--Water years 1955-61, 1963, 1975-80, July 1991 to current year.

REMARKS.--Samples were collected bi-monthly and specific conductance, $\mathrm{pH}$, water temperature, alkalinity, and dissolved oxygen were determined in the field.

WATER-QUALITY DATA, WATER YEAR OCTOBER 1991 TO SEPTEMBER 1992

[Five-digit numbers in parentheses are STORET parameter codes used for computer storage of data; $K$, non ideal count; US/CM = microsiemens per centimeter at 25 degrees Celsius; $\mathrm{NTU}=$ nephelometric turbidity units; MG/L = milligrams per liter; MM = millimeters; UM$\mathrm{MF}=$ micrometer membrane filter; $\mathrm{AC}-\mathrm{FT}=$ acre-feet, UG/L = micrograms per Iiter; $\mathrm{T} / \mathrm{DAY}$

= tons per day]

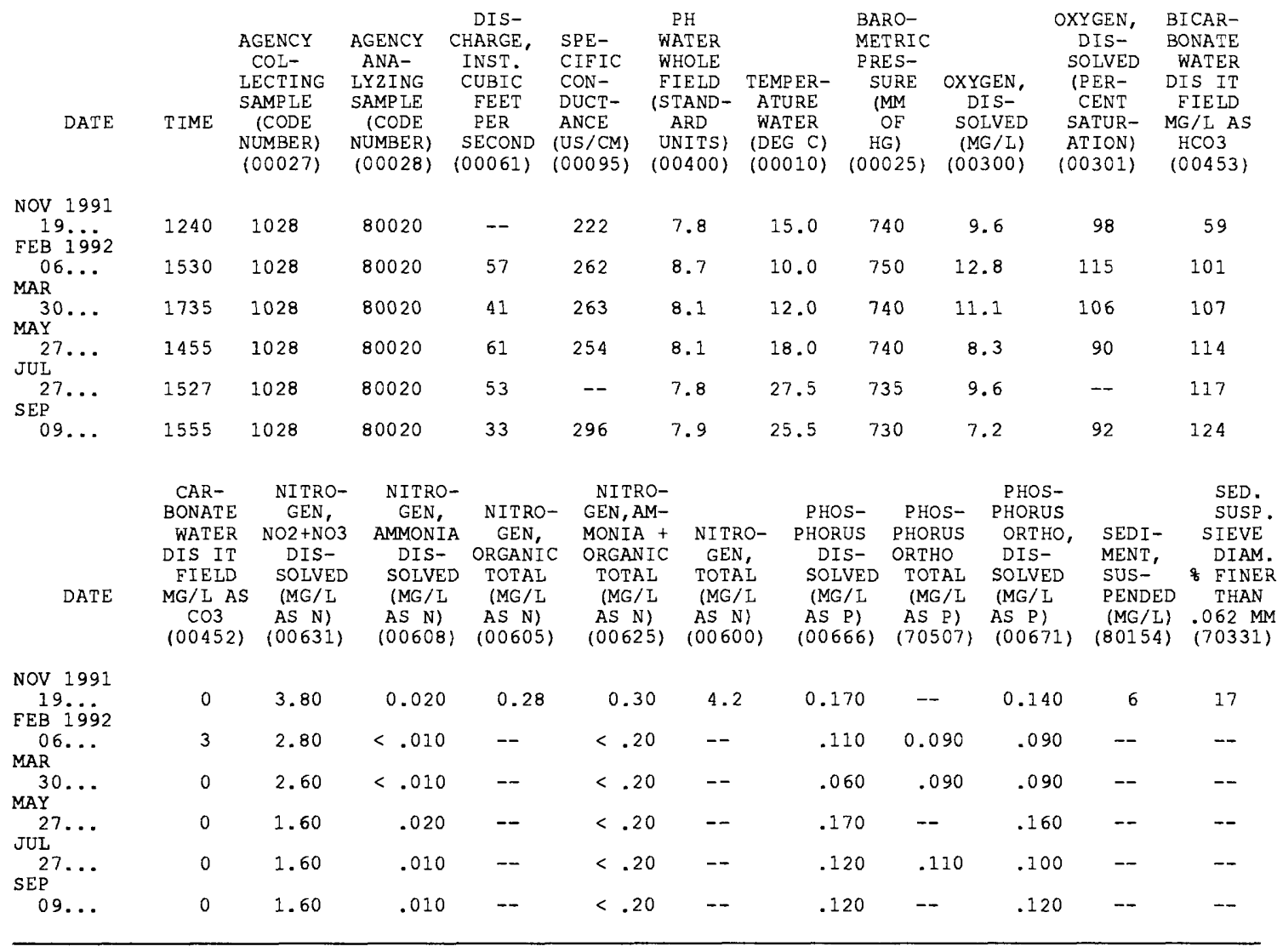




\section{ARKANSAS RIVER BASIN \\ 07196500 ILLINOIS RIVER NEAR TAHLEQUAH, OKLAHOMA}

LOCATION.--Lat 3555'22", long 94'55'24", in SE1/4NE1/4, sec.26, T.17 N., R.22 E., Cherokee County, Hydrologic Unit 11110103 , near center of channel on downstream side of pier of bridge, $0.2 \mathrm{mi}$ downstream from U.S. Highway $62,2.2 \mathrm{mi}$ northeast of Tahlequah, $6.5 \mathrm{mi}$ upstream from Baron Fork, and at mile 55.8.

DRATNAGE AREA.--959 $\mathrm{mi}^{2}$.

PERIOD OF RECORD.--Water years 1960-61, 1975-79, 1989 to current year.

REMARKS.--Samples were collected on a bimonthly schedule and specific conductance, $\mathrm{pH}$, water temperature, alkalinity, and dissolved oxygen were determined in the field.

WATER-QUALITY DATA, WATER YEAR OCTOBER 1991 TO SEPTEMBER 1992

[Five-digit numbers in parentheses are STORET parameter codes used for computer storage of data; $\mathrm{K}$, non-ideal count; US/CM = microsiemens per centimeter at 25 degrees Celsius; $\mathrm{NTU}=$ nephelometric turbidity units; MG/L = milligrams per liter; $M$ M = millimeters; UM$\mathrm{MF}=$ micrometer membrane filter; $\mathrm{AC}-\mathrm{FT}=$ acre-feet, $\mathrm{UG} / \mathrm{L}=$ micrograms per liter; $\mathrm{T} / \mathrm{DAY}$ $=$ tons per day]

\begin{tabular}{|c|c|c|c|c|c|c|c|c|c|c|c|}
\hline DATE & TIME & $\begin{array}{l}\text { AGENCY } \\
\text { COL- } \\
\text { LECTING } \\
\text { SAMPLE } \\
\text { (CODE } \\
\text { NUMBER) } \\
(00027)\end{array}$ & $\begin{array}{l}\text { AGENCY } \\
\text { ANA- } \\
\text { LYZING } \\
\text { SAMPLE } \\
\text { (CODE } \\
\text { NUMBER) } \\
(00028)\end{array}$ & $\begin{array}{c}\text { DIS- } \\
\text { CHARGE, } \\
\text { INST. } \\
\text { CUBIC } \\
\text { FEET } \\
\text { PER } \\
\text { SECOND } \\
(00061)\end{array}$ & $\begin{array}{l}\text { SPE- } \\
\text { CIFIC } \\
\text { CON- } \\
\text { DUCT- } \\
\text { ANCE } \\
(\text { US/CM) } \\
(00095)\end{array}$ & $\begin{array}{c}\text { PH } \\
\text { WATER } \\
\text { WHOLE } \\
\text { EIEID } \\
\text { (STAND- } \\
\text { ARD } \\
\text { UNITS) } \\
(00400)\end{array}$ & $\begin{array}{c}\text { TEMPER- } \\
\text { ATURE } \\
\text { WATER } \\
\text { (DEG C) } \\
(00010)\end{array}$ & $\begin{array}{c} \\
\text { TUR- } \\
\text { BID- } \\
\text { ITY } \\
(\text { NTU) } \\
(00076) \quad(0)\end{array}$ & $\begin{array}{l}\text { BARO- } \\
\text { METRIC } \\
\text { PRES- } \\
\text { SURE } \\
\text { (MM } \\
\text { OF } \\
\text { HG) } \\
(00025) \quad 10\end{array}$ & $\begin{array}{cc} & \text { OX } \\
& S \\
\text { OXYGEN, } & ( \\
\text { DIS- } & \\
\text { SOLVED } & S \\
(\text { MG } / L) & A \\
(00300) & 10\end{array}$ & $\begin{array}{l}\text { OXYGEN, } \\
\text { DIS- } \\
\text { SOLVED } \\
\text { (PER- } \\
\text { CENT } \\
\text { SATUR- } \\
\text { ATION) } \\
\text { (O0301) }\end{array}$ \\
\hline $\begin{array}{c}\text { OV } 1991 \\
20 \ldots\end{array}$ & 1300 & 1028 & 80020 & 3850 & 198 & 7.8 & 13.5 & 21 & 750 & 9.8 & 96 \\
\hline${ }_{E B}^{E C} 10 . .992$ & 1600 & 1028 & 80020 & 1380 & 225 & 7.9 & 12.0 & -- & 750 & 11.1 & 105 \\
\hline $\begin{array}{l}05 \ldots \\
P R\end{array}$ & 1600 & 1028 & 80020 & 482 & 246 & 8.6 & 9.0 & $\ldots$ & 750 & 13.0 & 114 \\
\hline $\begin{array}{l}09 . . \\
\text { JUN }\end{array}$ & 1900 & 1028 & 80020 & 354 & 250 & 8.7 & 20.0 & 2.5 & 745 & 12.8 & 144 \\
\hline $08 .$. & 1630 & 1028 & 80020 & 7200 & 180 & 7.7 & 20.0 & 45 & 745 & 8.0 & 90 \\
\hline $29 \ldots$ & 1330 & 1028 & 80020 & 1290 & 208 & 7.7 & 26.5 & 27 & 745 & 7.0 & 89 \\
\hline DATE & $\begin{array}{l}\text { HARD- } \\
\text { NESS } \\
\text { TOTAL } \\
\text { (MG/L } \\
\text { AS } \\
\text { CACO3) } \\
(00900)\end{array}$ & $\begin{array}{c}\text { CALCIUM } \\
\text { DIS- } \\
\text { SOLVED } \\
\text { (MG/L } \\
\text { AS CA) } \\
(00915)\end{array}$ & $\begin{array}{c}\text { MAGNE- } \\
\text { SIUM, } \\
\text { DIS- } \\
\text { SOLVED } \\
\text { (MG/L } \\
\text { AS MG) } \\
(00925)\end{array}$ & $\begin{array}{l}\text { SODIUM, } \\
\text { DIS- } \\
\text { SOLVED } \\
\text { (MG /L } \\
\text { AS NA) } \\
(00930)\end{array}$ & $\begin{array}{l}\text { SODIUM } \\
\text { PERCENT } \\
(00932)\end{array}$ & $\begin{array}{c}\text { SODIUM } \\
\text { AD- } \\
\text { SORP- } \\
\text { TION } \\
\text { RATIO } \\
(00931)\end{array}$ & $\begin{array}{c}\text { POTAS- } \\
\text { SIUM, } \\
\text { DIS- } \\
\text { SOLVED } \\
\text { (MG/L } \\
\text { AS K) } \\
(00935)\end{array}$ & $\begin{array}{l}\text { BICAR- } \\
\text { BONATE } \\
\text { WATER } \\
\text { DIS IT } \\
\text { FIELD } \\
\text { MG/L AS } \\
\text { HCO3 } \\
(00453)\end{array}$ & $\begin{array}{c}\text { CAR- } \\
\text { BONATE } \\
\text { WATER } \\
\text { DIS IT } \\
\text { FIELD } \\
\text { MG/L AS } \\
\text { CO3 } \\
(00452)\end{array}$ & $\begin{array}{l}\text { SULFATE } \\
\text { DIS- } \\
\text { SOLVED } \\
\text { (MG/L } \\
\text { AS SO4) } \\
(00945)\end{array}$ & $\begin{array}{l}\text { CHLO- } \\
\text { RIDE, } \\
\text { DIS- } \\
\text { SOLVED } \\
\text { (MG/L } \\
\text { AS CL) } \\
(00940)\end{array}$ \\
\hline
\end{tabular}

\begin{tabular}{|c|c|c|c|c|c|c|c|c|c|c|c|}
\hline $\begin{array}{c}\text { Nov } 1991 \\
20 \ldots\end{array}$ & 82 & 30 & 1.8 & 4.6 & 10 & 0.2 & 3.2 & 77 & 0 & 9.6 & 7.4 \\
\hline $\begin{array}{l}\text { DEC } \\
10\end{array}$ & -- & -- & -- & -- & - & -- & -- & 95 & 0 & -- & -- \\
\hline $\begin{array}{l}\text { FEB } 1992 \\
05 \cdots \\
\text { APR }\end{array}$ & -- & -- & -- & $\ldots$ & -- & -- & -- & 106 & 2 & -- & -- \\
\hline $09 .$. & 110 & 40 & 1.9 & 8.2 & 14 & .3 & 2.6 & 108 & 2 & 14 & 13 \\
\hline $\begin{array}{l}08 . \\
\text { JUL }\end{array}$ & 80 & 29 & 1.8 & 4.0 & 9 & .2 & 3.2 & 73 & 0 & 7.7 & 2.8 \\
\hline $29 \ldots$ & 89 & 33 & 1.6 & 5.8 & 12 & .3 & 3.8 & 99 & 0 & 8.1 & 8.8 \\
\hline
\end{tabular}

\begin{tabular}{ccccccc}
\multicolumn{1}{c}{ SOLIDS, } & SOLIDS, & & NITRO- \\
FLUO- & SILICA, & RESIDUE & SUM OF & SOLIDS, SOLIDS, & GEN, \\
RIDE, & DIS- & AT 180 & CONSTI- & DIS- & DIS- & NO2+NO3 \\
DIS- & SOLVED & DEG. C TUENTS, & SOLVED & SOLVED & DIS- \\
SOLVED & (MG/L & DIS- & DIS- & (TONS & (TONS & SOLVED \\
$(M G / L$ & AS & SOLVED & SOLVED & PER & PER & (MG/L \\
AS F) & SIO2) & $($ MG/L) & $($ MG/L) & AC-ET) & DAY) & AS N) \\
$(00950)$ & $(00955)$ & $(70300)$ & $(70301)$ & $(70303)$ & $(70302)$ & $(00631)$
\end{tabular}

$\begin{array}{cc}\text { NITRO- } & \\ \text { GEN, } & \text { NITRO- } \\ \text { AMMONIA } & \text { GEN, } \\ \text { DIS- } & \text { ORGANIC } \\ \text { SOLVED } & \text { TOTAL } \\ \text { (MG/L } & \text { (MG/L } \\ \text { AS N) } & \text { AS N) } \\ (00608) & (00605)\end{array}$

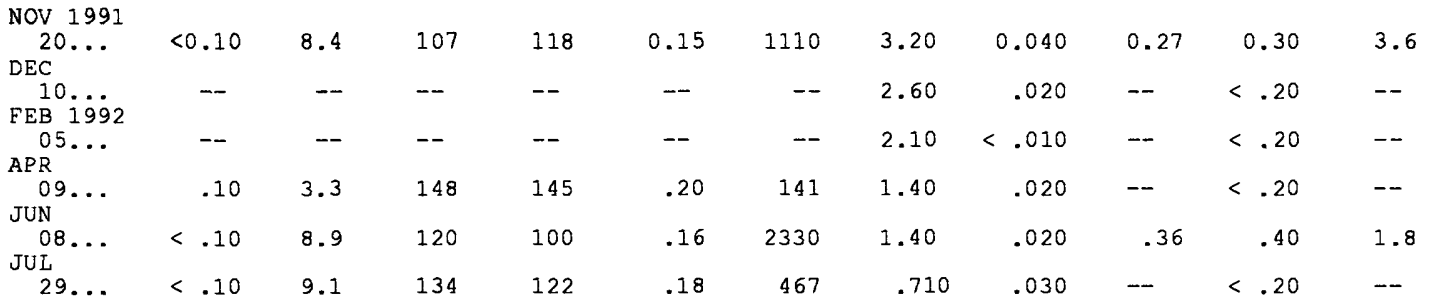


ARKANSAS RIVER BASIN

07196500 ILLINOIS RIVER NEAR TAHLEQUAH, OKLAHOMA--CONTINUED

WATER-QUALITY DATA, WATER YEAR OCTOBER 1991 TO SEPTEMBER 1992

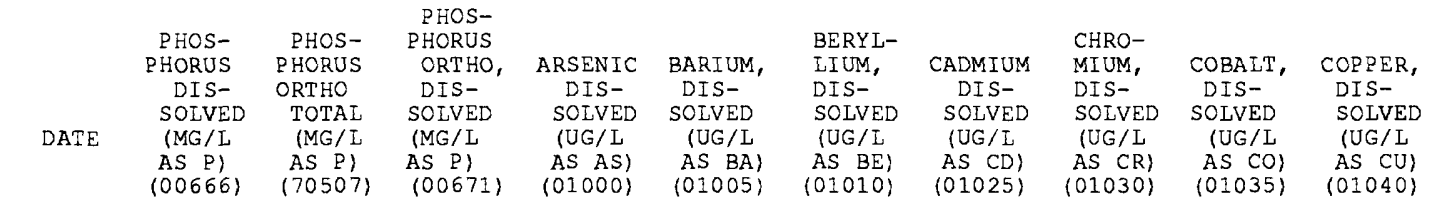

\begin{tabular}{|c|c|c|c|c|c|c|c|c|c|c|}
\hline $\begin{array}{c}\text { NOV } 1991 \\
20 \ldots \\
\text { DEC }\end{array}$ & 0.100 & 0.090 & 0.090 & $<1$ & 37 & $<0.5$ & 1.0 & $<5$ & $<3$ & $<10$ \\
\hline FEB $10 . \ddot{992}$ & .030 & .030 & .020 & -- & -- & -- & -- & -- & -- & -- \\
\hline $\begin{array}{c}05 \cdots \\
A P R\end{array}$ & $<.010$ & .040 & .030 & -- & -- & -- & -- & -- & -- & -- \\
\hline $\begin{array}{l}09 . . \\
\text { JUN }\end{array}$ & .060 & .050 & .050 & 1 & 40 & $<.5$ & $<1.0$ & $<5$ & $<3$ & $<10$ \\
\hline $\begin{array}{l}08 \ldots \\
\text { JUL }\end{array}$ & .120 & .160 & .120 & $<1$ & 52 & $<.5$ & $<1.0$ & $<5$ & $<3$ & $<10$ \\
\hline $29 \ldots$ & .010 & .040 & .020 & $<1$ & 42 & $<.5$ & $<1.0$ & $<5$ & $<3$ & $<10$ \\
\hline DATE & $\begin{array}{l}\text { IRON, } \\
\text { DIS- } \\
\text { SOLVED } \\
\text { (UG/L } \\
\text { AS FE) } \\
(01046)\end{array}$ & $\begin{array}{l}\text { LEAD, } \\
\text { DIS- } \\
\text { SOLVED } \\
\text { (UG/L } \\
\text { AS PB) } \\
(01049)\end{array}$ & $\begin{array}{l}\text { LITHIUM } \\
\text { DIS- } \\
\text { SOLVED } \\
\text { (UG/L } \\
\text { AS LI) } \\
(01130)\end{array}$ & $\begin{array}{l}\text { MANGA- } \\
\text { NESE, } \\
\text { DIS- } \\
\text { SOLVED } \\
\text { (UG / } \\
\text { AS MN) } \\
(01056 \text { ) }\end{array}$ & $\begin{array}{l}\text { MOLYB- } \\
\text { DENUM, } \\
\text { DIS- } \\
\text { SOLVED } \\
\text { (UG/L } \\
\text { AS MO) } \\
(01060)\end{array}$ & $\begin{array}{l}\text { NICKEL, } \\
\text { DIS- } \\
\text { SOLVED } \\
\text { (UG/I } \\
\text { AS NI) } \\
(01065)\end{array}$ & $\begin{array}{c}\text { SILVER, } \\
\text { DIS- } \\
\text { SOLVED } \\
\text { (UG/L } \\
\text { AS AG) } \\
(01075)\end{array}$ & $\begin{array}{c}\text { STRON- } \\
\text { TIUM, } \\
\text { DIS- } \\
\text { SOLVED } \\
\text { (UG/L } \\
\text { AS SR) } \\
(01080)\end{array}$ & $\begin{array}{l}\text { VANA- } \\
\text { DIUM, } \\
\text { DIS- } \\
\text { SOLVED } \\
\text { (UG/L } \\
\text { AS V) } \\
(01085)\end{array}$ & $\begin{array}{c}\text { ZINC, } \\
\text { DIS- } \\
\text { SOLVED } \\
\text { (UG/L } \\
\text { AS ZN) } \\
\{01090\}\end{array}$ \\
\hline
\end{tabular}

\begin{tabular}{|c|c|c|c|c|c|c|c|c|c|c|}
\hline $\begin{array}{c}\text { NOV } 1991 \\
20 \ldots \\
\text { DEC }\end{array}$ & 31 & $<10$ & $<4$ & 5 & $<10$ & $<10$ & 1.0 & 38 & $<6$ & 14 \\
\hline${ }_{\mathrm{FEB}}^{10} \ddot{1} \ddot{9} 2$ & -- & -- & -- & -- & -- & -- & -- & - & -- & -- \\
\hline $\begin{array}{l}05 \ldots \\
A P R\end{array}$ & -- & - & -- & -- & -- & -- & -- & - & - & -- \\
\hline${ }_{\text {JUN }}^{09} \ldots$ & 4 & $<10$ & $<4$ & 7 & $<10$ & $<10$ & $<1.0$ & 48 & $<6$ & 4 \\
\hline JUL & 86 & $<10$ & $<4$ & 6 & $<10$ & $<10$ & $<1.0$ & 39 & $<6$ & 9 \\
\hline $29 \ldots$ & 11 & $<10$ & $<4$ & 4 & $<10$ & $<10$ & $<1.0$ & 42 & $<6$ & 5 \\
\hline
\end{tabular}




\section{STREAMFLOW}

07196900 Baron Fork at Dutch Mills, Arkansas

LOCATION.--Lat $35^{\circ} 52^{\prime} 48^{\prime \prime}$, long $94^{\circ} 29^{\prime} 11^{\prime \prime}$, on line between secs.21 and 22, T.14 N., R.33 W., Washington County, near right bank on downstream side of bridge on State Highway 59 at Dutch Mills, $2.2 \mathrm{mi}$ downstream from Fly Creek, and $2.9 \mathrm{mi}$ upstream from Arkansas-Oklahoma State line.

DRAINAGE AREA.- $-40.6 \mathrm{mi}^{2}$.

AVERAGE DISCHARGE.--34 years, $42.6 \mathrm{ft}^{3} / \mathrm{s}$.

EXTREMES.--April 1958 to current year: Maximum discharge, 20,900 $\mathrm{ft}^{3} / \mathrm{s}$, Nov. 18, 1985; no flow at times.

REMARKS.--Records good.

Monthly and yearly discharge

\begin{tabular}{lccccr}
\hline Month & $\begin{array}{c}\text { Total } \\
\left(\mathrm{ft}^{3} / \mathrm{s}\right)\end{array}$ & $\begin{array}{c}\text { Maximum } \\
\text { daily } \\
\left(\mathrm{ft}^{3} / \mathrm{s}\right)\end{array}$ & $\begin{array}{c}\text { Minimum } \\
\text { daily } \\
\left(\mathrm{ft}^{3} / \mathrm{s}\right)\end{array}$ & $\begin{array}{c}\text { Mean } \\
\left(\mathrm{ft}^{3} / \mathrm{s}\right)\end{array}$ & $\begin{array}{c}\text { Runoff in } \\
\text { acre-feet }\end{array}$ \\
\hline October & 1,300 & 372 & 1.1 & 41.9 & 2,580 \\
November & 1,439 & 286 & 17 & 48.0 & 2,850 \\
December & 2,912 & 424 & 33 & 93.9 & 5,780 \\
January & 907 & 57 & 17 & 29.3 & 1,800 \\
February & 592 & 47 & 12 & 20.4 & 1,170 \\
March & 521 & 32 & 12 & 16.8 & 1,030 \\
April & 537 & 108 & 8.2 & 17.9 & 1,070 \\
May & 1,343 & 231 & 4.9 & 43.3 & 2,660 \\
June & 2,123 & 486 & 13 & 70.8 & 4,210 \\
July & 479 & 89 & 5.1 & 15.5 & 951 \\
August & 1,922 & 1,170 & 4.8 & 62 & 3,810 \\
September & 532 & 139 & 4.2 & 17.7 & 1,060 \\
& & & & & 28,970 \\
\hline Water Year 1992 & 14,607 & 1,170 & 1.1 & 39.9 & \\
\hline
\end{tabular}




\section{ARKANSAS RIVER BASIN \\ 07197000 BARON FORK AT ELDON, OKLAHOMA}

LOCATION.--Lat 35 $55^{\prime} 16^{\prime \prime}$, long 94 50'18", in NE1/4SE1/4, sec.27, T.17 N., R.23 E., Cherokee County, Hydrologic Unit 11110103, on downstream left abutment of bridge on State Highway $51,0.4 \mathrm{mi}$ southeast of Eldon, $6.0 \mathrm{mi}$ downstream from Tyner Creek, and at mile 8.8 .

DRAINAGE AREA --307 $\mathrm{mi}^{2}$.

PERIOD OF RECORD.--1948, 1958-60, 1991 to current year.

REMARKS.--Samples were collected bimonthly and specific conductance, $\mathrm{pH}$, water temperature, and dissolved oxygen were determined in the field.

WATER-QUALITY DATA, WATER YEAR OCTOBER 1991 TO SEPTEMBER 1992

[Five-digit numbers in parentheses are STORET parameter codes used for computer storage of data;K, non-ideal count; US/CM = microsiemens per centimeter at 25 degrees Celsius: $\mathrm{NTU}=$ nephelometric turbidity units; MG/L = milligrams per liter; MM = millimeters; UM$\mathrm{MF}=$ micrometer membrane filter; $\mathrm{AC}-\mathrm{FT}=$ acre-feet, UG/L = micrograms per liter; T/DAY = tons per day]

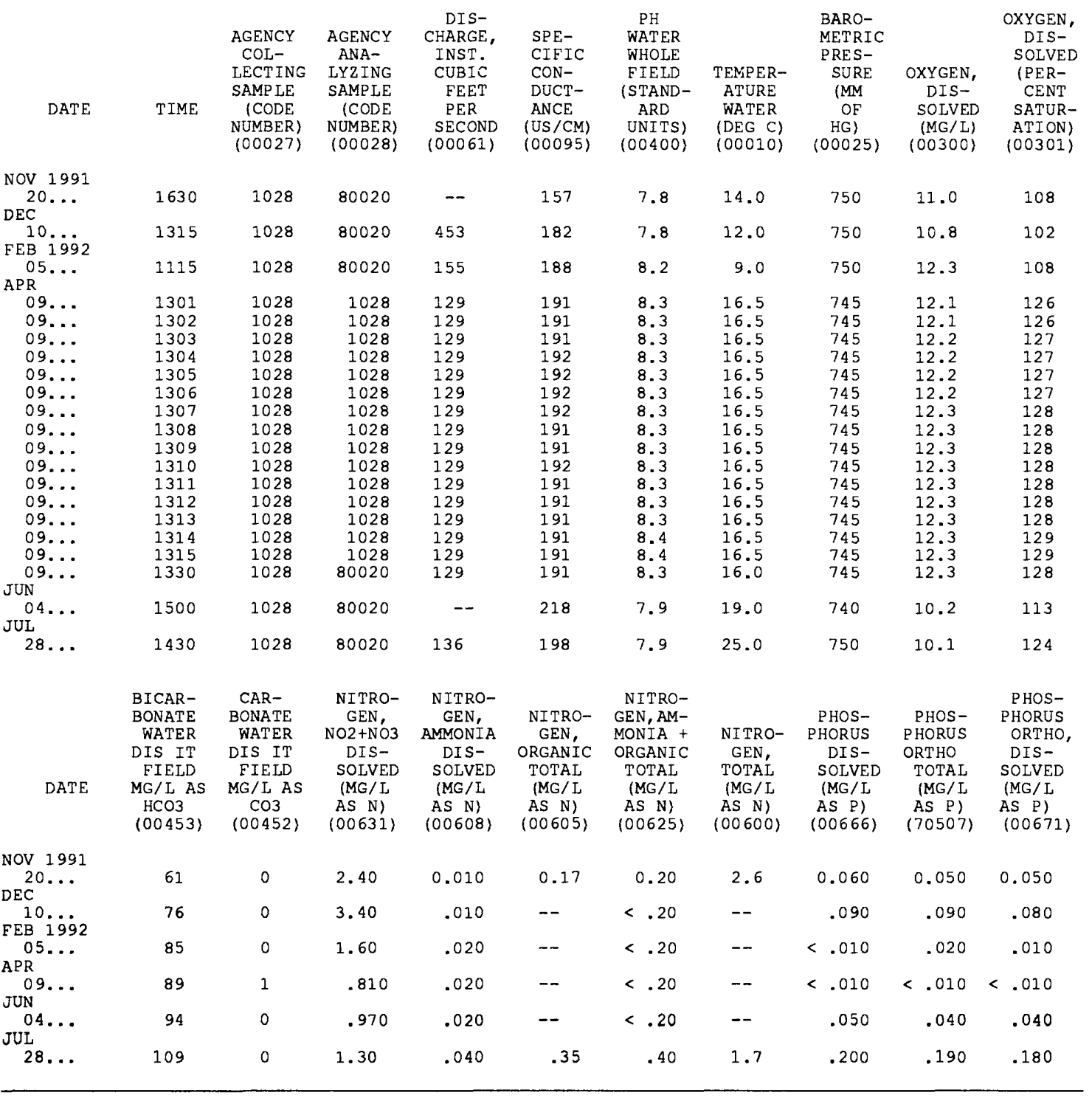




\section{ARKANSAS RIVER BASIN \\ 07198000 ILLINOIS RIVER NEAR GORE, OKLAHOMA}

LOCATION.--Lat 35³4'23", long 9504'07", in NE1/4SW1/4, sec.27, T.13 N., R.21 E., Sequoyah County, Hydrologic Unit 11110104, on right bank 4.2 mi downstream from Tenkiller Ferry Dam, 4.5 mi northeast of Gore, and at mile 8.5.

DRAINAGE AREA- $-1,626 \mathrm{mi}^{2}$.

PERIOD OF RECORD.--Water years 1948, 1952, 1954 to current year.

PERIOD OF DAILY RECORD.--

SPECIFIC CONDUCTANCE: October 1947 to September 1948, October 1953 to September 1963.

WATER TEMPERATURE: October 1947 to September 1948, October 1953 to September 1963.

REMARKS.--Samples were collected bimonthly and specific conductance, $\mathrm{pH}$, water temperature, alkalinity, and dissolved oxygen were determined in the field.

WATER-QUALITY DATA, WATER YEAR OCTOBER 1991 TO SEPTEMRER 1992

[Five-digit numbers in parentheses are STORET parameter codes used for computer storage of data; K, non-ideal count; US/CM = microsiemens per centimeter at 25 degrees Celsius; $\mathrm{NTU}=$ nephelometric turbidity units; $\mathrm{MG} / \mathrm{L}=\mathrm{milligrams}$ per liter; $\mathrm{MM}=\mathrm{millimeters;}$ UM$\mathrm{MF}=$ micrometer membrane filter; $\mathrm{AC}-\mathrm{FT}=$ acre-feet, $\mathrm{UG} / \mathrm{L}=$ micrograms per $1 \mathrm{iter} ; \mathrm{T} / \mathrm{DAY}$ $=$ tons per day]

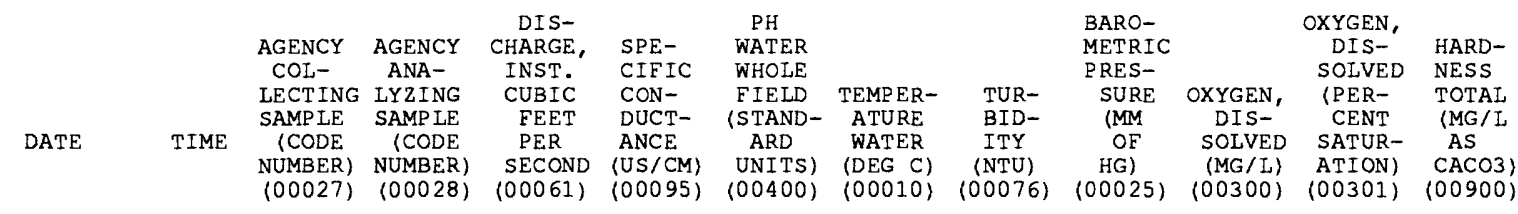

\begin{tabular}{|c|c|c|c|c|c|c|c|c|c|c|c|c|}
\hline $\begin{array}{c}\text { OCT } 1991 \\
09 . \ldots \\
\text { DEC }\end{array}$ & 0815 & 1028 & 80020 & 12 & 350 & 7.8 & 15.5 & 1.2 & 757 & 5.4 & 54 & -- \\
\hline MAR 17992 & 0800 & 1028 & 80020 & 3930 & 189 & 7.8 & 10.5 & -- & 759 & 9.1 & 82 & -- \\
\hline$\underset{A P R}{04 \ldots}$ & 0815 & 1028 & 80020 & 1670 & 205 & 8.0 & 9.5 & 1.5 & 747 & 10.2 & 91 & 83 \\
\hline$\underset{\text { JUN }}{08 \ldots}$ & 1445 & 1028 & 80020 & 1910 & 191 & 8.3 & 11.5 & -- & 750 & 9.9 & 92 & -- \\
\hline${ }_{\text {JUL }}^{03 \ldots}$ & 1145 & 1028 & 80020 & 4080 & 195 & 7.5 & 13.0 & .70 & 744 & 6.6 & 64 & 89 \\
\hline $\begin{array}{l}16 \ldots \\
16 \ldots \\
16 \ldots \\
16 \ldots \\
16 \ldots \\
16 \ldots \\
16 \ldots \\
16 \ldots \\
16 \ldots \\
16 \ldots\end{array}$ & $\begin{array}{l}0652 \\
0655 \\
0657 \\
0700 \\
0702 \\
0705 \\
0707 \\
0710 \\
0712 \\
0714\end{array}$ & $\begin{array}{l}1028 \\
1028 \\
1028 \\
1028 \\
1028 \\
1028 \\
1028 \\
1028 \\
1028 \\
1028\end{array}$ & $\begin{array}{l}1028 \\
1028 \\
1028 \\
1028 \\
1028 \\
1028 \\
1028 \\
1028 \\
1028 \\
1028\end{array}$ & $\begin{array}{l}77 \\
77 \\
77 \\
77 \\
77 \\
77 \\
77 \\
77 \\
77 \\
77\end{array}$ & $\begin{array}{l}259 \\
258 \\
258 \\
259 \\
260 \\
260 \\
260 \\
260 \\
260 \\
256\end{array}$ & $\begin{array}{l}6.9 \\
7.1 \\
7.2 \\
7.2 \\
7.2 \\
7.2 \\
7.2 \\
7.2 \\
7.3 \\
7.3\end{array}$ & $\begin{array}{l}17.0 \\
17.0 \\
17.0 \\
17.0 \\
17.0 \\
17.0 \\
17.0 \\
17.0 \\
17.0 \\
17.5\end{array}$ & $\begin{array}{l}-- \\
-- \\
-- \\
-- \\
-- \\
-- \\
-- \\
--\end{array}$ & $\begin{array}{l}745 \\
745 \\
745 \\
745 \\
745 \\
745 \\
745 \\
745 \\
745 \\
745\end{array}$ & $\begin{array}{l}3.4 \\
3.8 \\
3.9 \\
4.0 \\
4.0 \\
4.0 \\
4.0 \\
3.8 \\
3.8 \\
3.8\end{array}$ & $\begin{array}{l}36 \\
40 \\
41 \\
42 \\
42 \\
42 \\
42 \\
40 \\
40 \\
41\end{array}$ & $\begin{array}{l}-- \\
-- \\
-- \\
-- \\
-- \\
-- \\
-- \\
--\end{array}$ \\
\hline $\begin{array}{l}\text { AUG } \\
19 . .\end{array}$ & 1330 & 1028 & 80020 & 151 & 242 & 7.8 & 20.0 & 2.0 & 755 & 9.9 & 110 & 100 \\
\hline DATE & $\begin{array}{l}\text { CALCIUM } \\
\text { DIS- } \\
\text { SOLVED } \\
\text { (MG/L } \\
\text { AS CA) } \\
(00915)\end{array}$ & $\begin{array}{c}\text { MAGNE- } \\
\text { SIUM, } \\
\text { DIS- } \\
\text { SOLVED } \\
\text { (MG/I } \\
\text { AS MG) } \\
(00925)\end{array}$ & $\begin{array}{c}\text { SODIUM, } \\
\text { DIS- } \\
\text { SOLVED } \\
\text { (MG/L } \\
\text { AS NA) } \\
(00930)\end{array}$ & $\begin{array}{l}\text { SODIUM } \\
\text { PERCENT } \\
(00932)\end{array}$ & $\begin{array}{c}\text { SODIUM } \\
\text { AD- } \\
\text { SORP- } \\
\text { TION } \\
\text { RATIO } \\
(00931)\end{array}$ & $\begin{array}{c}\text { POTAS- } \\
\text { SIUM, } \\
\text { DIS- } \\
\text { SOLVED } \\
\text { (MG/L } \\
\text { AS K) } \\
(00935)\end{array}$ & $\begin{array}{l}\text { BICAR- } \\
\text { BONATE } \\
\text { WATER } \\
\text { DIS IT } \\
\text { FIELD } \\
\text { MG/L AS } \\
\text { HCO3 } \\
(00453)\end{array}$ & $\begin{array}{c}\text { CAR- } \\
\text { BONATE } \\
\text { WATER } \\
\text { DIS IT } \\
\text { FIELD } \\
\text { MG/L AS } \\
\text { CO3 } \\
(00452)\end{array}$ & $\begin{array}{l}\text { SULFATE } \\
\text { DIS- } \\
\text { SOLVED } \\
\text { (MG/I } \\
\text { AS SO4) } \\
(00945)\end{array}$ & $\begin{array}{l}\text { CHLO- } \\
\text { RIDE, } \\
\text { DIS- } \\
\text { SOLVED } \\
\text { (MG/L } \\
\text { AS CL) } \\
(00940)\end{array}$ & $\begin{array}{l}\text { FLUO- } \\
\text { RIDE, } \\
\text { DIS- } \\
\text { SOLVED } \\
\text { (MG/L } \\
\text { AS F) } \\
(00950)\end{array}$ & $\begin{array}{c}\text { SOLIDS, } \\
\text { RESIDUE } \\
\text { AT } 180 \\
\text { DEG. C } \\
\text { DIS- } \\
\text { SOLVED } \\
\text { (MG/L) } \\
(70300)\end{array}$ \\
\hline
\end{tabular}

\begin{tabular}{|c|c|c|c|c|c|c|c|c|c|c|c|c|}
\hline $\begin{array}{c}\text { OCT } 1991 \\
09 \ldots \\
\text { DEC }\end{array}$ & -- & -- & -- & -- & -- & -- & 115 & 0 & 7.5 & 51 & 0.10 & 206 \\
\hline${ }_{\operatorname{MAR}}^{17} \ddot{1992}$ & -- & -- & -- & -- & -- & -- & 91 & 0 & -- & -- & -- & -- \\
\hline$\underset{A P R}{04 \ldots}$ & 30 & 1.9 & 7.3 & 16 & 0.3 & 2.4 & 87 & 0 & 7.2 & 12 & $<.10$ & 119 \\
\hline$\underset{\text { JUN }}{08 \ldots}$ & -- & -- & -- & -- & -- & -- & 81 & 4 & -- & - & -- & -- \\
\hline JUL & 33 & 1.7 & 4.3 & 9 & .2 & 2.2 & 91 & 0 & 8.1 & 6.5 & $<.10$ & 116 \\
\hline AUG $16 \ldots$ & -- & -- & -- & -- & -- & -- & -- & -- & -- & -- & -- & -- \\
\hline $19 \ldots$ & 38 & 2.0 & 8.3 & 15 & .4 & 2.4 & 110 & 0 & 7.0 & 12 & $<.10$ & 133 \\
\hline
\end{tabular}




\section{ARKANSAS RIVER BASIN}

07198000 ILLINOIS RIVER NEAR GORE, OKLAHOMA--CONTINUED

WATER-QUALITY DATA, WATER YEAR OCTOBER 1991 TO SEPTEMBER 1992

\begin{tabular}{|c|c|c|c|c|c|c|c|c|c|c|c|}
\hline DATE & $\begin{array}{c}\text { SOLIDS, } \\
\text { SUM OF } \\
\text { CONSTI- } \\
\text { TUENTS, } \\
\text { DIS- } \\
\text { SOLVED } \\
(\text { MG/L) } \\
(70301)\end{array}$ & $\begin{array}{c}\text { SOLIDS, } \\
\text { DIS- } \\
\text { SOLVED } \\
\text { (TONS } \\
\text { PER } \\
\text { AC-FT) } \\
(70303)\end{array}$ & $\begin{array}{c}\text { SOLIDS, } \\
\text { DIS- } \\
\text { SOLVED } \\
\text { (TONS } \\
\text { PER } \\
\text { DAY) } \\
(70302)\end{array}$ & $\begin{array}{c}\text { NITRO- } \\
\text { GEN, } \\
\text { NO2+NO3 } \\
\text { DIS- } \\
\text { SOLVED } \\
\text { (MG/L } \\
\text { AS N) } \\
(00631)\end{array}$ & $\begin{array}{l}\text { NITRO- } \\
\text { GEN, } \\
\text { AMMONIA } \\
\text { DIS- } \\
\text { SOLVED } \\
\text { (MG/L } \\
\text { AS N) } \\
(00608)\end{array}$ & $\begin{array}{c}\text { NITRO- } \\
\text { GEN, } \\
\text { ORGANIC } \\
\text { TOTAI } \\
\text { (MG/I } \\
\text { AS N) } \\
(00605)\end{array}$ & $\begin{array}{l}\text { NITRO- } \\
\text { GEN, AM- } \\
\text { MONIA + } \\
\text { ORGANIC } \\
\text { TOTAL } \\
\text { (MG/L } \\
\text { AS N) } \\
(00625)\end{array}$ & $\begin{array}{c}\text { NITRO- } \\
\text { GEN, } \\
\text { TOTAL } \\
\text { (MG/L } \\
\text { AS N) } \\
(00600)\end{array}$ & $\begin{array}{c}\text { PHOS- } \\
\text { PHORUS } \\
\text { DIS- } \\
\text { SOLVED } \\
\text { (MG/L } \\
\text { AS P) } \\
(00666)\end{array}$ & $\begin{array}{c}\text { PHOS- } \\
\text { PHORUS } \\
\text { ORTHO } \\
\text { TOTAL } \\
\text { (MG/I } \\
\text { AS P) } \\
(70507)\end{array}$ & $\begin{array}{l}\text { PHOS- } \\
\text { PHORUS } \\
\text { ORTHO, } \\
\text { DIS- } \\
\text { SOLVED } \\
\text { (MG/L } \\
\text { AS P) } \\
(00671)\end{array}$ \\
\hline $\begin{array}{l}\mathrm{T} 1991 \\
09 \ldots \\
\mathrm{C}\end{array}$ & -- & -- & -- & 0.620 & 0.190 & 0.40 & 0.60 & 1.2 & 0.050 & 0.030 & 0.020 \\
\hline${ }_{R}^{17} \ddot{1992}$ & -- & -- & -- & 1.00 & .050 & .36 & .40 & 1.4 & .020 & .030 & .030 \\
\hline${ }_{\mathrm{R}}^{04} \cdots$ & 109 & 0.16 & 537 & 1.20 & $<.010$ & .27 & .30 & 1.5 & .020 & $<.010$ & $<.010$ \\
\hline $08 \ldots$ & -- & -- & -- & 1.30 & .020 & -- & $<.20$ & -- & .030 & $<.010$ & $<.010$ \\
\hline $03 \ldots$ & 106 & .16 & 1280 & 1.30 & .020 & .18 & .20 & 1.5 & .050 & .010 & $<.010$ \\
\hline $16 \ldots$ & -- & -- & -- & -- & -- & -- & -- & -- & -- & -- & -- \\
\hline $19 \ldots$ & 126 & .18 & 54.2 & .260 & .390 & .32 & .70 & .96 & .050 & .030 & .020 \\
\hline
\end{tabular}




\section{STREAMFLOW}

07245000 Canadian River near Whitefield, Oklahoma

LOCATION.--Lat $35^{\circ} 15^{\prime} 50^{\prime \prime}$, long 95 $14^{\prime} 21^{\prime \prime}$, in SE1/4SE1/4, sec.12, T.9 N., R.19 E., Haskell County, on left downstream bank at end of bridge, on State Highway 2, $0.8 \mathrm{mi}$ north of Whitefield, $5.5 \mathrm{mi}$ upstream from Taleka (Snake) Creek, 8.2 mi downstream from Eufaula Dam, and at mile 18.8.

DRAINAGE AREA.--47,576 $\mathrm{mi}^{2}$, of which $9,700 \mathrm{mi}^{2}$ is probably noncontributing.

AVERAGE DISCHARGE.--25 years (water years 1939-63), 6,005 $\mathrm{ft}^{3} / \mathrm{s} ; 25$ years (water years 1968-92), $6,477 \mathrm{ft}^{3} / \mathrm{s}$.

EXTREMES.--July 1938 to current year: Maximum discharge, $281,000 \mathrm{ft}^{3} / \mathrm{s}$ May 10, 1943; minimum daily, $0.4 \mathrm{ft}^{3} / \mathrm{s}$ Oct. $8,1956$.

REMARKS.--Records fair. Prior to February 1964, occasional slight regulation by Conchas Lake in New Mexico and except for $54 \mathrm{mi}^{2}$ of intervening area, completely regulated thereafter by Eufaula Lake. Satellite telemeter at station.

Monthly and yearly discharge

\begin{tabular}{lcccrr}
\hline Month & $\begin{array}{l}\text { Total } \\
\left(\mathrm{ft}^{3} / \mathrm{s}\right)\end{array}$ & $\begin{array}{c}\text { Maximum } \\
\text { daily } \\
\left(\mathrm{ft}^{3} / \mathrm{s}\right)\end{array}$ & $\begin{array}{c}\text { Minimum } \\
\text { daily } \\
\left(\mathrm{ft}^{3} / \mathrm{s}\right)\end{array}$ & $\begin{array}{r}\text { Mean } \\
\left(\mathrm{ft}^{3} / \mathrm{s}\right)\end{array}$ & $\begin{array}{r}\text { Runoff in } \\
\text { acre-feet }\end{array}$ \\
\hline October & 109,016 & 22,900 & 80 & 3,517 & 216,200 \\
November & 504,280 & 23,400 & 4,260 & 16,810 & $1,000,000$ \\
December & 629,340 & 43,200 & 3,270 & 20,300 & $1,248,000$ \\
January & 479,220 & 36,700 & 3,620 & 15,460 & 950,500 \\
February & 146,747 & 10,600 & 315 & 5,060 & 291,100 \\
March & 162,777 & 13,500 & 470 & 5,251 & 322,900 \\
April & 211,306 & 13,700 & 23 & 7,044 & 419,100 \\
May & 544,400 & 29,300 & 2,100 & 17,560 & $1,080,000$ \\
June & 804,020 & 39,000 & 9,320 & 26,800 & $1,595,000$ \\
July & 294,930 & 26,100 & 2,650 & 9,514 & 585,000 \\
August & 621,510 & 40,400 & 8,810 & 20,050 & $1,233,000$ \\
September & 417,240 & 22,300 & 8,340 & 13,910 & 827,600 \\
& & & & & $9,768,000$ \\
\hline Water Year 1992 & $4,924,786$ & 43,200 & 23 & 13,460 & \\
\hline
\end{tabular}




\section{STREAMFLOW}

07247000 Poteau River at Cauthron, Arkansas

LOCATION.--Lat $34^{\circ} 55^{\prime} 08^{\prime \prime}$, long $94^{\circ} 17^{\prime} 55^{\prime \prime}$, in NW1/4SW1/4, sec.16, T.3 N., R.31 W., Scott County, on right bank at downstream side of highway bridge at Cauthron, 2.9 mi downstream from Cross Creek, 7.8 mi downstream from Jones Creek, and at mile 109.0.

DRAINAGE AREA.--203 $\mathrm{mi}^{2}$.

AVERAGE DISCHARGE.--53 years, $224 \mathrm{ft}^{3} / \mathrm{s}$.

EXTREMES.--February 1939 to current year: Maximum discharge, 32,200 $\mathrm{ft}^{3} / \mathrm{s}$ May 20, 1960; no flow at times in most years.

REMARKS.--Records good. As of September 1974, flow from $92.2 \mathrm{mi}^{2}$ above this station is controlled by 16 floodwater-detention reservoirs with a total combined capacity of 39,082 acre-ft below the flood spillway crests, of which 33,524 acre-ft is flood-detention capacity, 2,100 acre-ft is water-supply storage, and 3,458 acre-ft is sediment-storage capacity.

Monthly and yearly discharge

\begin{tabular}{lccccr}
\hline Month & $\begin{array}{l}\text { Total } \\
\left(\mathrm{ft}^{3} / \mathrm{s}\right)\end{array}$ & $\begin{array}{c}\text { Maximum } \\
\text { daily } \\
\left(\mathrm{ft}^{3} / \mathrm{s}\right)\end{array}$ & $\begin{array}{c}\text { Minimum } \\
\text { daily } \\
\left(\mathrm{ft}^{3} / \mathrm{s}\right)\end{array}$ & $\begin{array}{c}\text { Mean } \\
\left(\mathrm{ft}^{3} / \mathrm{s}\right)\end{array}$ & $\begin{array}{c}\text { Runoff in } \\
\text { acre-feet }\end{array}$ \\
\hline October & 8,905 & 4,550 & 0.64 & 287 & 17,660 \\
November & 16,849 & 2,190 & 80 & 562 & 33,420 \\
December & 28,409 & 3,460 & 208 & 916 & 56,350 \\
January & 8,504 & 1,200 & 118 & 274 & 16,870 \\
February & 3,888 & 586 & 37 & 134 & 7,710 \\
March & 4,317 & 648 & 50 & 139 & 8,560 \\
April & 1,426 & 187 & 9.6 & 47.5 & 2,830 \\
May & 1,429 & 336 & 6.3 & 46.1 & 2,830 \\
June & 13,833 & 2,300 & 23 & 461 & 27,440 \\
July & 888 & 444 & .92 & 28.6 & 1,760 \\
August & 54 & 6.2 & .71 & 1.75 & 108 \\
September & 3,730 & 1,150 & 3.3 & 124 & 7,400 \\
& & & & & \\
\hline
\end{tabular}




\section{ARKANSAS RIVER BASIN \\ 07247000 POTEAU RIVER AT CAUTHRON, ARKANSAS}

PERIOD OF RECORD.--Water years 1945-61, 1975-79, December 1991-July 1992 (discontinued).

REMARKS.--Samples were collected on a six-week schedule and specific conductance, $\mathrm{pH}$, water temperature, alkalinity, and dissolved oxygen were determined in the field.

WATER-QUALITY DATA, WATER YEAR OCTOBER 1991 TO SEPTEMBER 1992

[Five-digit numbers in parentheses are STORET parameter codes used for computer storage of data; $\mathrm{K}$, non-ideal count; US/CM = microsiemens per centimeter at 25 degrees Celsius; $\mathrm{NTU}=$ nephelometric turbidity units; MG/L = milligrams per liter; $M$ M = millimeters; UM$\mathrm{MF}=$ micrometer membrane filter; $\mathrm{AC}-\mathrm{FT}=$ acre-feet, UG/L = micrograms per liter; $\mathrm{T} / \mathrm{DAY}$ $=$ tons per day]

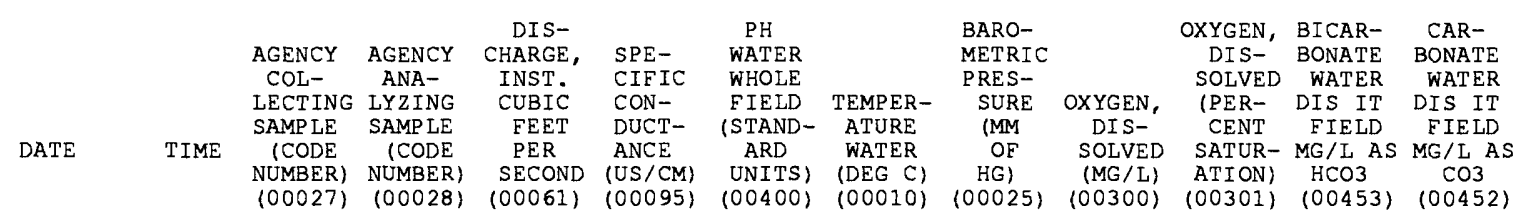

\begin{tabular}{|c|c|c|c|c|c|c|c|c|c|c|c|c|}
\hline $\begin{array}{c}\mathrm{DEC} 1991 \\
11 \ldots\end{array}$ & 0815 & 1028 & 80020 & 785 & 48 & 6.8 & 11.0 & -- & 10.2 & -- & 9 & 0 \\
\hline JAN 11992 & 0815 & -- & 80020 & 785 & -- & -- & & -- & -- & -- & -- & -- \\
\hline$\underset{M A R}{22 \ldots . .}$ & 0830 & 1028 & 80020 & 250 & 57 & 7.2 & 4.5 & 745 & 12.5 & 99 & 13 & 0 \\
\hline${ }_{A P R}^{10} \ldots$ & 1555 & 1028 & 80020 & 158 & 88 & 7.1 & 14.5 & 760 & 10.8 & 106 & 19 & 0 \\
\hline $\operatorname{MAY}^{13} \ldots$ & 1440 & 1028 & 80020 & 29 & 93 & 7.2 & 21.5 & 750 & 11.4 & 131 & 26 & 0 \\
\hline$\underset{\text { JUN }}{27 \ldots}$ & 0800 & 1028 & 80020 & 12 & 108 & 6.7 & 19.0 & 748 & 5.4 & 59 & 22 & 0 \\
\hline$\underset{\text { JUL }}{24 \ldots}$ & 1245 & 1028 & 80020 & 136 & 60 & 6.8 & 25.0 & 742 & 6.5 & 81 & 21 & 0 \\
\hline $\begin{array}{l}20 \ldots \\
20 \ldots \\
20 \ldots \\
20 \ldots \\
20 \ldots \\
20 \ldots\end{array}$ & $\begin{array}{l}1235 \\
1240 \\
1242 \\
1245 \\
1247 \\
1250\end{array}$ & $\begin{array}{l}1028 \\
1028 \\
1028 \\
1028 \\
1028 \\
1028\end{array}$ & $\begin{array}{l}1028 \\
1028 \\
1028 \\
1028 \\
1028 \\
1028\end{array}$ & $\begin{array}{l}3.0 \\
3.0 \\
3.0 \\
3.0 \\
3.0 \\
3.0\end{array}$ & $\begin{array}{l}106 \\
106 \\
107 \\
106 \\
106 \\
106\end{array}$ & $\begin{array}{l}7.1 \\
7.1 \\
7.1 \\
7.1 \\
7.1 \\
7.1\end{array}$ & $\begin{array}{l}27.5 \\
27.5 \\
27.5 \\
27.5 \\
28.0 \\
28.0\end{array}$ & $\begin{array}{l}750 \\
750 \\
750 \\
750 \\
750 \\
750\end{array}$ & $\begin{array}{l}8.0 \\
8.1 \\
8.0 \\
8.1 \\
8.0 \\
8.0\end{array}$ & $\begin{array}{l}103 \\
105 \\
103 \\
105 \\
104 \\
104\end{array}$ & $\begin{array}{l}-- \\
-- \\
-- \\
-- \\
-- \\
--\end{array}$ & $\begin{array}{l}-- \\
-- \\
-- \\
-- \\
-- \\
--\end{array}$ \\
\hline
\end{tabular}

\begin{tabular}{|c|c|c|c|c|c|c|c|c|c|c|}
\hline $\begin{array}{c}\text { NITRO- } \\
\text { GEN, } \\
\text { NO2+NO3 } \\
\text { DIS- } \\
\text { SOLVED } \\
\text { (MG/L } \\
\text { AS N) } \\
(00631)\end{array}$ & $\begin{array}{c}\text { NITRO- } \\
\text { GEN, } \\
\text { AMMONIA } \\
\text { DIS- } \\
\text { SOLVED } \\
\text { (MG/L } \\
\text { AS N) } \\
(00608)\end{array}$ & $\begin{array}{c}\text { NITRO- } \\
\text { GEN, } \\
\text { ORGANIC } \\
\text { TOTAL } \\
\text { (MG/L } \\
\text { AS N) } \\
(00605)\end{array}$ & $\begin{array}{c}\text { NITRO- } \\
\text { GEN, AM- } \\
\text { MONIA + } \\
\text { ORGANIC } \\
\text { TOTAL } \\
\text { (MG/L } \\
\text { AS N) } \\
(00625)\end{array}$ & $\begin{array}{l}\text { NITRO- } \\
\text { GEN, } \\
\text { TOTAL } \\
\text { (MG/L } \\
\text { AS N) } \\
(00600)\end{array}$ & $\begin{array}{c}\text { PHOS- } \\
\text { PHORUS } \\
\text { DIS- } \\
\text { SOLVED } \\
\text { (MG/L } \\
\text { AS P) } \\
(00666)\end{array}$ & $\begin{array}{c}\text { PHOS- } \\
\text { PHORUS } \\
\text { ORTHO } \\
\text { TOTAL } \\
\text { (MG/L } \\
\text { AS P) } \\
(70507)\end{array}$ & $\begin{array}{l}\text { PHOS- } \\
\text { PHORUS } \\
\text { ORTHO, } \\
\text { DIS- } \\
\text { SOLVED } \\
\text { (MG /L } \\
\text { AS P) } \\
(00671)\end{array}$ & $\begin{array}{l}\text { SEDI- } \\
\text { MENT, } \\
\text { SUS- } \\
\text { PENDED } \\
(\text { MG/L) } \\
(80154)\end{array}$ & $\begin{array}{c}\text { SEDI- } \\
\text { MENT, } \\
\text { DIS- } \\
\text { CHARGE, } \\
\text { SUS- } \\
\text { PENDED } \\
\text { (T/DAY) } \\
(80155)\end{array}$ & $\begin{array}{c}\text { SED. } \\
\text { SUSP. } \\
\text { SIEVE } \\
\text { DIAM. } \\
\text { F INER } \\
\text { THAN } \\
.062 \mathrm{MM} \\
(70331)\end{array}$ \\
\hline
\end{tabular}

\begin{tabular}{|c|c|c|c|c|c|c|c|c|c|c|c|}
\hline $\begin{array}{c}\text { DEC } 1991 \\
11 \text { JAN } 1992\end{array}$ & 0.290 & 0.020 & 0.36 & 0.40 & -- & 0.050 & 0.050 & 0.040 & 14 & 30 & -- \\
\hline$\underset{M A R}{22 \ldots}$ & .190 & .020 & .28 & .30 & 0.49 & .100 & .080 & .060 & 6 & 4.1 & -- \\
\hline${ }_{A P R}^{10} \ldots$ & .620 & .040 & .37 & .40 & 1.0 & .100 & .110 & .100 & 20 & 8.5 & 98 \\
\hline$\underset{M A Y}{13} \ldots$ & .650 & .010 & .28 & .30 & .96 & .160 & .150 & .120 & 18 & 1.4 & 86 \\
\hline $\mathrm{JUN}^{27} \ldots$ & .460 & .080 & .42 & .50 & .97 & .260 & .220 & .190 & 25 & .81 & -- \\
\hline${ }_{\text {JUL }}^{24} \ldots$ & .180 & .040 & .35 & .40 & .56 & .090 & .100 & .070 & 30 & 11 & -- \\
\hline $20 \ldots$ & -- & -- & -- & -- & -- & -- & -- & -- & -- & -- & -- \\
\hline
\end{tabular}




\section{ARKANSAS RIVER BASIN \\ 07247015 POTEAU RIVER AT LOVING, OKLAHOMA}

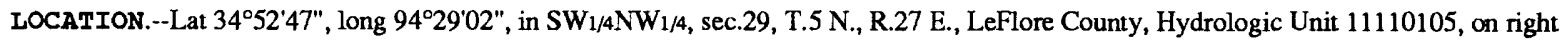
downstream bank of county road bridge, $0.6 \mathrm{mi}$ northwest of Loving, $1.0 \mathrm{mi}$ above Loving Creek, and at mile 93.6.

DRATNAGE AREA--269 $\mathrm{mi}^{2}$.

PERIOD OF RECORD.--Water years 1945-61, 1975-79, December 1991-July 1992 (discontinued).

REMARKS.--Samples were collected on a six-week schedule and specific conductance, $\mathrm{pH}$, water temperature, alkalinity, and dissolved oxygen were determined in the field.

\section{WATER-QUALITY DATA, WATER YEAR OCTOBER 1991 TO SEPTEMBER 1992}

[Five-digit numbers in parentheses are STORET parameter codes used for computer storage of data;K, non-ideal count; US/CM = microsiemens per centimeter at 25 degrees Celsius; $\mathrm{NTU}=$ nephelometric turbidity units; MG/L = milligrams per liter; MM = millimeters; UM$\mathrm{MF}=$ micrometer membrane filter; $\mathrm{AC}-\mathrm{FT}=$ acre-feet, $\mathrm{UG} / \mathrm{L}=$ micrograms per liter; $\mathrm{T} / \mathrm{DAY}$ $=$ tons per day]

\begin{tabular}{|c|c|c|c|c|c|c|c|c|c|c|c|c|}
\hline & & $\begin{array}{l}\text { AGENCY } \\
\text { COL- }\end{array}$ & $\begin{array}{l}\text { AGENCY } \\
\text { ANA- }\end{array}$ & $\begin{array}{l}\text { DIS- } \\
\text { CHARGE, } \\
\text { INST. }\end{array}$ & $\begin{array}{l}\text { SPE- } \\
\text { CIFIC }\end{array}$ & $\begin{array}{c}\text { PH } \\
\text { WATER } \\
\text { WHOLE }\end{array}$ & & $\begin{array}{l}\text { BARO- } \\
\text { METRIC } \\
\text { PRES- }\end{array}$ & & $\begin{array}{l}\text { OXYGEN } \\
\text { DIS- } \\
\text { SOLVED }\end{array}$ & $\begin{array}{c}\text { BICAR- } \\
\text { BONATE } \\
\text { WATER }\end{array}$ & $\begin{array}{r}\text { CAR- } \\
\text { BONATE } \\
\text { WATER }\end{array}$ \\
\hline & & LECTING & $\begin{array}{l}\text { LYZING } \\
\text { SAMPIF }\end{array}$ & CUBIC & CON- & $\begin{array}{l}\text { FIELD } \\
\text { STAND. }\end{array}$ & $\begin{array}{l}\text { TEMPER- } \\
\text { ATURF }\end{array}$ & SURE & OXYGEN, & (PER- & DIS IT & DIS IT \\
\hline$A T$ & [ME & $\begin{array}{c}\text { SAMPLE } \\
\text { (CODE } \\
\text { NUMBER) } \\
(00027)\end{array}$ & $\begin{array}{c}\text { SAMPLE } \\
\text { (CODE } \\
\text { NUMBER) } \\
(00028)\end{array}$ & $\begin{array}{l}\text { FEET } \\
\text { PER } \\
\text { SECOND } \\
(00061)\end{array}$ & $\begin{array}{l}\text { DUCI- } \\
\text { ANCE } \\
\text { (US/CM) } \\
\text { (00095) }\end{array}$ & $\begin{array}{l}\text { (SIAND- } \\
\text { ARD } \\
\text { UNITS) } \\
(00400)\end{array}$ & $\begin{array}{c}\text { ATURE } \\
\text { WATER } \\
\text { (DEG C) } \\
(00010)\end{array}$ & $\begin{array}{c}(\mathrm{MM} \\
\mathrm{OF} \\
\mathrm{HG}) \\
(00025)\end{array}$ & $\begin{array}{c}\text { SOLVED } \\
\text { (MG/L) } \\
(00300)\end{array}$ & $\begin{array}{c}\text { CENT } \\
\text { SATUR- } \\
\text { ATION) } \\
(00301)\end{array}$ & $\begin{array}{c}\text { MG/L AS } \\
\text { HCO3 } \\
(00453)\end{array}$ & $\begin{array}{c}\text { MG/L AS } \\
\mathrm{CO3} \\
(00452)\end{array}$ \\
\hline
\end{tabular}

\begin{tabular}{|c|c|c|c|c|c|c|c|c|c|c|c|}
\hline \multicolumn{12}{|l|}{ DEC 1991} \\
\hline $11 \ldots$ & 1015 & -- & 80020 & 838 & -- & -- & -- & -- & -- & - & -- \\
\hline JAN $\stackrel{11}{11} \ddot{9} 2$ & 1015 & 1028 & 80020 & 838 & 48 & 6.9 & 11.5 & 754 & 9.4 & 88 & 12 \\
\hline $\begin{array}{l}22 \ldots \\
\text { MAR }\end{array}$ & 1045 & 1028 & 80020 & 285 & 59 & 7.1 & 5.5 & 746 & 12.6 & 102 & 19 \\
\hline $11 \cdots$ & 0745 & 1028 & 80020 & 206 & 86 & 7.3 & 9.0 & 760 & 10.0 & 87 & 20 \\
\hline${ }_{\mathrm{MAY}}^{14} \ldots$ & 0735 & 1028 & 80020 & 36 & 93 & 7.0 & 19.5 & 750 & 7.4 & 82 & 21 \\
\hline $\begin{array}{l}27 \ldots \\
\text { JUN }\end{array}$ & 1000 & 1028 & 80020 & 50 & 115 & 7.0 & 18.5 & 750 & 6.0 & 65 & 27 \\
\hline$\underset{\text { JUL }}{24 \ldots}$ & 1445 & 1028 & 80020 & 260 & 65 & 6.8 & 28.0 & 742 & 6.5 & 86 & 17 \\
\hline $20 \ldots$ & $\begin{array}{l}1340 \\
1415\end{array}$ & 1028 & 80020 & 26 & 81 & 7.0 & 29.5 & 752 & 6.6 & 88 & -- \\
\hline $20 \ldots$ & $\begin{array}{l}1415 \\
1418\end{array}$ & 1028 & 1028 & 26 & 80 & 6.9 & 29.5 & 752 & 6.0 & 80 & -- \\
\hline & 1418 & 1028 & 1028 & 26 & 80 & 7.0 & 29.5 & 752 & 6.0 & 80 & -- \\
\hline & 1422 & 1028 & 1028 & 26 & 80 & 7.0 & 29.5 & 752 & 6.2 & 83 & -- \\
\hline $20 \ldots$ & 1425 & 1028 & 1028 & 26 & 81 & 7. & 29.5 & 752 & 6.2 & 83 & -- \\
\hline $20 \ldots$ & 1428 & 1028 & 1028 & 26 & 81 & 7.0 & 29.5 & 752 & 6.2 & 83 & -- \\
\hline & 1430 & 1028 & 1028 & 26 & 81 & 7.0 & 29.5 & 752 & 6.4 & 85 & -- \\
\hline $20 \ldots$ & 1433 & 1028 & 1028 & 26 & 81 & 7. & 29.5 & 752 & 6.4 & 85 & -- \\
\hline $20 \ldots$ & 1437 & 1028 & 1028 & 26 & 81 & 7. & 29.5 & 752 & 6.4 & 85 & -- \\
\hline & 1440 & 1028 & 1028 & 26 & 81 & 7. & 29.5 & 752 & 6. & 88 & -- \\
\hline & 1443 & 1028 & 1028 & 26 & 81 & 7. & 29.5 & 752 & 6.6 & 88 & -- \\
\hline 20. & 1446 & 1028 & 1028 & 26 & 81 & 7. & 29.5 & 752 & 6.6 & 88 & -- \\
\hline 20. & 1450 & 1028 & 1028 & 26 & 81 & 7. & 29.5 & 752 & 6.6 & 88 & -- \\
\hline $20 \ldots$ & 1453 & 1028 & 1028 & 26 & 81 & 7.0 & 29.5 & 752 & 6.4 & 85 & -- \\
\hline AUG & & & & & & & & & & & \\
\hline 25 . & 0755 & 1028 & 80020 & 2.9 & 114 & 7.0 & 24.5 & 754 & 4.8 & 58 & 29 \\
\hline DATE & $\begin{array}{c}\text { NITRO- } \\
\text { GEN, } \\
\text { NO2+NO3 } \\
\text { DIS- } \\
\text { SOLVED } \\
\text { (MG/L } \\
\text { AS N) } \\
(00631)\end{array}$ & $\begin{array}{c}\text { NITRO- } \\
\text { GEN, } \\
\text { AMMONIA } \\
\text { DIS- } \\
\text { SOLVED } \\
\text { (MG/L } \\
\text { AS N) } \\
(00608)\end{array}$ & $\begin{array}{l}\text { NITRO- } \\
\text { GEN, } \\
\text { ORGANIC } \\
\text { TOTAL } \\
\text { (MG/L } \\
\text { AS N) } \\
(00605)\end{array}$ & $\begin{array}{c}\text { NITRO- } \\
\text { GEN, AM- } \\
\text { MONIA + } \\
\text { ORGANIC } \\
\text { TOTAL } \\
\text { (MG/L } \\
\text { AS N) } \\
(00625)\end{array}$ & $\begin{array}{l}\text { NITRO- } \\
\text { GEN, } \\
\text { TOTAL } \\
\text { (MG/L) } \\
\text { AS N) } \\
(00600)\end{array}$ & $\begin{array}{c}\text { PHOS- } \\
\text { PHORUS } \\
\text { DIS- } \\
\text { SOLVED } \\
\text { (MG/L } \\
\text { AS P) } \\
(00666)\end{array}$ & $\begin{array}{c}\text { PHOS- } \\
\text { PHORUS } \\
\text { ORTHO } \\
\text { TOTAL } \\
\text { (MG/L } \\
\text { AS P) } \\
(70507)\end{array}$ & $\begin{array}{l}\text { PHOS- } \\
\text { PHORUS } \\
\text { ORTHO, } \\
\text { DIS- } \\
\text { SOLVED } \\
\text { (MG/L } \\
\text { AS P) } \\
(00671)\end{array}$ & $\begin{array}{l}\text { SEDI- } \\
\text { MENT, } \\
\text { SUS- } \\
\text { PENDED } \\
\text { (MG/L) } \\
(80154)\end{array}$ & $\begin{array}{c}\text { SEDI- } \\
\text { MENT, } \\
\text { DIS- } \\
\text { CHARGE, } \\
\text { SUS- } \\
\text { PENDED } \\
\text { (T/DAY) } \\
(80155)\end{array}$ & $\begin{array}{c}\text { SED. } \\
\text { SUSP. } \\
\text { SIEVE } \\
\text { DIAM. } \\
\text { FINER } \\
\text { THAN } \\
.062 \mathrm{MM} \\
(70331)\end{array}$ \\
\hline EC 1991 & & & & & & & & & & & \\
\hline JAN 11992 & 0.250 & 0.030 & 0.38 & 0.40 & 0.64 & 0.050 & 0.040 & 0.040 & 21 & 48 & -- \\
\hline $\begin{array}{l}22 \ldots \\
\operatorname{MAR}\end{array}$ & .160 & .020 & -- & .30 & .46 & .050 & .040 & 0.020 & 6 & 4.6 & - \\
\hline${ }_{P R}^{11} \cdots$ & .280 & .030 & .46 & .50 & -- & .070 & .060 & 0.030 & 12 & 6.7 & 94 \\
\hline $14 \ldots$ & .560 & .040 & .36 & .40 & .98 & .080 & .050 & 0.030 & 17 & 1.7 & 85 \\
\hline $\begin{array}{l}27 . \\
\text { JUN }\end{array}$ & .990 & .050 & .24 & .30 & 1.3 & .080 & .080 & 0.050 & 29 & 3.9 & -- \\
\hline $24 \ldots$ & .140 & .040 & .27 & .30 & .44 & .090 & .040 & 0.040 & 31 & 22 & -- \\
\hline $\begin{array}{l}20 . \\
\text { AUG }\end{array}$ & .065 & .040 & .46 & .50 & - & .070 & .040 & 0.030 & 14 & .98 & -- \\
\hline 25. & .086 & .050 & .54 & .60 & .68 & .020 & .050 & 0.030 & 8 & .06 & -- \\
\hline
\end{tabular}


ARKANSAS RIVER BASIN

\section{POTEAU RIVER AT HONTUBBY, OKLAHOMA}

LOCATION.--Lat 34'51'29", long 94³3'56", in SW1/4SE1/4, sec. 33, T.5 N., R.26 E., LeFlore County, Hydrologic Unit 11110105 , at county road bridge, $.6 \mathrm{mi}$ north of Hontubby.

DRATNAGE AREA --301 $\mathrm{mi}^{2}$.

PERIOD OF RECORD.--May 1992 (discontinued).

REMARKS.--Specific conductance, $\mathrm{pH}$, water temperature, alkalinity, and dissolved oxygen were determined in the field.

WATER-QUALITY DATA, HATER YEAR OCTOBER 1991 TO SEPTEMBER 1992

[Five-digit numbers in parentheses are STORET parameter codes used for computer storage of data; K, non-idela count; US/CM = microsiemens per centimeter at 25 degrees Celsius; $\mathrm{NTU}=$ nephelometric turbidity units; MG/L = milligrams per liter; MM = millimeters; UM$\mathrm{MF}=$ micrometer membrane filter; $\mathrm{AC}-\mathrm{FT}=$ acre-feet, UG/L = micrograms per liter; $\mathrm{T} / \mathrm{DAY}$ = tons per day]

\begin{tabular}{|c|c|c|c|c|c|c|c|c|c|c|c|}
\hline DATE & TIME & $\begin{array}{l}\text { AGENCY } \\
\text { COL- } \\
\text { IECTING } \\
\text { SAMPLE } \\
\text { (CODE } \\
\text { NUMBER) } \\
(00027 \text { ) }\end{array}$ & $\begin{array}{l}\text { AGENCY } \\
\text { ANA- } \\
\text { LYZING } \\
\text { SAMPLE } \\
\text { (CODE } \\
\text { NUMBER) } \\
(00028)\end{array}$ & $\begin{array}{c}\text { DIS- } \\
\text { CHARGE, } \\
\text { INST. } \\
\text { CUBIC } \\
\text { FEET } \\
\text { PER } \\
\text { SECOND } \\
(00061)\end{array}$ & $\begin{array}{l}\text { SPE- } \\
\text { CIFIC } \\
\text { CON- } \\
\text { DUCT- } \\
\text { ANCE } \\
\text { (US/CM) } \\
(00095)\end{array}$ & $\begin{array}{c}\text { PH } \\
\text { WATER } \\
\text { WHOLE } \\
\text { FIELD } \\
\text { (STAND- } \\
\text { ARD } \\
\text { UNITS) } \\
\text { (00400) }\end{array}$ & $\begin{array}{c}\text { TEMPER- } \\
\text { ATURE } \\
\text { WATER } \\
\text { (DEG C) } \\
(00010)\end{array}$ & $\begin{array}{l}\text { BARO- } \\
\text { METRIC } \\
\text { PRES- } \\
\text { SURE } \\
\text { (MM } \\
\text { OF } \\
\text { HG) } \\
(00025)\end{array}$ & $\begin{array}{c}\text { OXYGEN, } \\
\text { DIS- } \\
\text { SOLVED } \\
(M G / L) \\
(00300)\end{array}$ & $\begin{array}{c}\text { OXYGEN, } \\
\text { DIS- } \\
\text { SOLVED } \\
\text { (PER- } \\
\text { CENT } \\
\text { SATUR- } \\
\text { ATION) } \\
(00301 \text { ) }\end{array}$ & $\begin{array}{c}\text { BICAR- } \\
\text { BONATE } \\
\text { WATER } \\
\text { DIS IT } \\
\text { FIELD } \\
\text { MG/L AS } \\
\text { HCO3 } \\
(00453)\end{array}$ \\
\hline $\begin{array}{c}\text { IAY } 1992 \\
27 \ldots\end{array}$ & 1315 & 1028 & 80020 & 60 & 101 & 6.8 & 19.5 & 750 & 5.7 & 63 & 27 \\
\hline DATE & $\begin{array}{c}\text { CAR- } \\
\text { BONATE } \\
\text { WATER } \\
\text { DIS IT } \\
\text { FIELD } \\
\text { MG/L AS } \\
\text { CO3 } \\
(00452)\end{array}$ & $\begin{array}{c}\text { NITRO- } \\
\text { GEN, } \\
\text { NO2+NO3 } \\
\text { DIS- } \\
\text { SOLVED } \\
\text { (MG/L } \\
\text { AS N) } \\
(00631)\end{array}$ & $\begin{array}{c}\text { NITRO- } \\
\text { GEN, } \\
\text { AMMONIA } \\
\text { DIS- } \\
\text { SOLVED } \\
\text { (MG/L } \\
\text { AS N) } \\
(00608)\end{array}$ & $\begin{array}{c}\text { NITRO- } \\
\text { GEN, } \\
\text { ORGANIC } \\
\text { TOTAL } \\
\text { (MG/L } \\
\text { AS N) } \\
(00605)\end{array}$ & $\begin{array}{l}\text { NITRO- } \\
\text { GEN, AM- } \\
\text { MONIA + } \\
\text { ORGANIC } \\
\text { TOTAL } \\
\text { (MG/L } \\
\text { AS N) } \\
(00625)\end{array}$ & $\begin{array}{l}\text { NITRO- } \\
\text { GEN, } \\
\text { TOTAL } \\
\text { (MG/L } \\
\text { AS N) } \\
(00600)\end{array}$ & $\begin{array}{c}\text { PHOS- } \\
\text { PHORUS } \\
\text { DIS- } \\
\text { SOLVED } \\
\text { (MG/I } \\
\text { AS P) } \\
(00666)\end{array}$ & $\begin{array}{l}\text { PHOS- } \\
\text { PHORUS } \\
\text { ORTHO } \\
\text { TOTAL } \\
\text { (MG/L } \\
\text { AS P) } \\
(70507)\end{array}$ & $\begin{array}{l}\text { PHOS- } \\
\text { PHORUS } \\
\text { ORTHO, } \\
\text { DIS- } \\
\text { SOLVED } \\
\text { (MG/L } \\
\text { AS P) } \\
(00671)\end{array}$ & $\begin{array}{l}\text { SEDI- } \\
\text { MENT, } \\
\text { SUS- } \\
\text { PENDED } \\
(M G / L) \\
(80154)\end{array}$ & $\begin{array}{c}\text { SEDI- } \\
\text { MENT, } \\
\text { DIS- } \\
\text { CHARGE, } \\
\text { SUS- } \\
\text { PENDED } \\
\text { (T/DAY) } \\
(80155)\end{array}$ \\
\hline $\begin{array}{c}\text { MAY } 1992 \\
27 \ldots\end{array}$ & 0 & 0.620 & 0.060 & 0.34 & 0.40 & 1.0 & 0.080 & 0.040 & 0.020 & 29 & 4.7 \\
\hline
\end{tabular}




\section{ARKANSAS RIVER BASIN \\ 07247250 BLACK FORK BELOW BIG CREEK NEAR PAGE, OKLAHOMA}

LOCATION.--Lat 34'52'46", long 94³0'40", in NE1/4SW1/4, sec.31, T.4 N., R.27 E., LeFlore County, Hydrologic Unit 11110105, on downstream side of bridge pier of county road bridge, $2.2 \mathrm{mi}$ above Haw Creek, $5.0 \mathrm{mi}$ north of Page, and at mile 24.6.

DRATNAGE AREA. --74.4 $\mathrm{mi}^{2}$.

PERIOD OF RECORD. --December 1991 to September 1992.

REMARKS.--Samples were collected on a six-week schedule and specific conductance, $\mathrm{pH}$, water temperature, alkalinity, and dissolved oxygen were determined in the field.

WATER-QUALITY DATA, WATER YEAR OCTOBER 1991 TO SEPTEMBER 1992

[Five-digit numbers in parentheses are STORET parameter codes used for computer storage of data; K, non-ideal count; US/CM = microsiemens per centimeter at 25 degrees Celsius; $\mathrm{NTU}=$ nephelometric turbidity units; MG/L = milligrams per liter; $M M=\mathrm{millimeters;} \mathrm{UM-}$ $\mathrm{MF}=$ micrometer membrane filter; $\mathrm{AC}-\mathrm{FT}=$ acre-feet, $\mathrm{UG} / \mathrm{L}=$ micrograms per 1 iter; $\mathrm{T} / \mathrm{DAY}$ = tons per day]

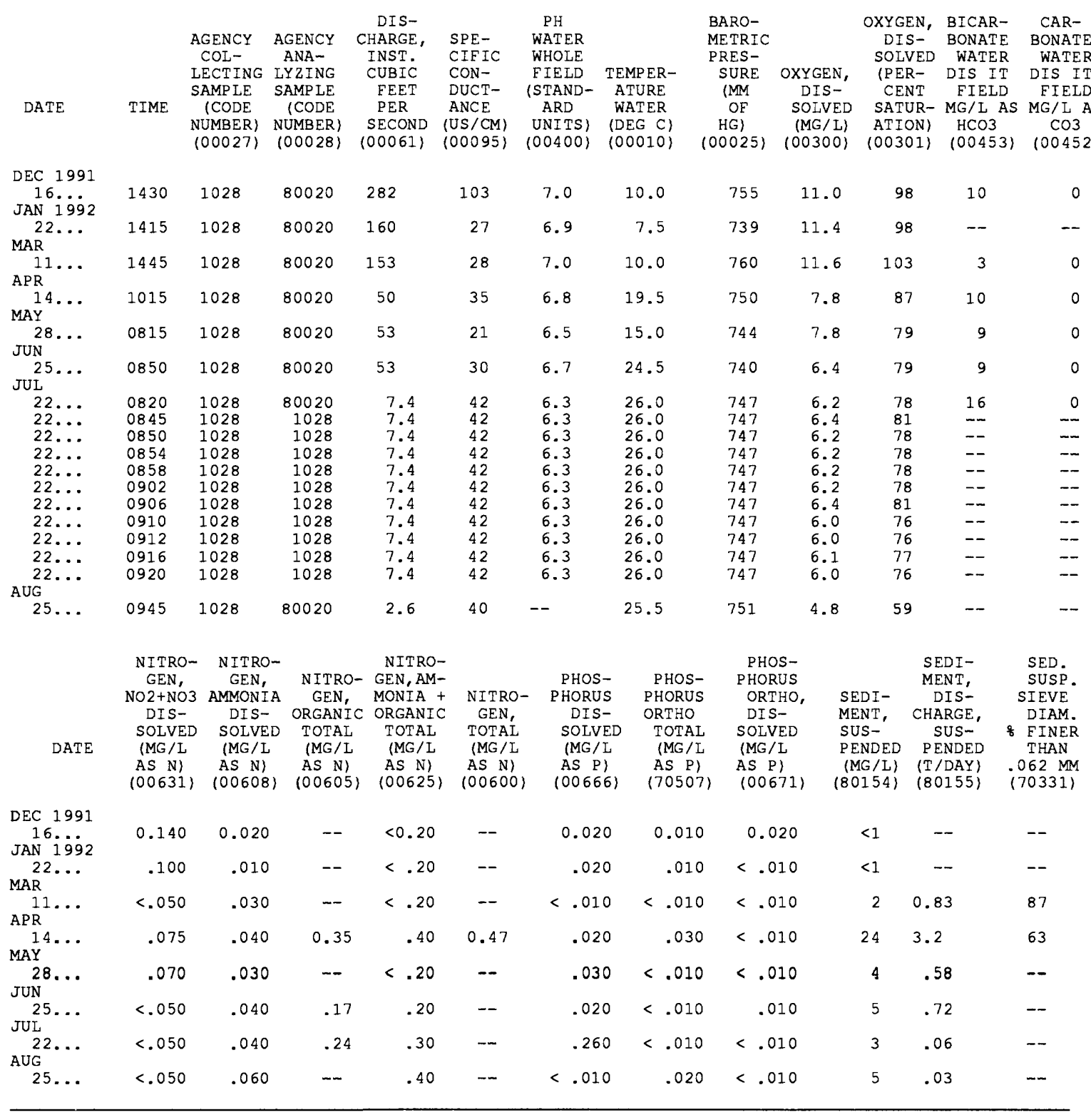




\section{ARKANSAS RIVER BASIN \\ 07247345 BLACK FORK AT HODGEN, OKLAHOMA}

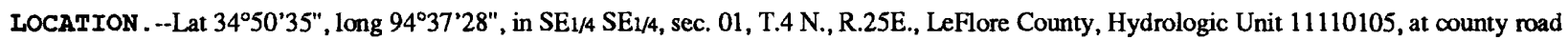
bridge .4 mi east of Hodgen, Oklahoma.

DRAINAGE AREA--179 $\mathrm{mi}^{2}$.

PERIOD OF RECORD.--December 1991 to September 1992.

REMARKS.--Samples were collected periodically, and specific conductance, $\mathrm{pH}$, water temperature, alkalinity, and dissolved oxygen were determined in the field.

WATER-QUALITY DATA, WATER YEAR OCTOBER 1991 TO SEPTEMBER 1992

[Five-digit numbers in parentheses are STORET parameter codes used for computer storage of data; K, non-ideal count; US/CM = microsiemens per centimeter at 25 degrees Celsius; NTU = nephelometric turbidity units; MG/L = milligrams per liter; MM = millimeters; UM$\mathrm{MF}=$ micrometer membrane filter; $\mathrm{AC}-\mathrm{FT}=$ acre-feet, UG/L = micrograms per liter; $\mathrm{T} / \mathrm{DAY}$ $=$ tons per day]

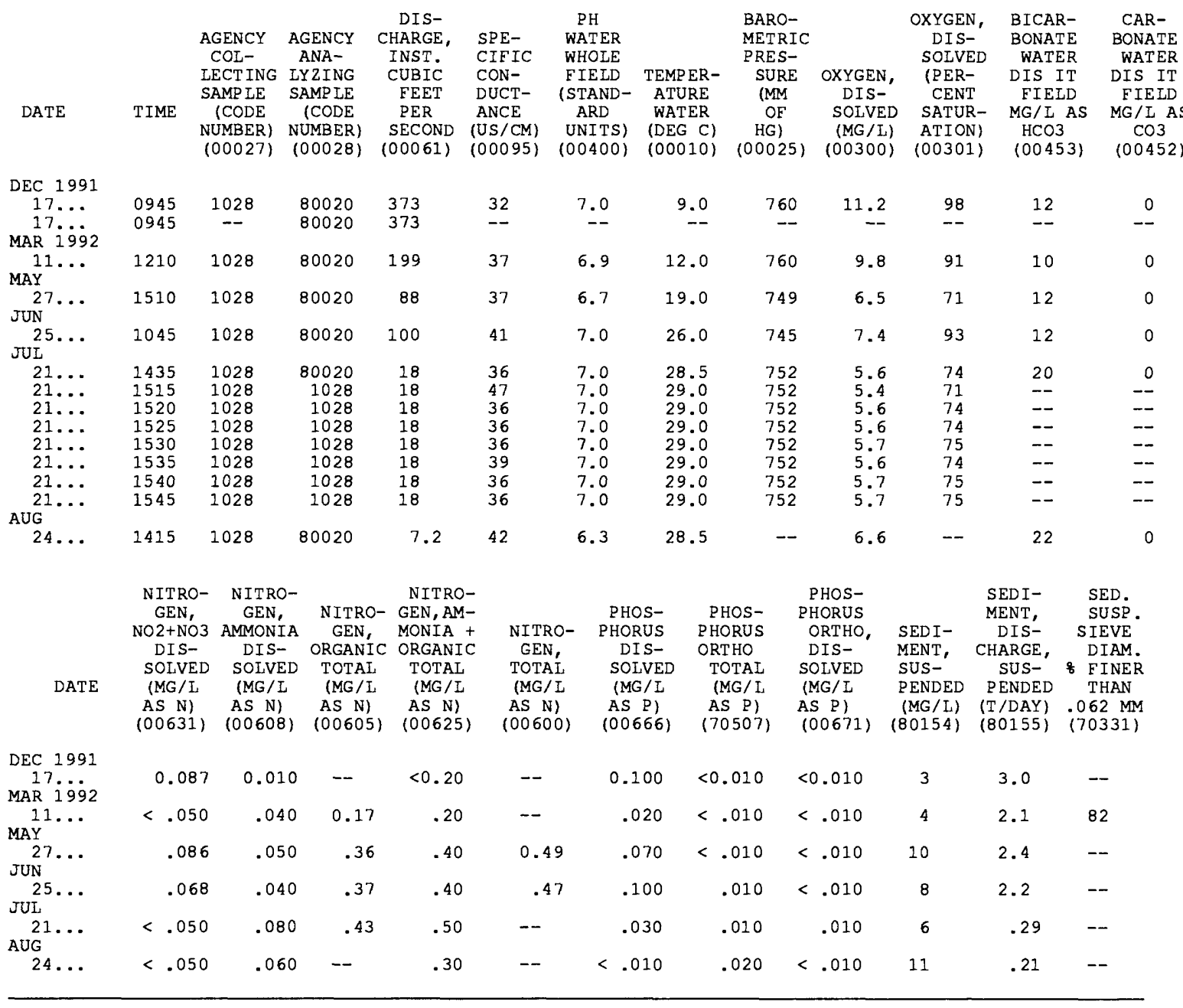




\section{ARKANSAS RIVER BASIN}

\section{FOURCHE MALINE NEAR LEFLORE, OKLAHOMA}

LOCATION.--Lat $34^{\circ} 55^{\prime} 11^{\prime \prime}$, long 9456'43", in NE1/4SE1/4, sec.11, T.5 N., R.22 E., LeFlore County, Hydrologic Unit 11110105 , at county road bridge $1.6 \mathrm{mi}$ east of LeFlore, Oklahoma.

DRAINAGE AREA. --270 $\mathrm{mi}^{2}$.

PERIOD OF RECORD.--December 1991 to September 1992.

REMARKS.--Samples were collected periodically, and specific conductance, $\mathrm{pH}$, water temperature, alkalinity, and dissolved oxygen were determined in the field.

\section{WATER-QUALITY DATA, WATER YEAR OCTOBER 1991 TO SEPTEMBER 1992}

[Five-digit numbers in parentheses are STORET parameter codes used for computer storage of data; K, non-ideal count; US/CM = microsiemens per centimeter at 25 degrees Celsius; $\mathrm{NTU}=$ nephelometric turbidity units; MG/L = miligrams per liter; MM = millimeters; UM$\mathrm{MF}=$ micrometer membrane filter; $\mathrm{AC}-\mathrm{FT}=$ acre-feet, $\mathrm{UG} / \mathrm{L}=\mathrm{micrograms}$ per liter; $\mathrm{I} / \mathrm{DAY}$ $=$ tons per day

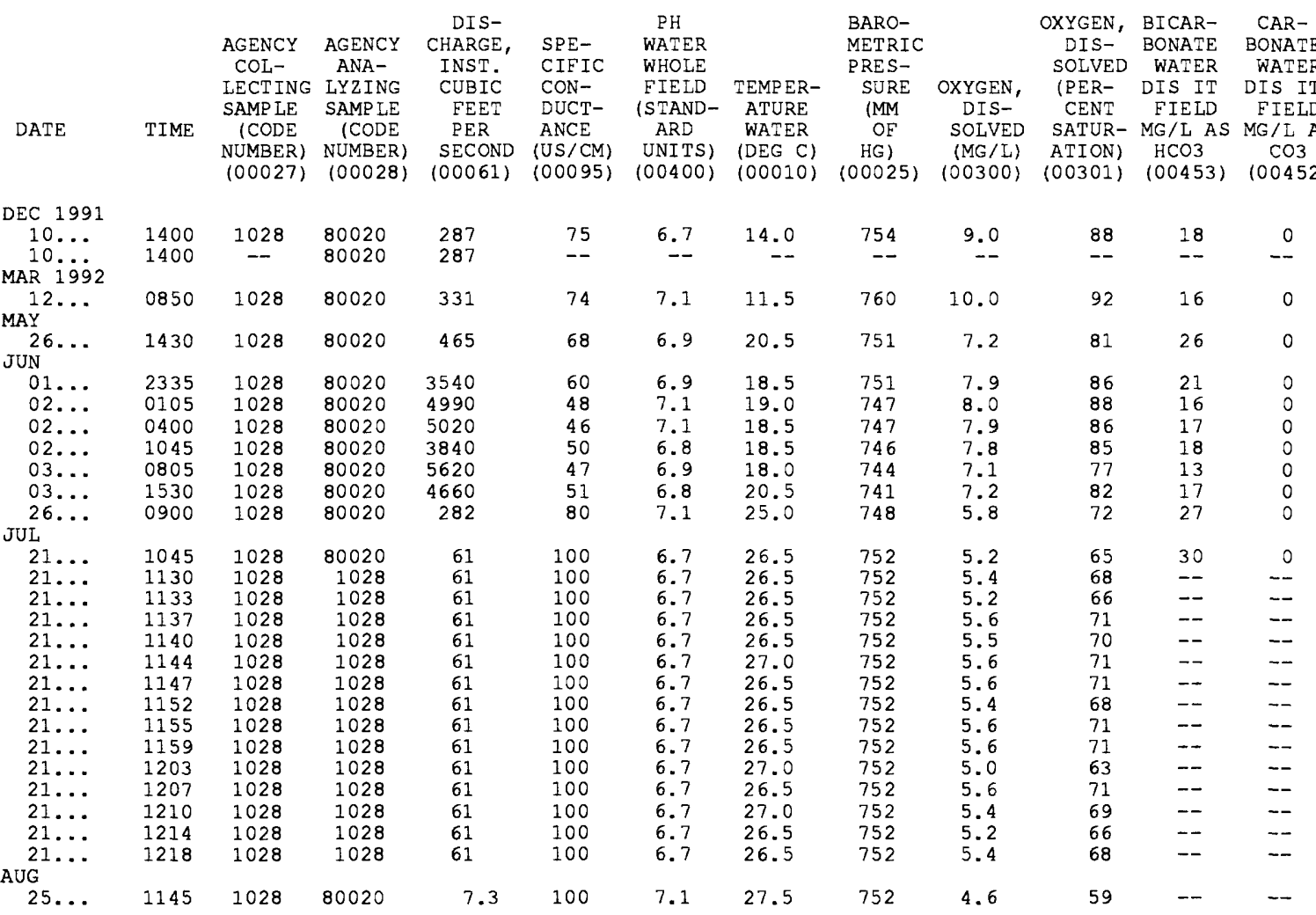


ARKANSAS RIVER BASIN

07247650 FOURCHE MALINE NEAR LEFLORE, OKLAHOMA--CONTINUED

WATER-QUALITY DATA, WATER YEAR OCTOBER 1991 TO SEPTEMBER 1992

\begin{tabular}{|c|c|c|c|c|c|c|c|c|c|c|c|}
\hline ATE & $\begin{array}{c}\text { NITRO- } \\
\text { GEN, } \\
\text { NO2+NO3 } \\
\text { DIS- } \\
\text { SOLVED } \\
\text { (MG/I } \\
\text { AS N) } \\
(00631)\end{array}$ & $\begin{array}{c}\text { NITRO- } \\
\text { GEN, } \\
\text { AMMONIA } \\
\text { DIS- } \\
\text { SOLVED } \\
\text { (MG/I } \\
\text { AS N) } \\
(00608)\end{array}$ & $\begin{array}{c}\text { NITRO- } \\
\text { GEN, } \\
\text { ORGANIC } \\
\text { TOTAL } \\
\text { (MG/I } \\
\text { AS N) } \\
(00605)\end{array}$ & $\begin{array}{l}\text { NITRO- } \\
\text { GEN, AM- } \\
\text { MONIA + } \\
\text { ORGANIC } \\
\text { TOTAL } \\
\text { (MG/L } \\
\text { AS N) } \\
(00625)\end{array}$ & $\begin{array}{c}\text { NITRO- } \\
\text { GEN, } \\
\text { TOTAL } \\
\text { (MG/L } \\
\text { AS N) } \\
(00600)\end{array}$ & $\begin{array}{c}\text { PHOS- } \\
\text { PHORUS } \\
\text { DIS- } \\
\text { SOIVED } \\
\text { (MG/L } \\
\text { AS P) } \\
(00666)\end{array}$ & $\begin{array}{c}\text { PHOS- } \\
\text { PHORUS } \\
\text { ORTHO } \\
\text { TOTAL } \\
\text { (MG/L } \\
\text { AS P) } \\
(70507)\end{array}$ & $\begin{array}{l}\text { PHOS- } \\
\text { PHORUS } \\
\text { ORTHO, } \\
\text { DIS- } \\
\text { SOLVED } \\
\text { (MG/L } \\
\text { AS P) } \\
(00671)\end{array}$ & $\begin{array}{l}\text { SEDI- } \\
\text { MENT, } \\
\text { SUS- } \\
\text { PENDED } \\
\text { (MG/L) } \\
\langle 80154\rangle\end{array}$ & $\begin{array}{l}\text { SEDI- } \\
\text { MENT, } \\
\text { DIS- } \\
\text { CHARGE, } \\
\text { SUS- } \\
\text { PENDED } \\
\text { (T/DAY) } \\
(80155)\end{array}$ & $\begin{array}{c}\text { SED. } \\
\text { SUSP. } \\
\text { SIEVE } \\
\text { DIAM. } \\
\text { \& FINER } \\
\text { THAN } \\
.062 \mathrm{MM} \\
(70331)\end{array}$ \\
\hline
\end{tabular}

\begin{tabular}{|c|c|c|c|c|c|c|c|c|c|c|c|}
\hline 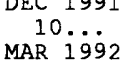 & 0.130 & 0.020 & 0.37 & 0.40 & 0.52 & 0.020 & 0.020 & 0.020 & 17 & 13 & -- \\
\hline $\begin{array}{l}12 \ldots \\
M A Y\end{array}$ & .056 & .040 & .45 & .50 & .56 & .100 & .100 & .050 & 49 & 44 & 98 \\
\hline $26 \ldots$ & .110 & .070 & .32 & .40 & .52 & .070 & .040 & .010 & 78 & 98 & -- \\
\hline $\begin{array}{l}01 \ldots \\
02 \ldots \\
02 \ldots \\
02 \ldots \\
03 \ldots \\
03 \ldots \\
26 \ldots \\
\text { JUL }\end{array}$ & $\begin{array}{r}.093 \\
.075 \\
.050 \\
.055 \\
<.050 \\
.050 \\
.170\end{array}$ & $\begin{array}{l}.040 \\
.040 \\
.040 \\
.060 \\
.020 \\
.060 \\
.040\end{array}$ & $\begin{array}{l}.56 \\
.56 \\
.56 \\
.52 \\
.47 \\
.53 \\
.35\end{array}$ & $\begin{array}{l}.60 \\
.60 \\
.60 \\
.60 \\
.50 \\
.60 \\
.40\end{array}$ & $\begin{array}{l}.71 \\
.69 \\
.66 \\
.66 \\
.57 \\
.66 \\
.57\end{array}$ & $\begin{array}{l}.070 \\
.110 \\
.060 \\
.060 \\
.100 \\
.060 \\
.140\end{array}$ & $\begin{array}{l}.040 \\
.070 \\
.030 \\
.090 \\
.030 \\
.070 \\
.060\end{array}$ & $\begin{array}{l}.040 \\
.060 \\
.030 \\
.020 \\
.020 \\
.020 \\
.020\end{array}$ & $\begin{array}{r}262 \\
333 \\
182 \\
94 \\
66 \\
75 \\
45\end{array}$ & $\begin{array}{r}2500 \\
4490 \\
2470 \\
975 \\
1000 \\
944 \\
34\end{array}$ & $\begin{array}{l}-- \\
-- \\
-- \\
-- \\
-- \\
-- \\
--\end{array}$ \\
\hline AUG $21 \ldots$ & .260 & .090 & .51 & .60 & .85 & .070 & .050 & .020 & 15 & 2.5 & -- \\
\hline $25 \ldots$ & -- & -- & .32 & .40 & .52 & -- & .040 & -- & 13 & .26 & -- \\
\hline
\end{tabular}




\section{ARKANSAS RIVER BASIN \\ 07247800 HOLSON CREEK AT SUMMERFIELD, OKLAHOMA}

IOCATION.--Lat 34'52'46", long 94'51'11", in SW1/4NW1/4, sec. 26, T.5 N., R.23 E., LeFlore County, Hydrologic Unit 11110105, at county road bridge, $1.4 \mathrm{mi}$ east of Summerfield, Oklahoma.

DRAINAGE AREA.--71.6 $\mathrm{mi}^{2}$.

PERIOD OF RECORD.--December 1991 to September 1992.

REMARKS.--Samples were collected periodically. Specific conductance, $\mathrm{pH}$, water temperature, alkalinity, and dissolved oxygen were determined in the field.

WATER-QUALITY DATA, WATER YEAR OCTOBER 1991 TO SEPTEMBER 1992

[Five-digit numbers in parentheses are STORET parameter codes used for computer storage of data; $\mathrm{K}$, ;non-ideal count; US/CM = microsiemens per centimeter at 25 degrees Celsius; $\mathrm{NTU}=$ nephelometric turbidity units; $M G / L=$ milligrams per liter; $M M$ = millimeters; UM$\mathrm{MF}=$ micrometer membrane filter; $\mathrm{AC}-\mathrm{FT}=$ acre-feet, UG/L = micrograms per liter; $\mathrm{T} / \mathrm{DAY}$ $=$ tons per day

\begin{tabular}{|c|c|c|c|c|c|c|c|c|c|c|}
\hline TIME & $\begin{array}{l}\text { AGENCY } \\
\text { COL- } \\
\text { LECTING } \\
\text { SAMPLE } \\
\text { (CODE } \\
\text { NUMBER) } \\
(00027)\end{array}$ & $\begin{array}{c}\text { AGENCY } \\
\text { ANA- } \\
\text { LYZING } \\
\text { SAMPLE } \\
\text { (CODE } \\
\text { NUMBER) } \\
(00028)\end{array}$ & $\begin{array}{c}\text { DIS- } \\
\text { CHARGE, } \\
\text { INST. } \\
\text { CUBIC } \\
\text { FEET } \\
\text { PER } \\
\text { SECOND } \\
(00061)\end{array}$ & $\begin{array}{l}\text { SPE- } \\
\text { CIFIC } \\
\text { CON- } \\
\text { DUCT- } \\
\text { ANCE } \\
\text { (US/CM) } \\
(00095)\end{array}$ & $\begin{array}{c}\text { PH } \\
\text { WATER } \\
\text { WHOLE } \\
\text { FIELD } \\
\text { (STAND- } \\
\text { ARD } \\
\text { UNITS) } \\
(00400)\end{array}$ & $\begin{array}{c}\text { TEMPER- } \\
\text { ATURE } \\
\text { WATER } \\
\text { (DEG C) } \\
(00010)\end{array}$ & $\begin{array}{l}\text { BARO- } \\
\text { METRIC } \\
\text { PRES- } \\
\text { SURE } \\
\text { (MM } \\
\text { OF } \\
\text { HG) } \\
(00025)\end{array}$ & $\begin{array}{c}\text { OXYGEN, } \\
\text { DIS- } \\
\text { SOIVED } \\
(M G / L) \\
(00300)\end{array}$ & $\begin{array}{c}\text { OXYGEN, } \\
\text { DIS- } \\
\text { SOLVED } \\
\text { (PER- } \\
\text { CENT } \\
\text { SATUR- } \\
\text { ATION) } \\
(00301 \text { ) }\end{array}$ & $\begin{array}{c}\text { BICAR- } \\
\text { BONATE } \\
\text { WATER } \\
\text { DIS IT } \\
\text { FIELD } \\
\text { MG/L AS } \\
\text { HCO3 } \\
(00453)\end{array}$ \\
\hline
\end{tabular}

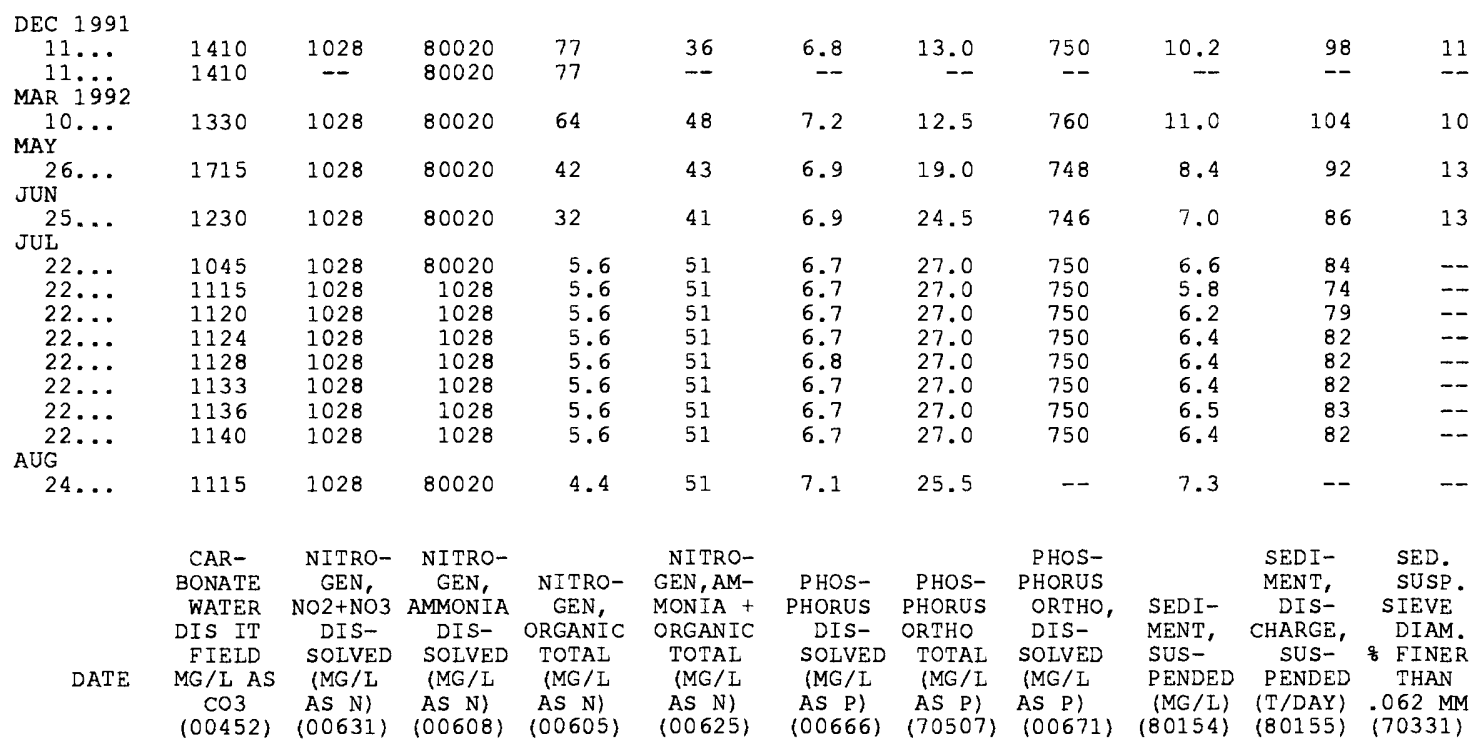

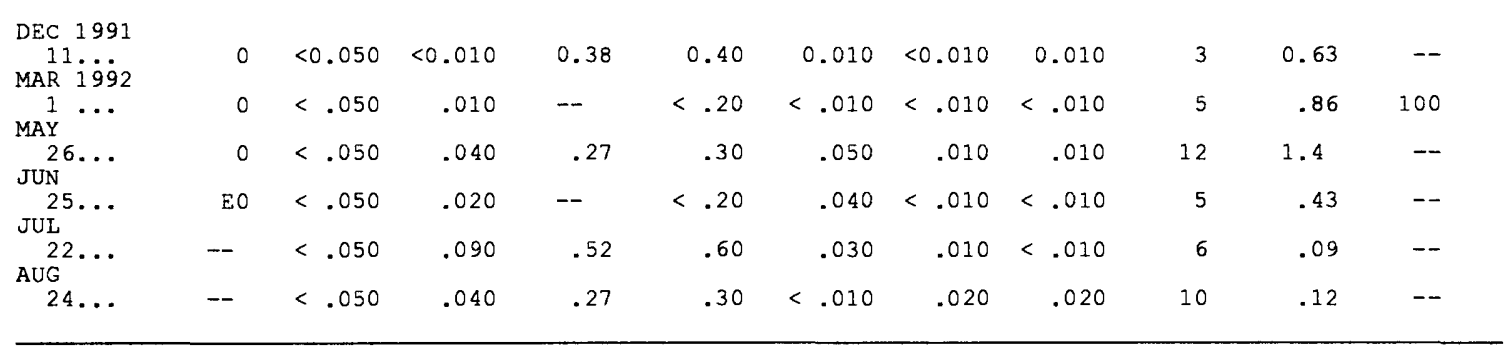




\section{STREAMFLOW}

07249400 James Fork near Hackett, Arkansas

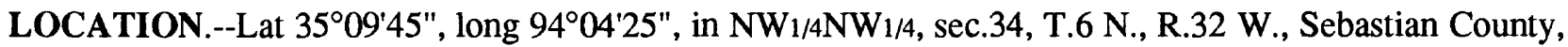
near left bank on downstream side of bridge on State Highway 45, $1.7 \mathrm{mi}$ south of Hackett, $2.0 \mathrm{mi}$ downstream from Elder Branch, $2.0 \mathrm{mi}$ upstream from small tributary, and $3.6 \mathrm{mi}$ upstream from Arkansas-Oklahoma State line.

DRAINAGE AREA.--147 $\mathrm{mi}^{2}$

AVERAGE DISCHARGE.--34 years, $141 \mathrm{ft}^{3} / \mathrm{s}$.

EXTREMES.--April 1958 to current year: Maximum discharge, 30,000 $\mathrm{ft}^{3} / \mathrm{s}$ May 14, 1968; no flow at times.

REMARKS.--Records good.

Monthly and yearly discharge

\begin{tabular}{lccccr}
\hline Month & $\begin{array}{c}\text { Total } \\
\left(\mathrm{ft}^{3} / \mathrm{s}\right)\end{array}$ & $\begin{array}{c}\text { Maximum } \\
\text { daily } \\
\left(\mathrm{ft}^{3} / \mathrm{s}\right)\end{array}$ & $\begin{array}{c}\text { Minimum } \\
\text { daily } \\
\left(\mathrm{ft}^{3} / \mathrm{s}\right)\end{array}$ & $\begin{array}{c}\text { Mean } \\
\left(\mathrm{ft}^{3} / \mathrm{s}\right)\end{array}$ & $\begin{array}{c}\text { Runoff in } \\
\text { acre-feet }\end{array}$ \\
\hline October & 14,960 & 6,480 & 3.0 & 483 & 29,670 \\
November & 11,448 & 2,030 & 73 & 382 & 22,710 \\
December & 16,683 & 2,990 & 111 & 538 & 33,090 \\
January & 4,650 & 655 & 70 & 150 & 9,220 \\
February & 2,105 & 150 & 37 & 72.6 & 4,180 \\
March & 2,180 & 153 & 41 & 70.3 & 4,320 \\
April & 1,539 & 353 & 14 & 51.3 & 3,050 \\
May & 1,104 & 181 & 12 & 35.6 & 2,190 \\
June & 4,839 & 1,050 & 35 & 161 & 9,600 \\
July & 1,449 & 601 & 14 & 46.7 & 2,870 \\
August & 342 & 16 & 8.2 & 11.0 & 678 \\
September & 1,891 & 672 & 9.8 & 63.0 & 3,750 \\
& & & & & 173 \\
\hline Water Year 1992 & 63,190 & 6,480 & 3.0 & & 125,300 \\
\hline
\end{tabular}




\section{STREAMFLOW}

07250000 Lee Creek near Van Buren, Arkansas

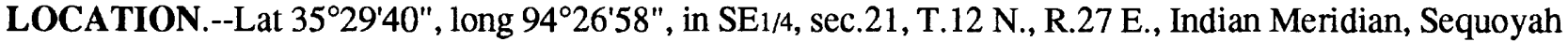
County, Okla., on right bank $300 \mathrm{ft}$ west of Arkansas-Oklahoma State line, $3.2 \mathrm{mi}$ downstream from Webbers Creek, $6.8 \mathrm{mi} \mathrm{no}^{2}$.

AVERAGE DISCHARGE.--48 years (1930-36, 1950-91), $521 \mathrm{ft}^{3} / \mathrm{s}$.

EXTREMES.--September 1930 to June 1937, October 1950 to current year: Maximum discharge, $80,600 \mathrm{ft}^{3} / \mathrm{s}$ May 6, 1960; no flow at times.

REMARKS.--Records good. Satellite telemeter at station.

Monthly and yearly discharge

\begin{tabular}{lccccc}
\hline & $\begin{array}{c}\text { Total } \\
\left(\mathrm{ft}^{3} / \mathrm{s}\right)\end{array}$ & $\begin{array}{c}\text { Maximum } \\
\text { daily } \\
\left(\mathrm{ft}^{3} / \mathrm{s}\right)\end{array}$ & $\begin{array}{c}\text { Minimum } \\
\text { daily } \\
\left(\mathrm{ft}^{3} / \mathrm{s}\right)\end{array}$ & $\begin{array}{c}\text { Mean } \\
\left(\mathrm{ft}^{3} / \mathrm{s}\right)\end{array}$ & $\begin{array}{c}\text { Runoff in } \\
\text { acre-feet }\end{array}$ \\
\hline October & 16,030 & 4,570 & 2.2 & 517 & 31,800 \\
November & 35,149 & 5,080 & 272 & 1,172 & 69,720 \\
December & 39,696 & 5,370 & 534 & 1,281 & 78,740 \\
January & 14,102 & 624 & 302 & 455 & 27,970 \\
February & 10,651 & 916 & 181 & 367 & 21,130 \\
March & 13,532 & 1,030 & 219 & 437 & 26,840 \\
April & 13,150 & 1,930 & 171 & 438 & 26,080 \\
May & 13,168 & 1,430 & 88 & 425 & 26,120 \\
June & 31,352 & 3,800 & 296 & 1,045 & 62,190 \\
July & 9,591 & 856 & 145 & 309 & 19,020 \\
August & 9,964 & 2,350 & 9.0 & 321 & 19,760 \\
September & 2,721 & 275 & 7.9 & 90.7 & 5,400 \\
& & & & & \\
\hline
\end{tabular}




\section{STREAMFLOW}

07250550 Arkansas River at James W. Trimble Lock and

Dam near Van Buren, Arkansas

LOCATION.--Lat 35²0'56", long 94¹7'54", in sec.28, T.8 N., R.31 W., Sebastian County, in James W. Trimble Lock and Dam control house on right bank, and at mile 308.9.

DRAINAGE AREA.-- $150,547 \mathrm{mi}^{2}$, of which $22,241 \mathrm{mi}^{2}$ is probably noncontributing.

AVERAGE DISCHARGE.-- 65 years, $32,750 \mathrm{ft}^{3} / \mathrm{s}$.

EXTREMES.--October 1927 to current year: Maximum discharge, 850,000 $\mathrm{ft}^{3} / \mathrm{s}$ May 12, 1943; no flow Nov. 2, 1975, Feb. 1, 1981, Oct. 17, 1987

REMARKS.--Records good. Prior to October 1969, published as 07250500 Arkansas River at Van Buren. Beginning Apr. 26, 1970, daily discharge computed from relation between discharge, head, and gate openings. Flow regulated by many locks, dams, and reservoirs upstream. Satellite telemeter at station.

Monthly and yearly discharge

\begin{tabular}{lrcccc}
\hline Month & $\begin{array}{c}\text { Total } \\
\left(\mathrm{ft}^{3} / \mathrm{s}\right)\end{array}$ & $\begin{array}{c}\text { Maximum } \\
\text { daily } \\
\left(\mathrm{ft}^{3} / \mathrm{s}\right)\end{array}$ & $\begin{array}{c}\text { Minimum } \\
\text { daily } \\
\left(\mathrm{ft}^{3} / \mathrm{s}\right)\end{array}$ & $\begin{array}{c}\text { Mean } \\
\left(\mathrm{ft}^{3} / \mathrm{s}\right)\end{array}$ & $\begin{array}{c}\text { Runoff in } \\
\text { acre-feet }\end{array}$ \\
\hline October & 483,135 & 93,400 & 135 & 15,580 & 958,300 \\
November & $1,359,600$ & 85,600 & 19,200 & 45,320 & $2,697,000$ \\
December & $1,963,900$ & 132,000 & 22,600 & 63,350 & $3,895,000$ \\
January & $1,210,600$ & 79,000 & 11,200 & 39,050 & $2,401,000$ \\
February & 699,000 & 42,600 & 1,250 & 24,100 & $1,386,000$ \\
March & 632,670 & 38,200 & 80 & 20,410 & $1,255,000$ \\
April & 876,201 & 59,200 & 31 & 29,210 & $1,738,000$ \\
May & $1,009,740$ & 56,900 & 3,390 & 32,570 & $2,003,000$ \\
June & $2,582,500$ & 117,000 & 25,700 & 86,080 & $5,122,000$ \\
July & $1,907,600$ & 79,300 & 45,400 & 61,540 & $3,784,000$ \\
August & $1,942,700$ & 108,000 & 26,300 & 62,670 & $3,853,000$ \\
September & $1,148,100$ & 63,300 & 12,700 & 38,270 & $2,277,000$ \\
& & & & & \\
\hline
\end{tabular}




\section{ARKANSAS RIVER BASIN}

07250550 ARKANSAS RIVER AT JAMES W. TRIMBLE LOCK AND DAM NEAR VAN BUREN, ARKANSAS

(National tritium station)

(National stream-quality accounting network station)

PERIOD OF RECORD.--Oct. 1969 to current water year.

PERIOD OF DAILY RECORD.--

SPECIFIC CONDUCTANCE: Oct. 1969 to Sept. 1981.

WATER TEMPERATURES: Oct. 1969 to Sept. 1972, Mar. 1974 to Sept. 1981.

SUSPENDED SEDIMENT DISCHARGE: Oct. 1970 to Sept. 1981.

INSTRUMENTATION.--Water-quality monitor Dec. 1969 to Sept. 1981.

REMARKS . --Samples were collected bimonthly and specific conductance, $\mathrm{pH}$, water temperature, dissolved oxygen, and alkalinity were determined in the field.

\section{WATER-QUALITY DATA, WATER YEAR OCTOBER 1991 TO SEPTEMBER 1992}

[Five-digit numbers in parentheses are STORET parameter codes used for computer storage of data; K, non-ideal count; US/CM = microsiemens per centimeter at 25 degrees Celsius; $\mathrm{NTU}=$ nephelometric turbidity units; MG/L = milligrams per liter; MM = millimeters; UM$\mathrm{MF}=$ micrometer membrane filter; $\mathrm{AC}-\mathrm{ET}=$ acre-feet, $\mathrm{UG} / \mathrm{L}=$ micrograms per liter; $\mathrm{T} / \mathrm{DAY}$ = tons per day]

\begin{tabular}{|c|c|c|c|c|c|c|c|c|c|c|c|c|}
\hline DATE & TIME & $\begin{array}{l}\text { AGENCY } \\
\text { COL- } \\
\text { LECTING } \\
\text { SAMPLE } \\
\text { (CODE } \\
\text { NUMBER) } \\
(00027)\end{array}$ & $\begin{array}{l}\text { AGENCY } \\
\text { ANA- } \\
\text { LYZING } \\
\text { SAMPLE } \\
\text { (CODE } \\
\text { NUMBER) } \\
(00028 \text { ) }\end{array}$ & $\begin{array}{l}\text { DIS- } \\
\text { CHARGE, } \\
\text { INST. } \\
\text { CUBIC } \\
\text { FEET } \\
\text { PER } \\
\text { SECOND } \\
(00061)\end{array}$ & $\begin{array}{l}\text { SPE- } \\
\text { CIFIC } \\
\text { CON- } \\
\text { DUCT- } \\
\text { ANCE } \\
\text { (US/CM) } \\
(00095)\end{array}$ & $\begin{array}{c}\text { PH } \\
\text { (STAND- } \\
\text { ARD } \\
\text { UNITS) } \\
(00400)\end{array}$ & $\begin{array}{l}\text { TEMPER- } \\
\text { ATURE } \\
\text { WATER } \\
\text { (DEG C) } \\
(00010)\end{array}$ & $\begin{array}{c}\text { TUR- } \\
\text { BID- } \\
\text { ITY } \\
\text { (NTU) } \\
(00076)\end{array}$ & $\begin{array}{c}\text { O } \\
\text { OXYGEN, } \\
\text { DIS- } \\
\text { SOLVED } \\
\text { (MG/L) } \\
(00300)\end{array}$ & $\begin{array}{l}\text { OXYGEN, } \\
\text { DIS- } \\
\text { SOLVED } \\
\text { (PER- } \\
\text { CENT } \\
\text { SATUR- } \\
\text { ATION) 1 } \\
\text { (O0301) } 1\end{array}$ & $\begin{array}{l}\text { COLI- } \\
\text { FORM, } \\
\text { FECAL, } \\
0.7 \\
\text { UM-MF } \\
\text { (COLS.) } \\
100 \text { ML) } \\
(31625 \text { ) }\end{array}$ & $\begin{array}{c}\text { STREP- } \\
\text { TOCOCCI } \\
\text { FECAL, } \\
\text { KF AGAR } \\
\text { (COLS. } \\
\text { PER } \\
\text { I00 ML) } \\
(31673)\end{array}$ \\
\hline $\begin{array}{l}\mathrm{EC} \\
10 \ldots \\
\mathrm{AN}\end{array}$ & 0815 & 80513 & 80020 & 29300 & 490 & 7.7 & 9.5 & 21 & 10.4 & 91 & 230 & 170 \\
\hline $22 \ldots$ & 1020 & 80513 & 80020 & 24400 & 525 & 7.8 & 2.0 & 24 & 12.8 & 94 & 34 & 740 \\
\hline${ }_{\text {UN }} 11 \ldots$ & 0730 & 80513 & 80020 & 32000 & 530 & 7.9 & 12.0 & 13 & 9.6 & 89 & 44 & 38 \\
\hline $04 \ldots$ & 0800 & 80513 & 80020 & 115000 & 440 & 7.2 & 19.5 & 71 & 7.6 & 84 & K900 & 2100 \\
\hline $20 \ldots$ & 1130 & 80513 & 80020 & 52100 & 515 & 8.1 & 26.0 & 28 & 6.8 & 84 & 87 & 160 \\
\hline DATE & TIME & $\begin{array}{l}\text { HARD- } \\
\text { NESS } \\
\text { TOTAL } \\
\text { (MG/L } \\
\text { AS } \\
\text { CACO3) } \\
(00900)\end{array}$ & $\begin{array}{l}\text { HARD- } \\
\text { NESS } \\
\text { NONCARB } \\
\text { DISSOLV } \\
\text { FLD. AS } \\
\text { CACO3 } \\
(\mathrm{MG} / \mathrm{L}) \\
(00904)\end{array}$ & $\begin{array}{l} \\
\text { CALCIUM } \\
\text { DIS- } \\
\text { SOLVED } \\
\text { (MG/L } \\
\text { AS CA) } \\
(00915)\end{array}$ & $\begin{array}{c}\text { MAGNE- } \\
\text { SIUM, } \\
\text { DIS- } \\
\text { SOLVED } \\
\text { (MG/L } \\
\text { AS MG) } \\
(00925)\end{array}$ & $\begin{array}{l}\text { SODIUM, } \\
\text { DIS- } \\
\text { SOLVED } \\
\text { (MG/L } \\
\text { AS NA) } \\
(00930)\end{array}$ & $\begin{array}{l}\text { SODIUM } \\
\text { PERCENT } \\
(00932)\end{array}$ & $\begin{array}{c}\text { SODIUM } \\
\text { AD- } \\
\text { SORP- } \\
\text { TION } \\
\text { RATIO } \\
\{00931\}\end{array}$ & $\begin{array}{l}\text { POTAS- } \\
\text { SIUM, } \\
\text { DIS- } \\
\text { SOLVED } \\
\text { (MG/I } \\
\text { AS K) } \\
(00935)\end{array}$ & $\begin{array}{c}\text { ALKA- } \\
\text { LINITY } \\
\text { LAB } \\
\text { (MG/L } \\
\text { AS } \\
\text { CACO3) } \\
(90410)\end{array}$ & $\begin{array}{c}\text { ALKA- } \\
\text { LINITY } \\
\text { WAT DIS } \\
\text { TOT FET } \\
\text { FIELD } \\
\text { MG/L AS } \\
\text { CACO3 } \\
(00418)\end{array}$ & $\begin{array}{c}\text { CAR- } \\
\text { BONATE } \\
\text { WATER } \\
\text { DIS IT } \\
\text { FIELD } \\
\text { MG/I AS } \\
\text { CO3 } \\
(00452)\end{array}$ \\
\hline $\begin{array}{l}\text { EC } \\
10 \ldots \\
\text { AN }\end{array}$ & 0815 & 90 & 35 & 25 & 6.8 & 59 & 58 & 3 & 3.1 & 67 & 56 & 0 \\
\hline $22 \ldots$ & 1020 & 110 & 32 & 30 & 7.4 & 52 & 51 & 2 & 3.0 & 76 & 73 & 0 \\
\hline $11 \ldots$ & 0730 & 120 & 30 & 37 & 7.8 & 47 & 44 & 2 & 3.0 & 91 & 94 & 0 \\
\hline UG & 0800 & 110 & 25 & 28 & 8.5 & 55 & 52 & 2 & 2.7 & 78 & 80 & 0 \\
\hline $20 \ldots$ & 1130 & 120 & 42 & 35 & 8.7 & 55 & 48 & 2 & 4.1 & 95 & 82 & 0 \\
\hline DATE & TIME & $\begin{array}{l}\text { BICAR- } \\
\text { BONATE } \\
\text { WATER } \\
\text { DIS IT } \\
\text { FIELD } \\
\text { MG/L AS } \\
\text { HCO3 } \\
(00453)\end{array}$ & $\begin{array}{l}\text { ALKA- } \\
\text { LINITY } \\
\text { WAT DIS } \\
\text { TOT IT } \\
\text { FIELD } \\
\text { MG/L AS } \\
\text { CACO3 } \\
(39086)\end{array}$ & $\begin{array}{l}\text { SULFATE } \\
\text { DIS- } \\
\text { SOLVED } \\
\text { (MG/L } \\
\text { AS SO4) } \\
(00945)\end{array}$ & $\begin{array}{l}\text { CHLO- } \\
\text { RIDE, } \\
\text { DIS- } \\
\text { SOLVED } \\
\text { (MG/L } \\
\text { AS CL) } \\
(00940)\end{array}$ & $\begin{array}{l}\text { FLUO- } \\
\text { RIDE, } \\
\text { DIS- } \\
\text { SOLVED } \\
\text { (MG/L } \\
\text { AS F) } \\
(00950)\end{array}$ & $\begin{array}{l}\text { SILICA, } \\
\text { DIS- } \\
\text { SOLVED } \\
\text { (MG/L } \\
\text { AS } \\
\text { SIO2) } \\
(00955)\end{array}$ & $\begin{array}{c}\text { SOLIDS, } \\
\text { RESIDUE } \\
\text { AT } 180 \\
\text { DEG. C } \\
\text { DIS- } \\
\text { SOLVED } \\
(\mathrm{MG} / \mathrm{L}) \\
(70300)\end{array}$ & $\begin{array}{c}\text { SOLIDS, } \\
\text { SUM OF } \\
\text { CONSTI- } \\
\text { TUENTS, } \\
\text { DIS- } \\
\text { SOLVED } \\
(\mathrm{MG} / \mathrm{L}) \\
(70301)\end{array}$ & $\begin{array}{c}\text { SOLIDS, } \\
\text { DIS- } \\
\text { SOLVED } \\
\text { (TONS } \\
\text { PER } \\
\text { DAY) } \\
(70302)\end{array}$ & $\begin{array}{l}\text { SOLIDS, } \\
\text { DIS- } \\
\text { SOLVED } \\
\text { (TONS } \\
\text { PER } \\
\text { AC-FT) } \\
(70303)\end{array}$ & $\begin{array}{c}\text { NITRO- } \\
\text { GEN, } \\
\text { NITRATE } \\
\text { DIS- } \\
\text { SOLVED } \\
\text { (MG /L } \\
\text { AS N) } \\
\{00618\}\end{array}$ \\
\hline $\begin{array}{l}E C \\
10 \ldots \\
\text { AN }\end{array}$ & 0815 & 68 & 56 & 34 & 94 & 0.20 & 4.5 & 284 & 262 & 22500 & 0.39 & 0.370 \\
\hline $22 \ldots$ & 1020 & 90 & 74 & 37 & 78 & .20 & 5.6 & 234 & 260 & 15400 & .32 & .410 \\
\hline$\underset{\mathrm{UN}}{11} \ldots$ & 0730 & 116 & 95 & 43 & 70 & .10 & 1.9 & 262 & 268 & 22600 & .36 & -- \\
\hline $04 \ldots$ & 0800 & 98 & 80 & 41 & 84 & $<.10$ & 4.7 & 278 & 274 & 86300 & .38 & -- \\
\hline $20 \ldots$ & 1130 & 100 & 82 & 37 & 80 & .20 & 7.2 & 297 & 279 & 41800 & .40 & -- \\
\hline
\end{tabular}


ARKANSAS RIVER BASIN

07250550 ARKANSAS RIVER AT JAMES W. TRIMBLE LOCK AND DAM NEAR

VAN BUREN, ARKANSAS--CONTINUED

WATER QDALITY DATA, WATER YEAR OCTOBER 1991 TO SEPTEMBER 1992

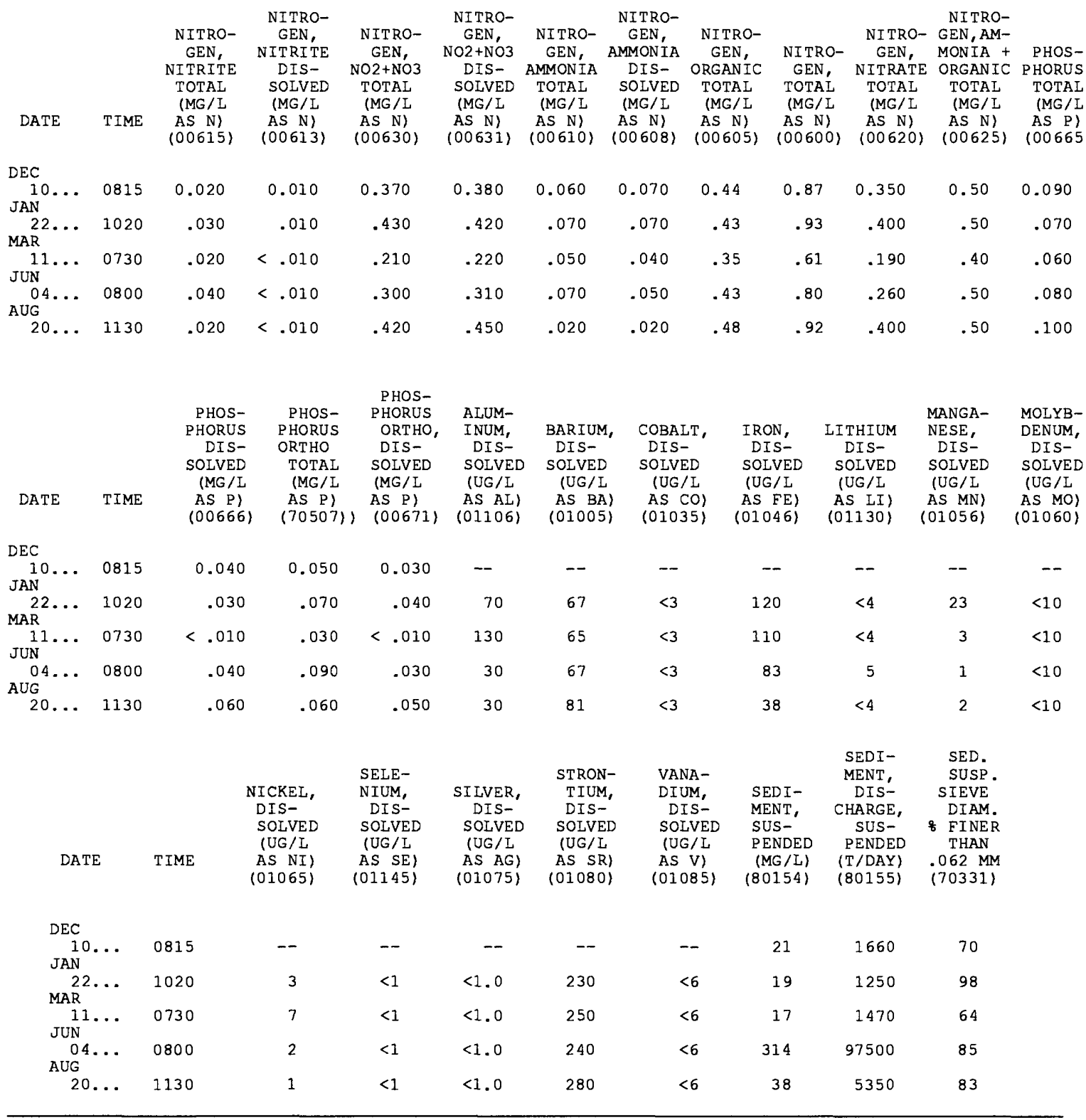

